\title{
PRELIMINARY KINETICS OF HIGH-TEMPERATURE REACTION OF CHLORINE AND STEAM \\ Yu-sung Yeh
}

M.S. Thesis Submitted to Iowa State University

Ames Laboratory, ERDA

Iowa State University

Ames, Iowa 50011

Date Transmitted: June 1976

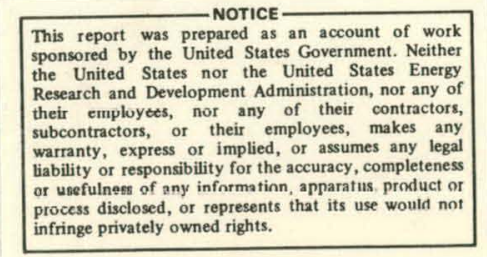

PREPARED FOR THE U. S. ENERGY RESEARCH AND DEVELOPMENT ADMINISTRATION UNDER CONTRACT NO. W-7405-eng-82. 


\section{DISCLAIMER}

This report was prepared as an account of work sponsored by an agency of the United States Government. Neither the United States Government nor any agency Thereof, nor any of their employees, makes any warranty, express or implied, or assumes any legal liability or responsibility for the accuracy, completeness, or usefulness of any information, apparatus, product, or process disclosed, or represents that its use would not infringe privately owned rights. Reference herein to any specific commercial product, process, or service by trade name, trademark, manufacturer, or otherwise does not necessarily constitute or imply its endorsement, recommendation, or favoring by the United States Government or any agency thereof. The views and opinions of authors expressed herein do not necessarily state or reflect those of the United States Government or any agency thereof. 


\section{DISCLAIMER}

Portions of this document may be illegible in electronic image products. Images are produced from the best available original document. 
This report was prepared as an account of work sponsored by the United States Government. Neither the United States nor the United States Energy Research and Development Administration, nor any of their employees, nor any of their contractors, subcontractors, or their employees, makes any warranty, express or implied, or assumes any legal liability or responsibility for the accuracy, completeness, or usefulness of any information, apparatis, product or process disclosed, or represents that its use would not inf ringe privately owned rights.

Available from: National Technical Information Service U. S. Department of Commerce P.O. Box 1553

Springfield, VA 22161

Price: Microfiche $\$ 2.25$ 
Preliminary kinetics of high-temperature reaction of chlorine and steam

by

Yu-sung Yeh

\author{
A Thesis Submitted to the \\ Graduate Faculty in Partial Fulfillment of \\ The Requirements for the Degree of \\ MASTER OF SCIENCE
}

Department: Chemical Engineering and Nuclear Engineering

Major: Chemical Engineering

Approved :

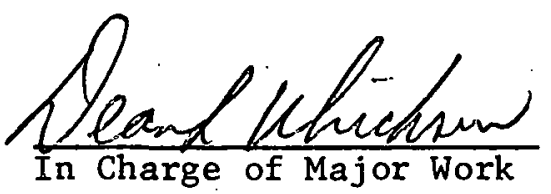

$\frac{\text { Alo. Durnet }}{\text { For the Major Department }}$

$\frac{\text { For the Graduate College }}{\text { Fondes }}$

Iowa State University

Ames, Iowa

1976 
TABLE OF CONTENTS

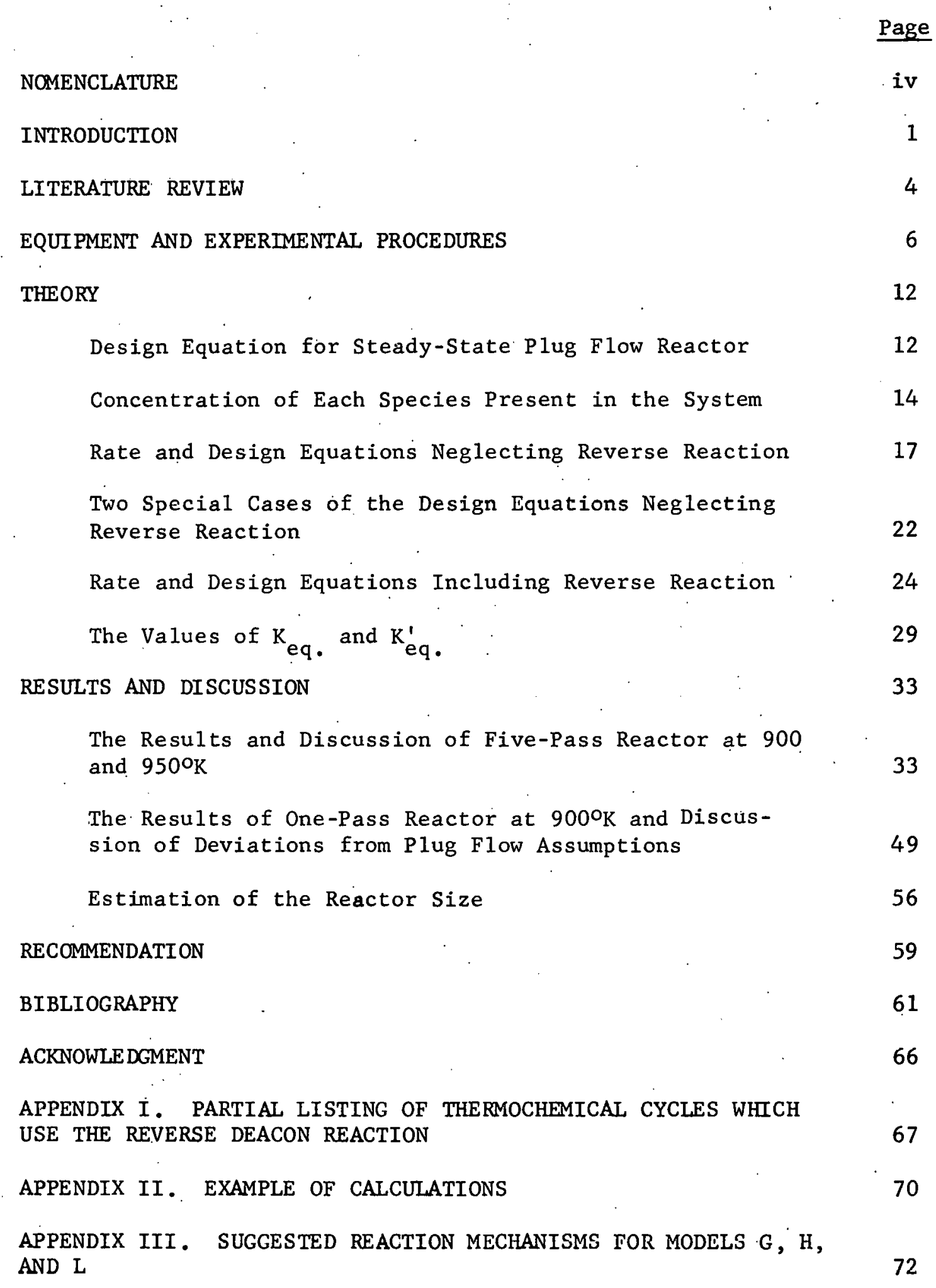


APPENDIX IV. CALCULATIONS OF FREQUENCY FACTOR AND ACTIVATION ENERGY FOR MODEL B

APPENDIX V. SUGGESTED REACTION MECHANISMS FOR MODEL B

APPENDIX VI. EXAMPLE OF CALCULATIONS OF REYNOLDS NUMBER

77 
NOMENCLATURE

${ }^{a}$

$\mathrm{C}_{\mathrm{A}}^{\mathrm{O}}$

$\mathrm{C}_{\mathrm{A}}$

$\mathrm{C}_{\mathrm{A}, \mathrm{eq}}$.

$\mathrm{F}_{\mathrm{A}}^{\mathrm{O}}$

$\mathrm{F}_{\mathrm{A}}$

$\mathrm{K}\left(\mathrm{K}^{\prime}\right)$

$\mathrm{K}_{\text {eq. }}\left(\mathrm{K}_{\text {eq. }}^{\prime}\right)$

A

$\mathrm{N}_{1}\left(\mathrm{~N}_{2}\right)$

$n_{t}$

$\mathrm{n}_{\mathrm{A}}$

$P$.

$\mathbf{R}$

$\mathrm{T}$

v

$\mathrm{U}$

$\mathrm{u}^{\circ}$

$\mathrm{X}_{\mathrm{Cl}_{2}}\left(\mathrm{x}_{\mathrm{H}_{2} \mathrm{O}}\right)$

$\mathrm{X}_{\mathrm{C} 1_{2}}^{*}\left(\mathrm{X}_{\mathrm{H}_{2} \mathrm{O}}^{*}\right)$ $\mathrm{X}_{\mathrm{Cl}_{2}}^{\mathrm{f}}\left(\mathrm{X}_{\mathrm{H}_{2} \mathrm{O}}^{\mathrm{f}}\right)$ activity of $A$ at equilibrium

initial concentration of $\mathrm{A}$ (g-moles/l)

concentration of $\mathrm{A}$ (g-moles/l)

concentration of $\mathrm{A}$ at equilibrium (g-moles/l)

molal feed rate of $A$ (g-moles/min.)

molal flow rate of $A$ (g-moles/min.)

equilibrium constant based on activity for Reaction I

(Reaction II)

equilibrium constant based on concentration for Reaction I

(Reaction II)

mole-fraction of $A$ at equilibrium

molal feed ratio of $\mathrm{H}_{2} \mathrm{O}$ to $\mathrm{Cl}_{2}\left(\mathrm{Cl}_{2}\right.$ to $\left.\mathrm{H}_{2} \mathrm{O}\right)$

total number of moles of the gaseous mixture at equilibrium number of moles of $A$ at equilibrium

pressure (atm.)

gas constant $\left(0.082 \mathrm{~atm} \cdot \ell / g-m o l e \cdot{ }^{\circ} \mathrm{K}\right)$

temperature $\left({ }^{\circ} \mathrm{K}\right)$

volume (l)

total volumetric flow rate $(\mathrm{cc} / \mathrm{min}$.)

initial total volumetric flow rate (cc/min.)

fraction of $\mathrm{Cl}_{2}\left(\mathrm{H}_{2} \mathrm{O}\right)$ converted into product when $\mathrm{Cl}_{2}\left(\mathrm{H}_{2} \mathrm{O}\right)$

is the limiting species

conversion based on $\mathrm{Cl}_{2}\left(\mathrm{H}_{2} \mathrm{O}\right)$ when $\mathrm{H}_{2} \mathrm{O}\left(\mathrm{Cl}_{2}\right)$ is limiting

final conversion of $\mathrm{Cl}_{2}\left(\mathrm{H}_{2} \mathrm{O}\right)$ when $\mathrm{Cl}_{2}\left(\mathrm{H}_{2} \mathrm{O}\right)$ is limiting 
${ }^{\epsilon} \mathrm{Cl}_{2}\left({ }^{\left(\mathrm{H}_{2} \mathrm{O}\right.}\right)$ fractional change in volume of the system between no conversion and complete conversion of $\mathrm{Cl}_{2}\left(\mathrm{H}_{2} \mathrm{O}\right)$

$\nu_{A} \quad$ activity coefficient of $A$ at equilibrium

subscript $A$ component identifier $\left(\mathrm{Cl}_{2}, \mathrm{HCl}, \mathrm{H}_{2} \mathrm{O}, \mathrm{O}_{2}\right)$ 


\section{ABSTRACT}

The kinetics of the reverse Deacon reaction, $\mathrm{Cl}_{2}+\mathrm{H}_{2} \mathrm{O}=2 \mathrm{HCl}+\frac{1}{2} \mathrm{O}_{2}$, at 900 and $950 \mathrm{~K}$ have been studied. A $6 \mathrm{~mm}$ ID five-pass tubular vycor reactor with a volume of $34 . c . c$. was used for most of the runs and the space-time for those runs ranged from 2 to 7 seconds: A $25 \mathrm{~mm}$. ID one-pass reactor with a volume of 172 c.c. was also used but it gave much less satisfactory results because of flow problems. The space-time in this case ranged from 19 to 75 seconds. Twelve rate models were tested for the forward reaction. Only two of them ( $\mathrm{k}_{1}\left(\mathrm{Cl}_{2}\right)$ and $\mathrm{k}_{\mathfrak{j}}\left(\mathrm{Cl}_{2}\right)^{2}\left(\mathrm{H}_{2} \mathrm{O}\right)$ ) gave reasonable correlation of the results. The values of $\mathrm{k}_{1}$ obtained are $0.032 \cdot \mathrm{sec}^{-1}$ at $900 \mathrm{~K}$ and $0.051 \mathrm{sec}^{-1}$ at $950 \mathrm{~K}$. The values of $\mathrm{k}$ are $980(g-\mathrm{moles} / \mathrm{l})^{-2} \mathrm{sec}^{-1}$ at $900 \mathrm{~K}$ and $1760(\mathrm{~g}-\mathrm{moles} / 1)^{-2} \mathrm{sec}^{-1}$ at $950 \mathrm{~K}$

- USERDA Report. IS-T-717. This work was performed under Contract W-7.405-eng-82 with the Energy Research and Development Administration. 


\section{INTRODUCTION}

Recently, people have begun to realize that the remaining energy reserves on this planet will become far too valuable as feedstocks for chemical production to be simply burned for their energy content. In the search for alternative energy sources, many scientists and engineers think hydrogen will be the answer. Hydrogen will be relatively cheap, abundant, and clean $(7,9,15,21,29,36,39,54,57)$.

Hydrogen is currently a major industrial chemical serving primarily as a chemical intermediate in the production of hydrogen-containing substances such as ammonia, methanol, and refined petroleum products.

There have been numerous papers published on the subject of the possible uses of hydrogen as a fuel. Hydrogen is particularly attractive for use in fuel cells'. It could also be used in any type of combustion engine, although some modification of conventional designs would be required (48). The major problems involved in using hydrogen as a common fuel are storage and transportation. Several scientists have researched this subject extensively $(8,21,28,36,53)$. Another major problem is that the securing of abundant supplies of pure hydrogen presents difficult technical problems. Most of the hydrogen produced currently in the United States and Europe is made by the steam reforming of natural gas or by the partial oxidation of oil. But the decreasing petroleum reserves in the next decade may make it necessary to adopt alternative methods of generation. Hydrogen can be formed by decomposing water. Direct thermal decomposition by heat 
occurs only at such high temperature that it is impractical to consider this as an engineering process (47).

The major candidate technologies for this century include electrolysis, new open-cycle thermochemical processes based on fossil fuel feedstocks $(44,50)$, and closed-cycle thermochemical generation methods (12). Longer range possibilities include biological and photolytic methods. Although electrolysis is the best developed of these technologies at present, cost estimates for hydrogen production by the above approaches indicate that electrolysis will probably not be the generally preferred method for large scale production unless off-peak electricity costs of under 5 mills per kilowatt-hour are available. Because pollution standards will become more strict and fossil fuel more expensive, the open-cycle processes seem to be less feasible for the future than the closed-cycle processes (54).

Several closed-cycle.thermochemical processes have been reported $(1,6,9,22,35)$. The processes, which ideally use water and heat and produce on ly hydrogen, oxygen, and degraded heat, involve the reactions of water with certain chemical compounds in a specified sequence at low temperatures. Ideally all the intermediates will be recycled within the system, but losses of recyclable species will still occur in a large-scale production process. That is going to cause some economic and environmental impact on the closed-cycle processes (10).

Computerized methods, used to sort out the thermodynamically sound reactions for the closed-cycle water-splitting processes, have also been devised (22). Up to now, most of the work has been done in 
understanding the basic chemistry, the thermodynamics and the processing characteristics.

The chemical reactions involved in the closed-cycle processes can be classified according to three essential functions: (a) water binding, (b) product recovery, and (c) reagent regeneration. A single reaction step can perform a dual function as in the reverse Deacon reaction, $\mathrm{Cl}_{2(\mathrm{~g})}+\mathrm{H}_{2} \mathrm{O}_{(\mathrm{g})} \rightleftharpoons 2 \mathrm{HCl}(\mathrm{g})+\frac{1}{2} \mathrm{O}_{2(\mathrm{~g})}$, in which the water binding reaction is combined with the oxygen recovery. That is why some of the reported processes have been built around the reverse Deacon reaction. For example, the iron chloride process, which was suggested by DeBeni (18),

$$
\begin{array}{ll}
\mathrm{H}_{2} \mathrm{O}+\mathrm{Cl}_{2} \longrightarrow 2 \mathrm{HCl}+\frac{1}{2} \mathrm{O}_{2} & \text { at } 973 \mathrm{O}^{\mathrm{K}} \\
2 \mathrm{HCl}+2 \mathrm{FeCl}_{2} \longrightarrow 2 \mathrm{FeCl}_{3}+\mathrm{H}_{2} & \text { at } 873^{\circ} \mathrm{K} \\
2 \mathrm{FeCl}_{3} \longrightarrow 2 \mathrm{FeCl}_{2}+\mathrm{Cl}_{2} & \text { at } 623^{\circ} \mathrm{K} \\
\text { net reaction } \mathrm{H}_{2} \mathrm{O} \longrightarrow \mathrm{H}_{2}+\frac{1}{2} \mathrm{O}_{2} &
\end{array}
$$

Others are shown in Appendix A.

The equilibrium conversion of the reverse Deacon reaction is $50.5 \%$ at $900^{\circ} \mathrm{K}$ and $55 \%$ at $950^{\circ} \mathrm{K}$ for equal-molal feed. A1though the thermodynamics of this reaction are weil known, the kinetics have not been studied thoroughly. The purpose of this study is to determine the reaction kinetics necessary for a comparative evaluation of the closedcycle thermochemical water-splitting processes. 
LITERATURE REVIEW

The oxidation of hydrochloric acid, which is called the Deacon reaction, has been of commercial interest since 1868 for manufacture. of chlorine $(38,49)$. The Deacon reaction, which was the first large scale gas-phase catalytic process used by the chemical industry, enjoyed wide usage in Europe but was never commercially practiced in the U.S.A. There have been a lot of patents issued regarding the catalysts and the operating conditions for the Deacon reaction (16).

Falckenstein (20) discussed the equilibrium constants for both the Deacon reaction and the reverse Deacon reaction. Arnold and Kobe (2) wrote the following equation for calculating the equilibrium constant of $4 \mathrm{HCl}+\mathrm{O}_{2} \rightleftharpoons 2 \mathrm{Cl}_{2}+2 \mathrm{H}_{2} \mathrm{O}$ :

$$
\begin{aligned}
\log \mathrm{K} & =\frac{5881.7}{\mathrm{~T}}-0.93035 \log \mathrm{T}+\left(1.3704 \times 10^{-5}\right) \mathrm{T} \\
& -\left(1.7581 \times 10^{-8}\right) \mathrm{T}^{2}-4.1744
\end{aligned}
$$

Johnstone (33) also published the free energy data for the Deacon reaction.

Jones (34) wrote his Ph.D. dissertation on the kinetics of the oxidation of hydrogen chloride. The three temperatures he used were 598, 613 , and $628^{\circ} \mathrm{K}$. He also studied the reverse Deacon reaction at 628 and $643^{\circ} \mathrm{K}$. His experiments were based on the heterogeneous catalytic reaction.

Few papers, most of them published before 1940, discussed the formation of hydrochloric acid (or hydrogen chloride) via the reverse Deacon reaction. Hirschkind (31) studied the reaction and gave a 
history of the attempts to utilize this reaction. He also stated that in order to make the reaction commercially practicable, a reducing agent such as carbon must be used to combine with the oxygen liberated by the reaction. Other authors also made the same argument $(14,26,32$, $41,43)$.

Barstow and Heath (4) have a patent for the equi-molal proportions of chlorine and steam reacted at $1000-1600^{\circ} \mathrm{C}$ in the substantial absence of reducing substances. Apparatus and various details of operation were described. Reference (11) also discusses running the reaction without a reducing agent. Ohkawa (40) has a patent to use ultraviolet light to make the dissolved chlorine react with the water to form hydrochloric acid.

Shelud'ko (51) used a catalyst containing $20 \%$ of $\mathrm{MgO}, 25 \%$ of $\mathrm{MgCl}_{2}$, and $25 \%$ of $\mathrm{CaO}$ to obtain a $97 \%$ yield of hydrogen chloride for reverse Deacon reaction, at $900^{\circ} \mathrm{C}$.

Euratom scientists have proposed a pilot plant reactor design to study the kinetics of the reverse Deacon reaction. Similar reactors have also been described by Musso in (19) and by Funk and Reinstrom (23) The proposed apparatus includes reaction and analytical sections.

The reaction section can be divided into the preheaters, the mixing chamber, and the reactor. Concentrated solutions of potassium iodide are used to trap the unreacted chlorine. Oxygen is determined by a gas chromatograph.

No one has obtained data which indicate either an empirical rate equation or a satisfactory mechanism. That information is needed for the calculation of the reactor size. 
EQUI PMENT AND EXPERIMENTAL PROCEDURES

Figure 1 illustrates the equipment setup used to study the chlorine reaction with steam. The chlorine gas cylinder was purchased from Matheson Gas Company and fitted with Matheson's mode1 B-15 regulator. Penntube's 1/4" flexible corrugated Teflon tube was used to connect the chlorine cylinder, the rotameter and the chlorine preheater. The rotameter, specially designed to handle chlorine, was purchased from Emerson Electric Co. The flow rate range is from 8 to 130 standard $\mathrm{cc} / \mathrm{min}$. Figure 2 illustrates the steam generator. The generator was made of stainless steel and was wrapped with a $1^{\prime \prime} \times 72^{\prime \prime}$ heavily insulated fibrous glass flexible electric heating tape. The heat input to the steam generator was controlled by a power-stat. A 30\# pressure gauge indicated the pressure in the steam generator. The control of the steam flow rates was achieved by using a 8513A Brooks' NRS needle valve with. a numerical handle. Pope's $9 \mathrm{~mm}$ ID Flo-Tite heat shrinkable tube was used to connect the steam generator and the steam preheater. Another heating tape, controlled by a temperature controller, was wrapped along the line from steam valve to steam preheater to prevent steam from condensing. The temperature of the heating tape was controlled around $180^{\circ} \mathrm{C}$.

The preheaters, reactor and water scrubber were made of Vycor tube. Figure 3 illustrates their size and arrangement. Two kinds of reactor were used. One was a five-pass $34 \mathrm{cc}$ reactor as shown in Figure 3. The other was a $25 \mathrm{~mm}$ ID $35 \mathrm{~cm}$ long one-pass $172 \mathrm{cc}$ reactor. The preheaters were put in two 7" long 1-1/4" ID Clam-she11 furnaces. The 


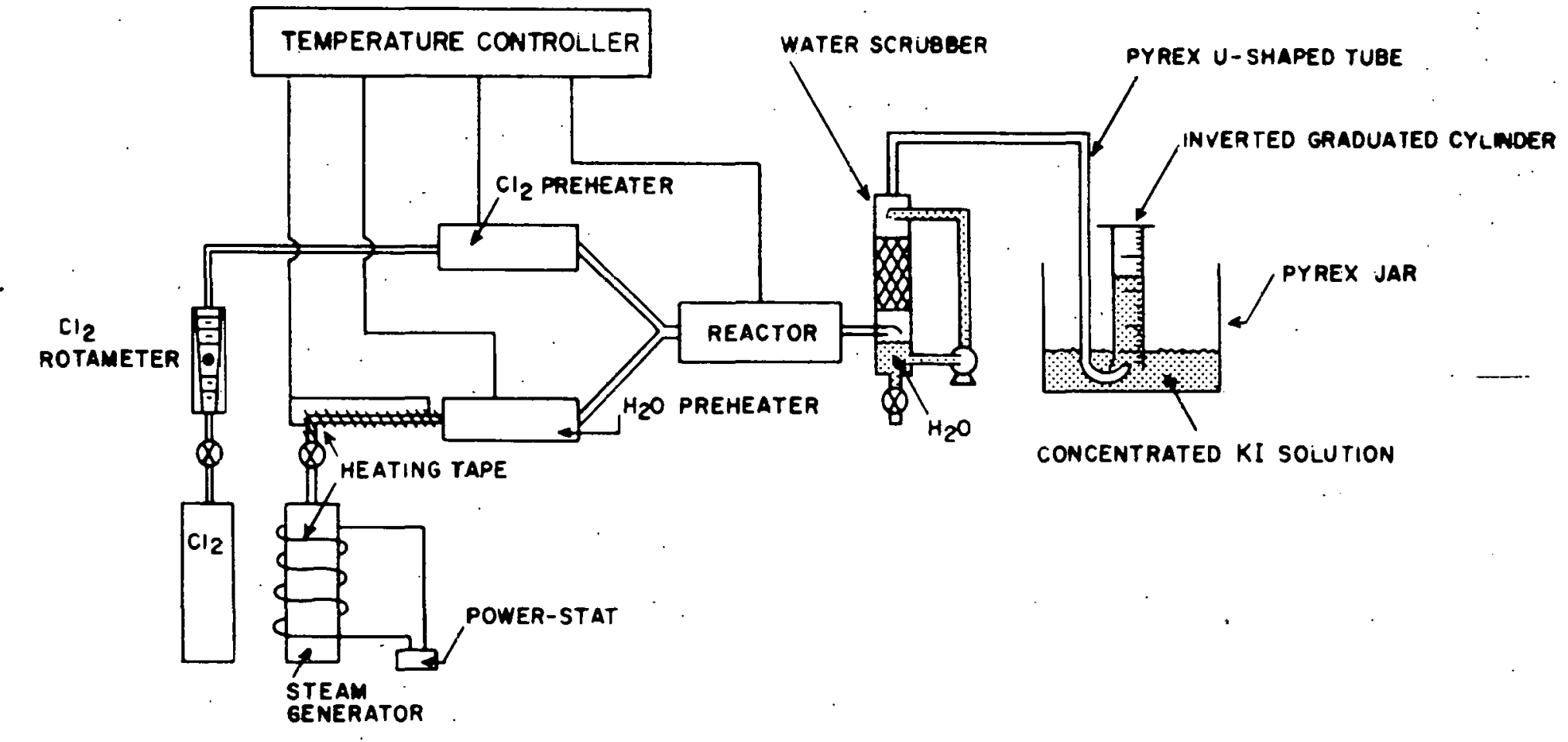

Figure 1. Experimental apparatus 


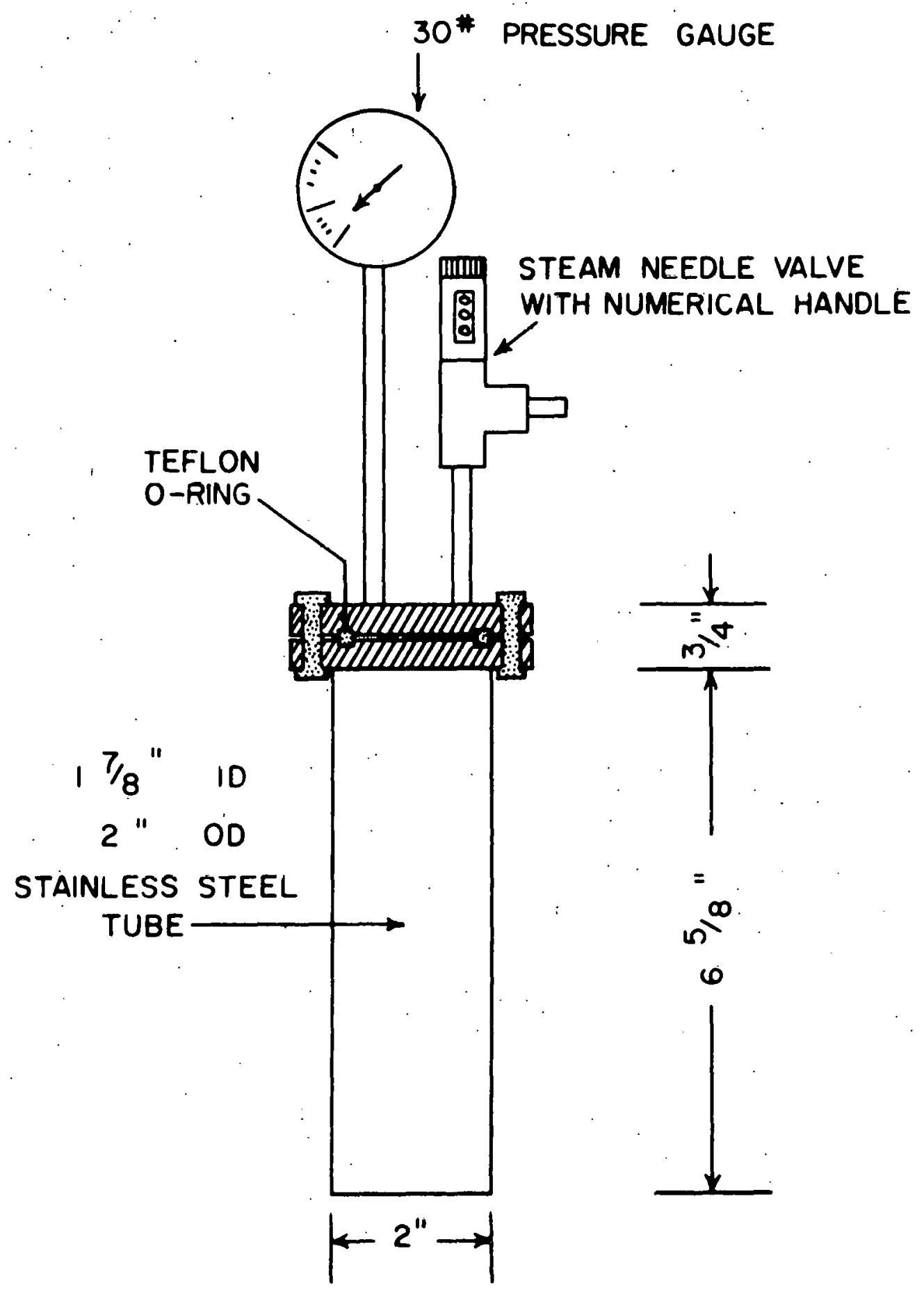

Figure 2. Steam generator i 


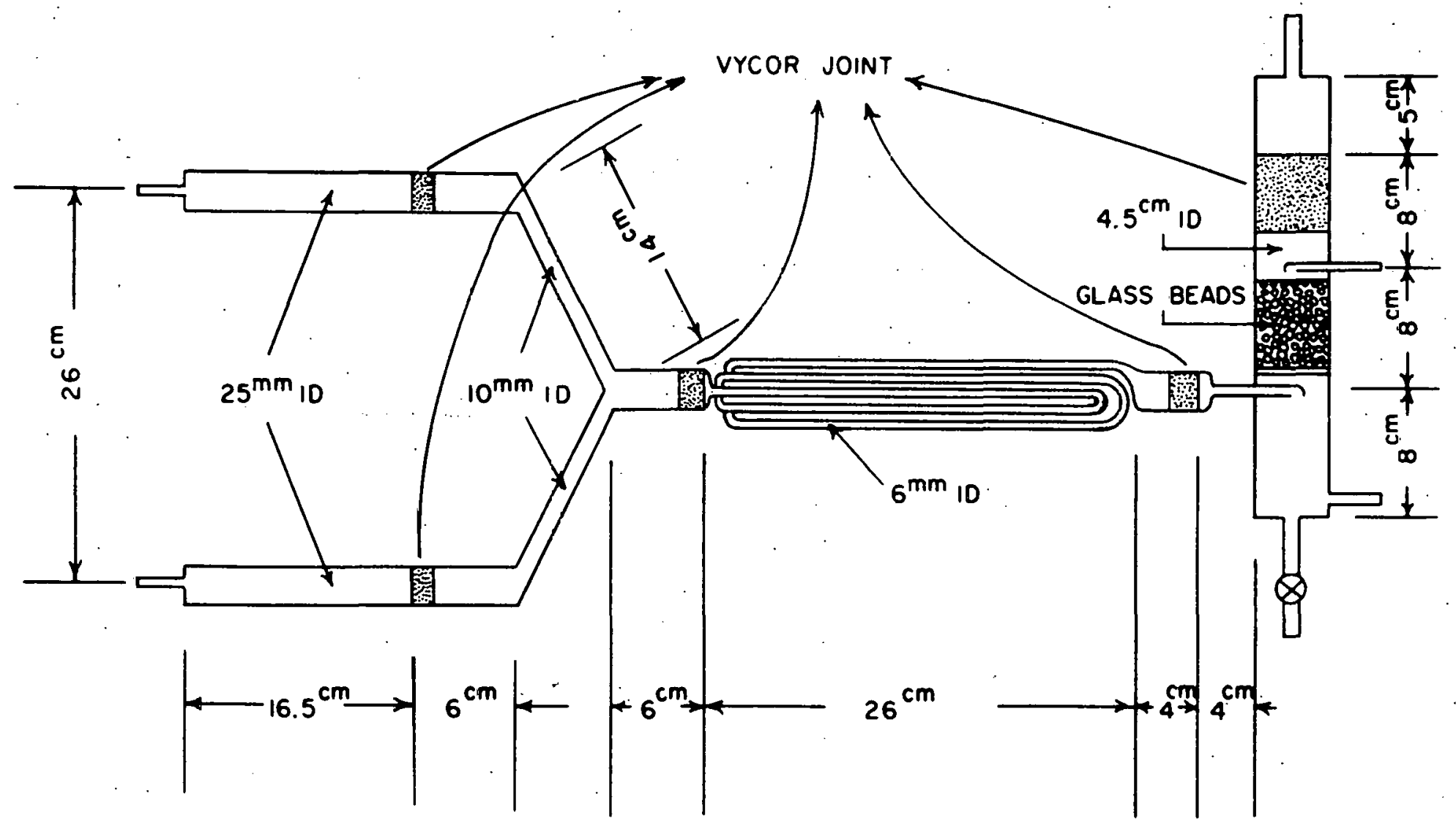

Figure 3. The preheaters, five-pass reactor, and water scrubber 
reactor was put in a 13" long 1-1/4" ID Clam-she11 furnace. The furnace temperatures were on-off controlled by Honeywel1's Brown Protect-0-Vane temperature controller. The Alume1-Chrome1 thermocouples were put in the center of the furnace. A Leeds \& Northrup's millivolt potentiometer was used to calibrate the temperature controller. A Cole-Parmer Instrument Company's variable speed masterflex tubing pump was used to recycle the water in the scrubber. 1/4" Tygon tube was used in the pump line.

The pass from reactor to scrubber was designed so that the product stream could be quenched as fast as possible to prevent a reverse reaction. Glass beads were put in the scrubber to give better contact between gas stream and water. 1/4" Tygon tube connected the out let of the scrubber to a U-shaped pyrex tube. The pyrex tube was immersed in a pyrex jar partly filled with concentrated KI solution.

The line between the preheaters and reactors was wrapped with glass wool insulation material and was directly heated by an infra-red lamp. The temperatures of the preheater-furnaces were always controlled around $150^{\circ} \mathrm{C}$ higher than the reactor-furnace. There existed a temperature drop from the preheaters to the mixing zone. The above arrangements were designed to ineure that the temperature of the gas mixture would be very close to the reactor-furnace temperature when the mixture reached the reactor.

The steam generator and furnaces were heated first. When the pressure in the steam generator reached the required level, the steam valve was opened to let the steam into the system. For each experiment, which usually ran for four hours, the stcam valve was set at a fixed opening. Then the chlorine valve was opened. The steam generator 
was weighed before and after each experiment. The weight difference divided by, the steam valve operating time was the mass flow rate of steam. Ideal gas law was used to convert the mass flow rates into volumetric flow rates. The results were double-checked with the steam table:

It usually took thirty minutes for the whole system to reach steady state. Then a timer and an inverted graduated cylinder filled with $\mathrm{KI}$ solution were used to measure the oxygen evolution rates. Oxygen, hydrochloric acid, unreacted steam and chlorine were present in the product gas stream. The scrubber condensed the steam and absorbed the hydrochloric acid and some chlorine gas. Only oxygen and chlorine entered the U-shaped tube. Then chlorine reacted with potassium iodide $\left(\mathrm{Cl}_{2}+2 \mathrm{I}^{-} \longrightarrow 2 \mathrm{Cl}^{-}+\mathrm{I}_{2}, \mathrm{I}_{2}+\mathrm{I}^{-} \longrightarrow \mathrm{I}_{3}^{-}\right)$(55), so that oxygen was the only gas entering the inverse graduated cylinder. The gas volume in the inverse graduated cylinder was recorded every five minutes. The average oxygen evolution rates were calculated. Usually each run lasted for thirty minutes. The chlorine flow rate was then adjusted to a new value. It took roughly another thirty minutes for the system to regaln steady statc. During each experiment, the steam flow rate was fixed. The space-time was varied only by changing the chlorine flow rate. 


\section{THEORY}

Design Equation for Steady-State P1ug F1ow Reactor (37)

Three assumptions were made for the reactor. First, there was to be no mixing in the axial direction (the direction of flow). Second, there was to be complete mixing in the radial direction. Third, there would be a uniform velocity across the radius. The reactor met the criteria of a plug flow reactor (52). The deviations from the plug flow assumptions will be discussed later (p. 49).

In a plug flow reactor the composition of the fluid varies from point to point along a flow path. Consequently, the material balance for a reaction component must be made for a differential element of volume $\mathrm{dV}$.

$$
\text { Input }=\text { Output }+ \text { Disappearance by Reaction }
$$

Referring to Figure 4:

$$
\begin{aligned}
& \text { Input of } A=F_{A} ; \\
& \text { Output of } A=F_{A}+d F_{A} ; \\
& \text { Disappearance of } \Lambda \text { by reaction (moles/time) } \\
& \quad=\left(-r_{A}\right) d V=\left(\frac{\text { moles } A \text { reacting }}{(t i m e)(\text { volume of fluids) }}\right) \\
& \quad X \text { (volume of differential element); }
\end{aligned}
$$

So Equation (2) becomes

$$
F_{A}=\left(F_{A}+d F_{A}\right)+\left(-r_{A}\right) d V
$$

Because 


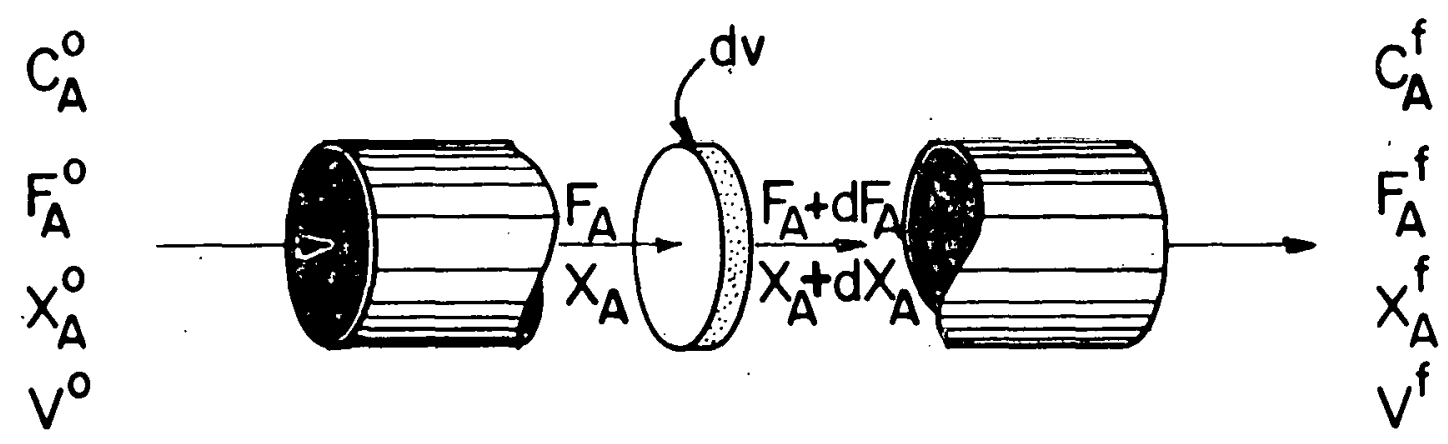

$C_{A}:$ CONCENTRATION OF A (moles/volume)

$F_{A}:$ MOLAL FLOW RATE OF A (moles/time)

$X_{A}:$ FRACTION OF A CONVERTED INTO PRODUCT

$V$ : TOTAL VOLUMETRIC FLOW RATE (volume/time)

SUPERSCRIPT O: CONDITIONS AT THE ENTRANCE SUPERSCRIPT $f$ : CONDITIONS AT THE EXIT

Figure 4. Notation for a plug flow reactor 


$$
F_{A}=F_{A O}\left(1-X_{A}\right)
$$

and

$$
\mathrm{dF}_{\mathrm{A}}=\mathrm{d}\left[\mathrm{F}_{\mathrm{Ao}}\left(1-\mathrm{X}_{\mathrm{A}}\right)\right]=-\mathrm{F}_{\mathrm{Ao}_{\mathrm{o}}} \mathrm{dX_{A }}
$$

Equations (3) and (4) can be combined to give

$$
F_{A o} d_{A}=\left(-r_{A}\right) d V
$$

This is the equation accounting for $A$ in the differential section of reactor of volume $d V$. For the reactor as a whole, Equation (5) must be integrated.

$$
\int_{0}^{V} \frac{d V}{F_{A}^{o}}=\int_{0}^{X_{A}^{f}} \frac{d x_{A}}{\left(-r_{A}\right)}
$$

Since $\mathrm{F}_{\mathrm{A}}^{\mathrm{O}}$ is a constant,

$$
\frac{V}{F_{A}^{o}}=\int_{0}^{X_{A}^{f}} \frac{d X_{A}}{\left(-r_{A}\right)}
$$

and

$$
F_{\mathrm{A}}^{\mathrm{O}}=\mathrm{U}_{\mathrm{O}} \mathrm{C}_{\mathrm{A}}^{\mathrm{O}}
$$

Therefore, $\tau=$ space-time $=$ time required to process one reactor volume

$$
\text { of feed }=\frac{V}{U_{0}}=\frac{F_{A}^{o}}{U_{0}} \int_{0}^{x_{A}^{f}} \frac{{ }^{f} x_{A}}{\left(-r_{A}\right)}=C_{A}^{o} \int_{0}^{x_{A}^{f}} \frac{{ }^{f} x_{A}}{\left(-r_{A}\right)}
$$

\section{Concentration of Each Species Present in the System}

The reverse Deacon reaction may be written in either of the following two forms: 


$$
\begin{array}{ll}
\mathrm{Cl}_{2(\mathrm{~g})}+\mathrm{H}_{2} \mathrm{O}(\mathrm{g}) \frac{\mathrm{k}_{1}}{\underset{\mathrm{k}_{2}}{\rightleftharpoons}} 2 \mathrm{HCL}(\mathrm{g})+\frac{1}{2} \mathrm{O}_{2(\mathrm{~g})} & \text { Reaction I } \\
2 \mathrm{Cl}_{2(\mathrm{~g})}+2 \mathrm{H}_{2} \mathrm{O}(\mathrm{g}) \frac{\mathrm{k}_{1}^{\prime}}{\mathrm{k}_{2}^{\prime}} 4 \mathrm{HCl}_{(\mathrm{g})}+\mathrm{O}_{2(\mathrm{~g})} & \text { Reaction II }
\end{array}
$$

1. If chlorine is the limiting species:

$$
\begin{aligned}
& \mathrm{N}_{1}=\frac{\mathrm{F}_{\mathrm{H}_{2} \mathrm{O}}^{\mathrm{O}}}{\mathrm{F}_{\mathrm{Cl}_{2}}^{\mathrm{O}}}=\frac{\mathrm{C}_{\mathrm{H}_{2} \mathrm{O}}^{\mathrm{O}}}{\mathrm{C}_{\mathrm{Cl}_{2}}^{\mathrm{o}}}
\end{aligned}
$$

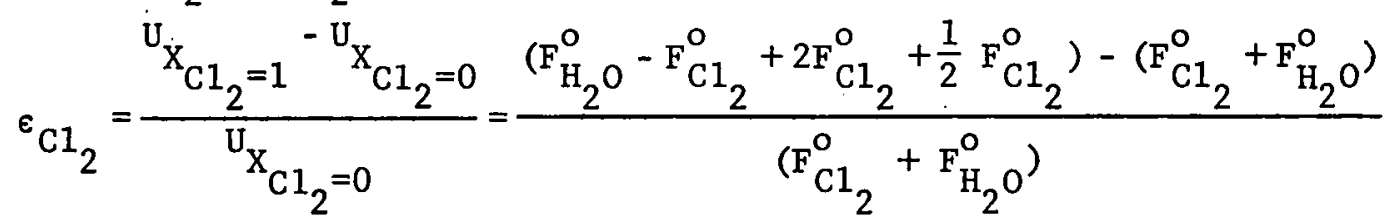

$$
\begin{aligned}
& =\frac{\mathrm{F}_{\mathrm{C} 1_{2}}^{\mathrm{O}}}{2\left(\mathrm{~F}_{\mathrm{Cl} 1_{2}}^{\mathrm{O}}+\mathrm{F}_{\mathrm{H}_{2} \mathrm{O}}^{\mathrm{O}}\right)}=\frac{1}{2\left(1+\mathrm{N}_{1}\right)} \\
& \mathrm{x}_{\mathrm{Cl}_{2}}=1-\frac{\mathrm{C}_{\mathrm{Cl}}}{\mathrm{C}_{\mathrm{Cl}}^{\mathrm{o}}} \\
& \mathrm{U}=\mathrm{U}_{\mathrm{o}}\left[1+\epsilon_{\mathrm{Cl}}{ }_{2} \mathrm{x}_{\mathrm{Cl}}\right]=\mathrm{U}_{\mathrm{o}}\left[1+\frac{\mathrm{X}_{2}}{2\left(1+\mathrm{N}_{1}\right)}\right]=\mathrm{U}_{\mathrm{o}}\left[\frac{2\left(\mathrm{~N}_{1}+1\right)+\mathrm{X}_{\mathrm{Cl}}}{2\left(\mathrm{~N}_{1}+1\right)}\right]
\end{aligned}
$$

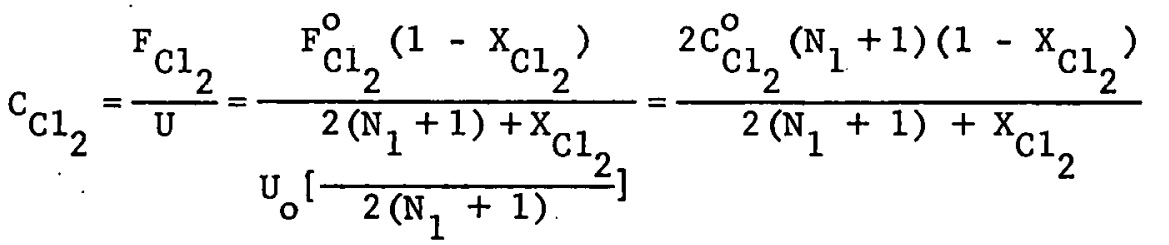

$$
\begin{aligned}
& \mathrm{C}_{\mathrm{H}_{2} \mathrm{O}}=\frac{\mathrm{F}_{\mathrm{H}_{2} \mathrm{O}}}{\mathrm{U}}=\frac{\mathrm{F}_{\mathrm{H}_{2} \mathrm{O}}^{\mathrm{O}}-\mathrm{F}_{\mathrm{Cl}{ }_{2} \mathrm{X}_{\mathrm{C} 1_{2}}}}{2\left(\mathrm{~N}_{1}+1\right)+\mathrm{X}_{\mathrm{C} 1_{2}}}=\frac{2 \mathrm{C}_{\mathrm{C} 1_{2}}^{\mathrm{O}}\left(\mathrm{N}_{1}+1\right)\left(\mathrm{N}_{1}-\mathrm{X}_{\mathrm{C} 1_{2}}\right)}{2\left(\frac{\left.\mathrm{N}_{1}+1\right)+\mathrm{X}_{\mathrm{C} 1_{2}}}{2\left(\mathrm{~N}_{1}+1\right)}\right]}
\end{aligned}
$$




$$
\begin{aligned}
& \mathrm{C}_{\mathrm{HC} 1}=\frac{2 \mathrm{~F}_{\mathrm{Cl}}^{\mathrm{o}} \mathrm{X}_{\mathrm{Cl}}}{2\left(\mathrm{~N}_{1}+1\right)+\mathrm{X}_{\mathrm{Cl}}}=\frac{\left.4 \mathrm{C}_{\mathrm{Cl}_{2}}^{\mathrm{o}}{ }_{\mathrm{N}}+1\right) \mathrm{N}_{\mathrm{Ci}} \mathrm{U}_{2}}{2\left(\mathrm{~N}_{1}+1\right)+\mathrm{X}_{\mathrm{Cl}} \mathrm{N}_{2}} \\
& \mathrm{C}_{\mathrm{O}_{2}}=\frac{\frac{1}{2} \mathrm{~F}_{\mathrm{Cl}_{2}}^{\mathrm{o}} \mathrm{X}_{\mathrm{Cl}}}{2\left(\mathrm{~N}_{1}+1\right)+\mathrm{X}_{\dot{\mathrm{C}} 1_{2}}}=\frac{\mathrm{C}_{\mathrm{Cl}_{2}}^{\mathrm{o}}{ }_{\mathrm{o}}\left[\frac{\left.\mathrm{N}_{1}+1\right) \mathrm{X}_{\mathrm{Cl}}}{2\left(\mathrm{~N}_{1}+1\right)}\right]}{2\left(\mathrm{~N}_{1}+1\right)+\mathrm{X}_{\mathrm{Cl} 1_{2}}} \\
& \mathrm{X}_{\mathrm{H}_{2} \mathrm{O}}^{*}=\frac{\mathrm{C}_{\mathrm{Cl}_{2}}^{\mathrm{o}}{ }^{\mathrm{X}}{ }_{\mathrm{Cl}}}{\mathrm{C}_{\mathrm{H}_{2} \mathrm{O}}^{\mathrm{o}}}=\frac{\mathrm{x}_{\mathrm{Cl}_{2}}}{\mathrm{~N}_{1}}
\end{aligned}
$$

2. If steam is the limiting species:

$$
\begin{aligned}
& \mathrm{N}_{2}=\frac{\mathrm{F}_{\mathrm{Cl} 1_{2}}^{\mathrm{O}}}{\mathrm{F}_{\mathrm{H}_{2} \mathrm{O}}^{\mathrm{O}}}=\frac{\mathrm{C}_{\mathrm{Cl}}^{\mathrm{O}}}{\mathrm{C}_{\mathrm{H}_{2} \mathrm{O}}^{\mathrm{O}}} \\
& \epsilon_{\mathrm{H}_{2} \mathrm{O}}=\frac{\mathrm{U}_{\mathrm{H}_{2} \mathrm{O}=1}^{-\mathrm{J}_{\mathrm{H}_{2} \mathrm{O}=0}}}{\mathrm{U}_{\mathrm{H}_{2} \mathrm{O}=0}}=\frac{\left(\mathrm{F}_{\mathrm{C} 1_{2}}^{\mathrm{O}}-\mathrm{F}_{\mathrm{H}_{2} \mathrm{O}}^{\mathrm{O}}+2 \mathrm{~F}_{\mathrm{H}_{2} \mathrm{O}}^{\mathrm{O}}+\frac{1}{2} \mathrm{~F}_{\mathrm{H}_{2} \mathrm{O}}^{\mathrm{O}}\right)-\left(\mathrm{F}_{\mathrm{H}_{2} \mathrm{O}}^{\mathrm{O}}+\mathrm{F}_{\mathrm{Cl}_{2}}^{\mathrm{O}}\right)}{\left(\mathrm{F}_{\mathrm{H}_{2} \mathrm{O}}^{\mathrm{O}}+\mathrm{F}_{\mathrm{C} \mathrm{I}_{2}}^{\mathrm{O}}\right)} \\
& =\frac{\mathrm{F}_{\mathrm{H}_{2} \mathrm{O}}^{\mathrm{O}}}{2\left(\mathrm{~F}_{\mathrm{H}_{2} \mathrm{O}}^{\mathrm{O}}+\mathrm{F}_{\mathrm{Cl}_{2}}^{\mathrm{O}}\right)}=\frac{1}{2\left(1+\mathrm{N}_{2}\right)} \\
& \mathrm{X}_{\mathrm{H}_{2} \mathrm{O}}=1-\frac{\mathrm{C}_{\mathrm{H}_{2} \mathrm{O}}}{\mathrm{C}_{\mathrm{H}_{2} \mathrm{O}}^{\mathrm{O}}} \\
& \mathrm{U}=\mathrm{U}_{\mathrm{o}}\left[1+\epsilon_{\mathrm{H}_{2} \mathrm{O}} \mathrm{X}_{\mathrm{H}_{2} \mathrm{O}}\right]=\mathrm{U}_{\mathrm{o}}\left[1+\frac{\mathrm{X}_{\mathrm{H}_{2} \mathrm{O}}}{2\left(1+\mathrm{N}_{2}\right)}\right]=\mathrm{U}_{\mathrm{o}}\left[\frac{2\left(\mathrm{~N}_{2}+1\right)+\mathrm{X}_{\mathrm{H}_{2} \mathrm{O}}}{2\left(\mathrm{~N}_{2}+1\right)}\right]
\end{aligned}
$$




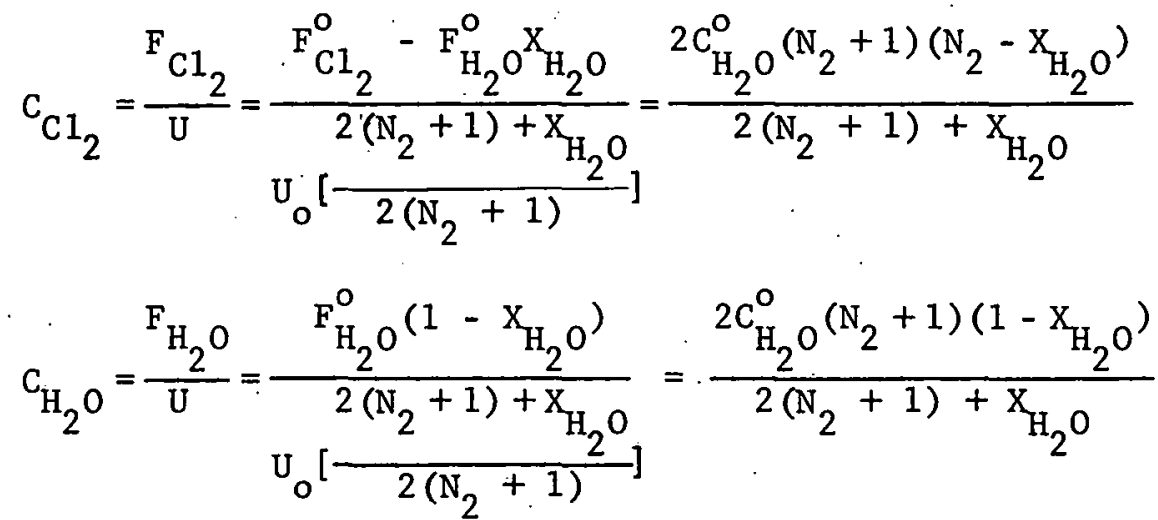

$$
\begin{aligned}
& \mathrm{C}_{\mathrm{HC} 1}=\frac{2 \mathrm{~F}_{\mathrm{H}_{2} \mathrm{O}_{\mathrm{H}_{2} \mathrm{O}}^{\mathrm{O}}}}{2\left(\mathrm{~N}_{2}+1\right)+\mathrm{X}_{\mathrm{H}_{2} \mathrm{O}}}=\frac{4 \mathrm{C}_{\mathrm{H}_{2} \mathrm{O}}^{\mathrm{O}}\left(\mathrm{N}_{2}+1\right) \mathrm{X}_{\mathrm{H}_{2} \mathrm{O}}}{2\left(\mathrm{~N}_{2}+1\right)+\mathrm{X}_{\mathrm{H}_{2} \mathrm{O}}} \\
& \mathrm{C}_{2}=\frac{\left.\frac{1}{2} \mathrm{~F}_{\mathrm{H}_{2} \mathrm{O}_{\mathrm{H}_{2} \mathrm{O}}^{\mathrm{O}}}=\frac{\mathrm{C}_{\mathrm{H}_{2} \mathrm{O}}^{\mathrm{O}}\left(\mathrm{N}_{2}+1\right) \mathrm{X}_{\mathrm{H}_{2} \mathrm{O}}}{2\left(\mathrm{~N}_{2}+1\right)+\mathrm{X}_{\mathrm{H}_{2} \mathrm{O}}}\right]}{2\left(\frac{\left.\mathrm{N}_{2}+1\right)+\mathrm{X}_{\mathrm{H}_{2} \mathrm{O}}}{2\left(\mathrm{~N}_{2}+1\right)}\right]} \\
& \mathrm{X}_{\mathrm{C} 1_{2}}^{*}=\frac{\mathrm{C}_{\mathrm{H}_{2} \mathrm{O}}^{\mathrm{O}} \cdot \mathrm{H}_{2} \mathrm{O}}{\mathrm{C}_{\mathrm{Cl}}^{\mathrm{o}}} \cdot=\frac{\mathrm{x}_{\mathrm{H}_{2} \mathrm{O}}}{\mathrm{N}_{2}}
\end{aligned}
$$

Rate and Design Equations Neglecting Reverse Reaction

If the reverse reactions of Reactions I and II can be neglected, the reaction rate equations are:

$$
\begin{aligned}
& \text { Rate }=\left(-\mathrm{r}_{\mathrm{Cl}_{2}}\right)=\left(-\mathrm{r}_{\mathrm{H}_{2} \mathrm{O}}\right)=\mathrm{k}_{1} \mathrm{C}_{\mathrm{Cl}}^{\mathrm{a}} \cdot \mathrm{C}_{\mathrm{H}_{2} \mathrm{O}}^{\mathrm{b}} \text { for Reaction I } \\
& \text { Rate }=\left(-\mathrm{r}_{\mathrm{Cl}_{2}}\right)=\left(-\mathrm{r}_{\mathrm{H}_{2} \mathrm{O}}\right)=\mathrm{k}_{1}^{\prime} \mathrm{C}_{\mathrm{Cl}}^{\mathrm{a}} \cdot \mathrm{C}_{\mathrm{H}_{2} \mathrm{O}}^{\mathrm{b}} \text { for Reaction II }
\end{aligned}
$$


1. If chlorine is the limiting species:

The derivation for Reaction II is the same as the following derivation, except $k_{1}^{\prime}$ should be used instead of $k_{1}$.

Equation (6) becomes

$$
\tau=c_{C 1}^{o} \int_{0}^{\mathrm{X}_{\mathrm{C} 1}^{\mathrm{f}}} \frac{{ }^{\mathrm{dX} \mathrm{X}_{2}}}{\left(-\mathrm{r}_{\mathrm{C} 1_{2}}\right)}
$$

Inserting Equations (11) and (12) into Equation (25) gives

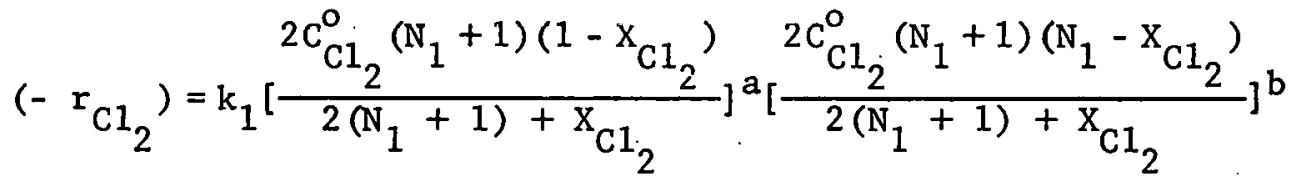

$$
\begin{aligned}
& =\mathrm{k}_{1}\left[\mathrm{C}_{\mathrm{Cl} 1_{2}}^{\mathrm{o}}\right]^{\mathrm{a}+\mathrm{b}}\left[\frac{2\left(\mathrm{~N}_{1}+1\right)}{2\left(\mathrm{~N}_{1}+1\right)+\mathrm{X}_{\mathrm{A}}}\right]^{\mathrm{a}+\mathrm{b}}\left(1-\mathrm{X}_{\mathrm{Cl} 1_{2}}\right)^{\mathrm{a}} \cdot\left(\mathrm{N}_{1}-\mathrm{X}_{\mathrm{Cl} 1_{2}}\right)^{\mathrm{b}}
\end{aligned}
$$

Combining Equations (27), and (28) gives

$$
\tau=\mathrm{C}_{\mathrm{C} 1_{2}}^{\mathrm{o}} \int_{0}^{\mathrm{x}} \mathrm{X}_{2}^{\mathrm{f}}
$$

$$
\frac{\mathrm{dX}_{\mathrm{Cl}}}{\mathrm{k}_{1}\left[\mathrm{C}_{\mathrm{Cl} 1_{2}}^{\mathrm{o}}\right]^{\mathrm{a}+\mathrm{b}} \cdot\left[\frac{2\left(\mathrm{~N}_{1}+1\right)}{\left.2\left(\mathrm{~N}_{1}+1\right)+\mathrm{X}_{\mathrm{Cl}}\right]^{\mathrm{a}+\mathrm{b}} \cdot\left(1-\mathrm{X}_{\mathrm{C1} 1_{2}}\right)^{\mathrm{a}} \cdot\left(\mathrm{N}_{1}-\mathrm{X}_{\mathrm{Cl} 1_{2}}\right)^{\mathrm{b}}}\right.}
$$

2. If steam is the limiting species:

The derivation for Reactiun II is the same as the tollowing derivation, except $k_{1}^{\prime}$ should be used instead of $k_{1}$.

Equation (6) becomes 


$$
\tau=\mathrm{C}_{\mathrm{H}_{2} \mathrm{O}}^{\mathrm{O}} \int_{0}^{\mathrm{X}_{\mathrm{H}}^{\mathrm{f}} \mathrm{O}} \cdot \frac{\mathrm{dX}_{\mathrm{H}_{2} \mathrm{O}}}{\left(-\mathrm{r}_{\mathrm{H}_{2} \mathrm{O}}\right)}
$$

Inserting Equations (20) and (21) into Equation (25) gives

$$
\begin{aligned}
& \left(-\mathrm{r}_{\left.\mathrm{H}_{2} \mathrm{O}\right)}=\mathrm{k}_{1} \cdot\left[\frac{2 \mathrm{C}_{\mathrm{H}_{2} \mathrm{O}}^{\mathrm{O}}\left(\mathrm{N}_{2}+1\right)\left(\mathrm{N}_{2}-\dot{\mathrm{X}}_{\mathrm{H}_{2} \mathrm{O}}\right)}{2\left(\mathrm{~N}_{2}+1\right)+\mathrm{X}_{\mathrm{H}_{2} \mathrm{O}}}\right]^{\mathrm{a}}\right. \\
& \quad\left[\frac{2 \mathrm{C}_{\mathrm{H}_{2} \mathrm{O}}^{\mathrm{O}}\left(\mathrm{N}_{2}+1\right)\left(1-\mathrm{X}_{\mathrm{H}_{2} \mathrm{O}}\right)}{2\left(\mathrm{~N}_{2}+1\right)+\mathrm{X}_{\mathrm{H}_{2} \mathrm{O}} \mathrm{b}}\right]^{\mathrm{O}} \\
& =\mathrm{k}_{1} \cdot\left[\mathrm{C}_{\mathrm{H}_{2} \mathrm{O}}^{\mathrm{O}}\right]^{\mathrm{a}+\mathrm{b}} \cdot\left[\frac{2\left(\mathrm{~N}_{2}+1\right)}{2\left(\mathrm{~N}_{2}+1\right)+\mathrm{X}_{\mathrm{H}_{2} \mathrm{O}}}\right]^{\mathrm{a}+\mathrm{b}} \cdot\left(\mathrm{N}_{2}-\mathrm{X}_{\mathrm{H}_{2} \mathrm{O}}\right)^{\mathrm{a}} \cdot\left(1-\mathrm{X}_{\mathrm{H}_{2} \mathrm{O}}\right)^{\mathrm{b}}
\end{aligned}
$$

Combining Equations (30) and (31) gives

$$
\tau=\mathrm{C}_{\mathrm{H}_{2} \mathrm{O}}^{\mathrm{o}} \int_{0}^{\mathrm{X}} \int_{\mathrm{H}_{2} \mathrm{O}}^{\mathrm{f}}
$$

$$
\frac{\mathrm{dX}_{\mathrm{H}_{2} \mathrm{O}}}{\mathrm{k}_{1}\left[\mathrm{C}_{\mathrm{H}_{2} \mathrm{O}}^{\mathrm{O}}\right]^{\mathrm{a}+\mathrm{b}} \cdot\left[\frac{2\left(\mathrm{~N}_{2}+1\right)}{2\left(\mathrm{~N}_{2}+1\right)+\mathrm{X}_{\mathrm{H}_{2} \mathrm{O}}}\right]^{\mathrm{a}+\mathrm{b}} \cdot\left(\mathrm{N}_{2}-\mathrm{X}_{\mathrm{H}_{2} \mathrm{O}}\right)^{\mathrm{a}} \cdot\left(1-\mathrm{X}_{\mathrm{H}_{2} \mathrm{O}}\right)^{\mathrm{b}}}
$$

3. Comparison of Equations (29) and (32):

The following derivation will prove that Equation (32) is actually a different form of Equation (29).

Combining Equations (7), (15), and (29) gives 


$$
\begin{aligned}
& \tau=\frac{\mathrm{C}_{2}^{\mathrm{O}} \mathrm{O}}{\mathrm{N}_{1}} \int_{0}^{\left(\mathrm{X}_{\mathrm{H}_{2} \mathrm{O}}^{*}\right)^{\mathrm{f}}} \\
& \mathrm{N}_{1} \cdot \mathrm{dx}_{\mathrm{H}_{2} \mathrm{O}}^{*} \\
& \mathrm{k}_{1} \cdot\left(\frac{\mathrm{C}_{2}^{\mathrm{O}}}{\mathrm{N}_{1}}\right)^{\mathrm{a}+\mathrm{b}} \cdot\left[\frac{2\left(\mathrm{~N}_{1}+1\right)}{2\left(\mathrm{~N}_{1}+1\right)+\mathrm{N}_{1} \mathrm{x}_{\mathrm{H}_{2} \mathrm{O}}^{*}}\right]^{\mathrm{a}+\mathrm{b}} \cdot\left(1-\mathrm{N}_{1} \mathrm{x}_{\mathrm{H}_{2} \mathrm{O}}^{*}\right)^{\mathrm{a}} \cdot\left(\mathrm{N}_{1}-\mathrm{N}_{1} \mathrm{X}_{\mathrm{H}_{2} \mathrm{O}}^{*}\right)^{\mathrm{b}} \\
& =\frac{\mathrm{C}_{\mathrm{H}_{2} \mathrm{O}}^{\mathrm{o}}}{\mathrm{N}_{1}} \int_{0}^{\left(\mathrm{x}_{\mathrm{H}_{2} \mathrm{O}}^{*}\right)^{\mathrm{f}}} \\
& \frac{\mathrm{N}_{1}^{\mathrm{a}} \cdot \mathrm{N}_{1} \cdot \mathrm{dx}_{\mathrm{H}_{2} \mathrm{O}}^{*}}{\mathrm{k}_{1} \cdot\left(\mathrm{C}_{\mathrm{H}_{2} \mathrm{O}}^{\mathrm{o}}\right)^{\mathrm{a+b}} \cdot\left[\frac{2\left(\mathrm{~N}_{1}+1\right)}{2\left(\mathrm{~N}_{1}+1\right)+\mathrm{N}_{1} \mathrm{X}_{\mathrm{H}_{2} \mathrm{O}}^{*}}\right]^{\mathrm{a+b}} \cdot\left(1-\mathrm{N}_{1} \mathrm{x}_{\mathrm{H}_{2} \mathrm{O}}^{*}\right)^{\mathrm{a}} \cdot\left(1-\mathrm{X}_{\mathrm{H}_{2} \mathrm{O}}^{*}\right)^{\mathrm{b}}}
\end{aligned}
$$

Combining Equations (71) and (16) gives

$$
\mathrm{N}_{1}=\frac{1}{\mathrm{~N}_{2}}
$$

Inserting Equation (34) into Equation (33) gives

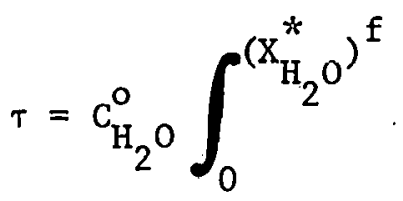

$$
\begin{aligned}
& \frac{\mathrm{dx}_{\mathrm{H}_{2} \mathrm{O}}^{*}}{\mathrm{k}_{1} \cdot\left(\mathrm{C}_{\mathrm{H}_{2} \mathrm{O}}^{\mathrm{O}}\right)^{\mathrm{a+b}} \cdot\left[\frac{2\left(\mathrm{~N}_{2}+1\right)}{2\left(\mathrm{~N}_{2}+1\right)+\mathrm{X}_{\mathrm{H}_{2} \mathrm{O}}^{*}}\right]^{\mathrm{a}+\mathrm{b}} \cdot\left(\mathrm{N}_{2}-\mathrm{x}_{\mathrm{H}_{2} \mathrm{O}}^{*}\right)^{\mathrm{a}} \cdot\left(1-\mathrm{X}_{\mathrm{H}_{2} \mathrm{O}}^{*}\right)^{\mathrm{b}}}
\end{aligned}
$$

From Equation (15), 


$$
\mathrm{x}_{\mathrm{H}_{2} \mathrm{O}}^{*}=\frac{\mathrm{C}_{\mathrm{C} 1_{2}}^{\mathrm{o}}}{\mathrm{C}_{\mathrm{H}_{2} \mathrm{O}}^{\mathrm{o}}} \cdot \mathrm{x}_{\mathrm{Cl}_{2}}=\frac{\mathrm{C}_{\mathrm{Cl}}^{\mathrm{o}}}{\mathrm{c}_{\mathrm{H}_{2} \mathrm{O}}^{\circ}} \cdot \frac{2 \mathrm{U}_{2}}{\mathrm{U}_{\mathrm{Cl}_{2}}^{\mathrm{o}}}
$$

And $\quad \mathrm{X}_{\mathrm{H}_{2} \mathrm{O}}=\frac{2 \mathrm{U}_{2}}{\mathrm{U}_{\mathrm{H}_{2} \mathrm{O}}^{\mathrm{O}}}$

Applying the ideal gas law,

$$
\begin{aligned}
& \mathrm{C}_{\mathrm{Cl} 2}^{\circ}=\frac{\mathrm{P}_{\mathrm{Cl}}^{\mathrm{o}}}{\mathrm{RT}}=\frac{\mathrm{P}_{\mathrm{t}} \cdot\left(\frac{\mathrm{U}_{\mathrm{C} 1_{2}}^{\circ}}{\mathrm{U}_{\mathrm{H}_{2} \mathrm{O}}^{\mathrm{o}}+\mathrm{U}_{\mathrm{C} 1_{2}}^{\mathrm{o}}}\right)}{\mathrm{RT}} \\
& \mathrm{C}_{\mathrm{H}_{2} \mathrm{O}}^{\mathrm{O}}=\frac{\mathrm{P}_{\mathrm{H}_{2} \mathrm{O}}^{\mathrm{O}}}{\mathrm{RT}}=\frac{\mathrm{P}_{\mathrm{t}} \cdot\left(\frac{\mathrm{U}_{\mathrm{H}_{2} \mathrm{O}}^{\mathrm{O}}}{\mathrm{U}_{\mathrm{H}_{2} \mathrm{O}}^{\mathrm{O}}+\mathrm{U}_{\mathrm{C}{ }_{2}}^{\mathrm{O}}}\right)}{\mathrm{RT}}
\end{aligned}
$$

Dividing Equation (38) by Equation (39) gives

$$
\frac{\mathrm{C}_{\mathrm{C} 1_{2}}^{\mathrm{o}}}{\mathrm{C}_{\mathrm{H}_{2} \mathrm{O}}^{\mathrm{O}}}=\frac{\mathrm{U}_{\mathrm{C} 1_{2}}^{\mathrm{o}}}{\mathrm{U}_{\mathrm{H}_{2} \mathrm{O}}^{\mathrm{o}}}
$$

Combining Equations (36), (37), and (40) gives

$$
\mathrm{X}_{\mathrm{H}_{2} \mathrm{O}}=\mathrm{x}_{\mathrm{H}_{2} \mathrm{O}}^{*}
$$

Then Equation (35) becomes exactly the same as Equation (32). Therefore, when Equation (32) is applied to the steam-1imiting cases the results are the same as applying Equation (35). 
Two Special Cases of the Design Equations Neglecting Reverse Reaction.

1. $a=1, b=0$

This could happen to both Reactions I and II. The derivation for Reaction II is the same as the following derivation except $k_{1}^{\prime}$ should be used instead of $k_{1}$.

Equation (29) becomes

$$
\begin{aligned}
& \tau=\mathrm{C}_{\mathrm{Cl}}^{\mathrm{o}} \int_{0}^{\mathrm{x}_{\mathrm{Cl} 1_{2}}^{\mathrm{f}}} \frac{\mathrm{dx}_{\mathrm{Cl}}}{\mathrm{k}_{1} \cdot \mathrm{C}_{\mathrm{Cl} 1_{2}}^{\mathrm{o}}\left[\frac{2\left(\mathrm{~N}_{1}+1\right)}{2\left(\mathrm{~N}_{1}+1\right)+\mathrm{x}_{\mathrm{Cl}}}\right] \cdot\left(1-\mathrm{x}_{\mathrm{Cl}}\right)} \\
& =\frac{1}{2 \mathrm{k}_{1}\left(\mathrm{~N}_{1}+1\right)} \int_{0}^{\mathrm{X}_{\mathrm{Cl}}^{\mathrm{f}}}\left[-1+\frac{2 \mathrm{~N}_{1}+3}{1-\mathrm{X}_{\mathrm{Cl}}{ }_{2}}\right] \mathrm{dx} \mathrm{Cl}_{2} \\
& =\frac{-\mathrm{x}_{\mathrm{Cl}}^{\mathrm{f}}-\left(2 \mathrm{~N}_{1}+3\right) \ln \left(1-\mathrm{x}_{\mathrm{Cl}}^{\mathrm{f}}\right)}{2 \mathrm{k}_{1}\left(\mathrm{~N}_{1}+1\right)} \text {. } \\
& \text { Let } \quad \mathrm{x}=\frac{-\mathrm{x}_{\mathrm{Cl} 1_{2}}^{\mathrm{f}}-\left(2 \mathrm{~N}_{1}+3\right) \ln \left(1-\mathrm{x}_{\mathrm{C} 1_{2}}^{\mathrm{f}}\right)}{2\left(\mathrm{~N}_{1}+1\right)} \\
& \text { Then } \quad x=k_{1} \tau \\
& \text { 2. } a=2, b=1
\end{aligned}
$$

This could only happen to Reaction II.

Equation (29) becomes

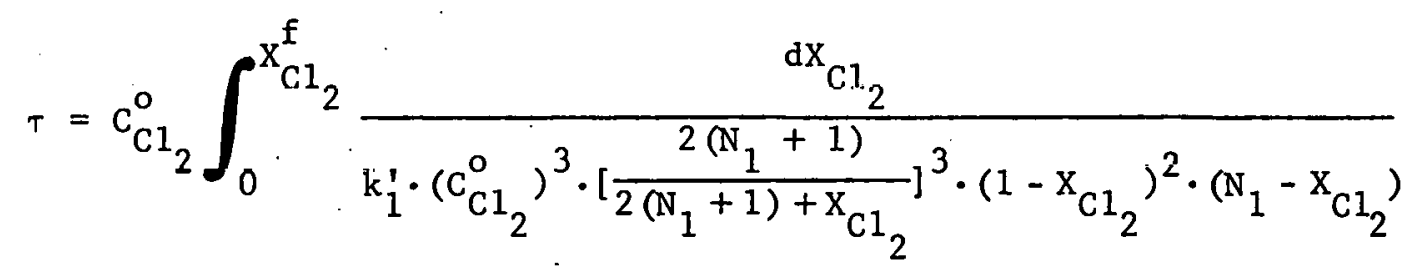




$$
\begin{aligned}
& =\frac{1}{k_{1}^{\prime} \cdot\left[\mathrm{C}_{\mathrm{Cl} 1_{2}}^{\mathrm{j}}\right]^{2} \cdot\left[2\left(\mathrm{~N}_{1}+1\right)\right]^{3}} \int_{0}^{\mathrm{C}_{2} \mathrm{f}} \\
& {\left[-1+\frac{\left(7 \mathrm{~N}_{1}+8\right) \mathrm{x}_{\mathrm{Cl}}^{2}+\left(12 \mathrm{~N}_{1}^{2}+22 \mathrm{~N}_{1}+11\right) \mathrm{x}_{\mathrm{Cl}}+\left(8 \mathrm{~N}_{1}^{3}+24 \mathrm{~N}_{1}^{2}+25 \mathrm{~N}_{1}+8\right)}{\left(1-\mathrm{X}_{\mathrm{Cl}}\right)^{2}\left(\mathrm{~N}_{1}-\mathrm{x}_{\mathrm{Cl}}\right)}\right]}
\end{aligned}
$$

$\mathrm{dX}_{\mathrm{Cl}}$

$$
\begin{aligned}
& =\frac{1}{k_{1}^{\prime} \cdot\left[C_{C 1}^{o}\right]^{2}\left[2\left(N_{1}+1\right)\right]^{3}}\left\{-x_{C 1_{2}}^{f}+\frac{20 N_{1}^{3}+60 N_{1}^{2}+45 N_{1}}{\left(N_{1}-1\right)^{2}} \ln \left(1-x_{C 1_{2}}^{f}\right)\right. \\
& +\frac{8 N_{1}^{3}+36 N_{1}^{2}+54 N_{1}+27}{N_{1}=1}\left(\frac{x_{C 1}^{f}}{1-x_{C 1}^{f}}\right) \\
& \left.-\frac{27 N_{1}^{3}+54 N_{1}^{2}+36 N_{1}+8}{\left(N_{1}-1\right)^{2}} \ln \left(\frac{N_{1}-x_{C 1}^{f}}{N_{1}}\right)\right\}
\end{aligned}
$$

Let

$$
\begin{gathered}
\mathrm{X}=\frac{1}{\left[\mathrm{C}_{\mathrm{Cl} 1_{2}}^{\mathrm{o}}\right]^{2} \cdot\left[2\left(\mathrm{~N}_{1}+1\right)\right]^{3}}\left\{-\mathrm{x}_{\mathrm{C} 1_{2}}^{\mathrm{f}}+\frac{5 \mathrm{~N}_{1}\left(2 \mathrm{~N}_{1}+3\right)^{2}}{\left(\mathrm{~N}_{1}-1\right)^{2}} \ln \left(1-\mathrm{x}_{\mathrm{Cl} 1_{2}}^{\mathrm{f}}\right)\right. \\
\left.+\frac{\left(2 \mathrm{~N}_{1}+3\right)^{3} \cdot \mathrm{x}_{\mathrm{C} 1_{2}}^{\mathrm{f}}}{\left(\mathrm{N}_{1}-1\right)\left(1-\mathrm{x}_{\mathrm{Cl}}\right)}-\frac{\left(3 \mathrm{~N}_{1}+2\right)^{3}}{\left(\mathrm{~N}_{1}-1\right)^{2}} \ln \left(\frac{\mathrm{N}_{1}-\mathrm{x}_{\mathrm{Cl}} \mathrm{N}_{1}}{\mathrm{~N}_{1}}\right)\right\}
\end{gathered}
$$

Then

$$
x=k_{1}^{\prime} \tau
$$

A plot of $\mathrm{X}$ versus $\tau$ will give a straight line of slope $k_{1}$ or slupe $k_{1}$, as shown in Equations (44) and (47), if the correct rate expression has been chosen. 
Rate and Design Equations Including Reverse Reaction

When the reaction reaches equilibrium, the rate of the forward reaction should equal the rate of the reverse reaction. If the forward reaction rate equation is set, the reverse reaction rate equation can be derived by using the equilibrium constant expression. Then the total reaction rate equation is the comblnation of the forward and reverse reaction rate equations.

For Reaction I,

$$
\mathrm{K}_{\text {eq. }}=\text { equilibrium constant }=\frac{\mathrm{k}_{1}}{\mathrm{k}_{2}}=\frac{\left[\mathrm{C}_{\mathrm{HCl} \text {, eq. }}\right]^{2}\left[\mathrm{C}_{\mathrm{O}_{2}, \text { eq. }}\right]^{1 / 2}}{\left[\mathrm{C}_{\mathrm{Cl}} \text {, eq. } \cdot\left[\mathrm{C}_{\mathrm{H}_{2} \mathrm{O} \text {, eq. }}\right]\right.}
$$

For Reaction II,

$$
\mathrm{K}_{\text {eq. }}^{\prime}=\text { equilibrium constant }=\frac{\mathrm{k}_{1}^{\prime}}{\mathrm{k}_{2}^{\prime}}=\frac{\left[\mathrm{C}_{\mathrm{HCl}, \text { eq. }}\right]^{4}\left[\mathrm{C}_{\mathrm{O}_{2}, \text { eq. }}\right]}{\left[\mathrm{C}_{\mathrm{C} 1_{2}, \text { eq. }}\right]^{2}\left[\mathrm{C}_{\mathrm{H}_{2} \mathrm{O}, \text { eq. }}\right]^{2}}
$$

1. If the forward reaction rate equation is

$$
\text { Rate }=\mathrm{k}_{1} \mathrm{C}_{\mathrm{Cl}_{2}} \text { or Rate }=\mathrm{k}_{1}^{\prime} \mathrm{C}_{\mathrm{Cl}_{2}} \text { : }
$$

a. For Reaction. I

$$
\text { Rate }=\left(-\mathrm{r}_{\mathrm{Cl}_{2}}\right)=\mathrm{k}_{1} \cdot \mathrm{C}_{\mathrm{Cl}_{2}}-\mathrm{k}_{2} \frac{\mathrm{C}_{\mathrm{HCl} \mathrm{O}_{2}}^{2}}{\mathrm{C}_{\mathrm{H}_{2} \mathrm{O}}^{1 / 2}}
$$

Conbinting Equations (11), (12), (13), (14) and (bU) gives 


$$
\begin{aligned}
& \left(-\mathrm{r}_{\mathrm{Cl}_{2}}\right)=\mathrm{k}_{1} \cdot \frac{2 \mathrm{C}_{\mathrm{Cl}}^{\mathrm{O}}\left(\mathrm{N}_{1}+1\right)\left(1-\mathrm{x}_{\mathrm{Cl}}\right)}{2\left(\mathrm{~N}_{1}+1\right)+\mathrm{x}_{\mathrm{Cl}}{ }_{2}}
\end{aligned}
$$

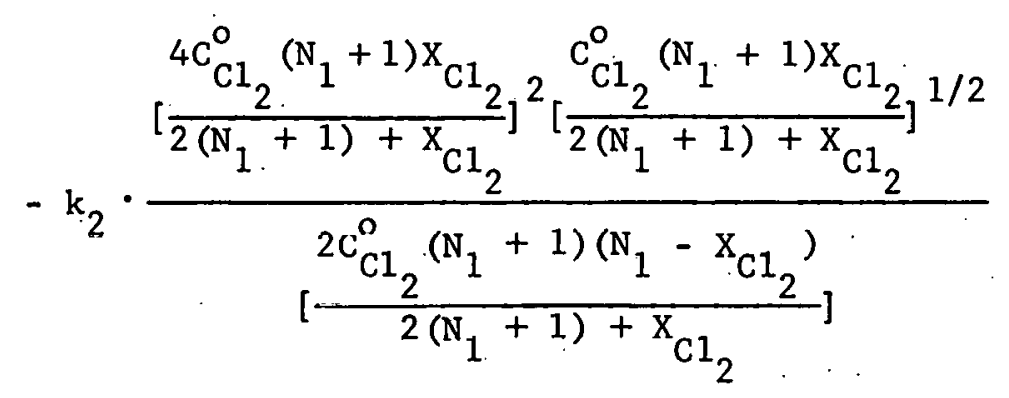

After rearranging and using Equation (48),

$$
\begin{aligned}
& \left(-\mathrm{r}_{\mathrm{Cl}}\right)=\frac{2 \mathrm{k}_{1} \mathrm{C}_{\mathrm{Cl}}^{\mathrm{o}}\left(\mathrm{N}_{1}+1\right)}{2\left(\mathrm{~N}_{1}+1\right)+\mathrm{x}_{\mathrm{Cl}}} \\
& \left\{1-\mathrm{x}_{\mathrm{C} 1_{2}}-\frac{4\left[\mathrm{C}_{\mathrm{Cl}}^{\mathrm{o}}\right]^{1 / 2} \cdot\left(\mathrm{N}_{1}+1\right)^{1 / 2} \cdot \mathrm{x}_{\mathrm{Cl}}^{5 / 2}}{\mathrm{~K}_{\text {eq. }}\left[2\left(\mathrm{~N}_{1}+1\right)+\mathrm{x}_{\mathrm{Cl}}\right]^{1 / 2} \cdot\left(\mathrm{N}_{1}-\mathrm{x}_{\mathrm{C} 1_{2}}\right)}\right\}
\end{aligned}
$$

Combining Equations (52) and (27), and rearranging

$$
\begin{aligned}
& \tau=\frac{1}{2 k_{1}\left(N_{1}+1\right)} \int_{0}^{\mathrm{X}_{\mathrm{Cl}} \mathrm{f}}\left[2\left(\mathrm{~N}_{1}+1\right)+\mathrm{x}_{\mathrm{Cl}{ }_{2}}\right] \\
& \left\{1-\mathrm{x}_{\mathrm{Cl}{ }_{2}}-\frac{4\left[\mathrm{C}_{\mathrm{Cl}}^{\mathrm{o}}\right]^{1 / 2} \cdot\left(\mathrm{N}_{1}+1\right)^{1 / 2} \cdot \mathrm{x}_{\mathrm{Cl}}^{5 / 2}}{\mathrm{~K}_{\mathrm{eq}} \cdot\left[2\left(\mathrm{~N}_{1}+1\right)+\mathrm{x}_{\mathrm{Cl}}\right]^{1 / 2} \cdot\left(\mathrm{N}_{1}-\mathrm{x}_{\mathrm{Cl} 1_{2}}\right)}\right\}^{-1} \mathrm{dx} \mathrm{Cl}_{2}
\end{aligned}
$$

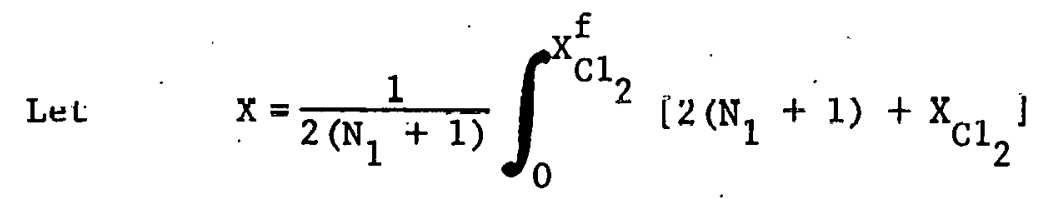




$$
\left\{1-\mathrm{x}_{\mathrm{Cl}}-\frac{4\left[\mathrm{C}_{2}{ }_{2}\right]^{1 / 2} \cdot\left(\mathrm{N}_{1}+1\right)^{1 / 2} \cdot \mathrm{x}_{\mathrm{Cl}}^{5 / 2}}{\mathrm{~K}_{\text {eq. }} \cdot\left[2\left(\mathrm{~N}_{1}+1\right)+\mathrm{x}_{\mathrm{Cl}_{2}}\right]^{1 / 2} \cdot\left(\mathrm{N}_{1}-\mathrm{x}_{\mathrm{Cl}}\right)}\right\}^{-1} \mathrm{dx}_{\mathrm{Cl}_{2}}
$$

Then

$$
\dot{x}=k_{1} \tau
$$

b. For Reaction II

$$
\text { Rate }=\left(-\mathrm{r}_{\mathrm{Cl}_{2}}\right)=\mathrm{k}_{1}^{\prime} \cdot \mathrm{C}_{\mathrm{Cl}_{2}}-\mathrm{k}_{2}^{\prime} \frac{\mathrm{C}_{\mathrm{HC1}}^{4} \cdot \mathrm{C}_{\mathrm{O}_{2}}}{\mathrm{C}_{\mathrm{Cl}_{2}} \cdot \mathrm{C}_{\mathrm{H}_{2} \mathrm{O}}^{2}}
$$

Combining Equations (11), (12), (13), (14) and (56) gives

$$
\begin{aligned}
& \left(-\mathrm{r}_{\mathrm{Cl}_{2}}\right)=\mathrm{k}_{1}^{\prime} \cdot \frac{{ }_{\mathrm{C} 1_{2}}^{\mathrm{O}}\left(\mathrm{N}_{1}+1\right)\left(1-\mathrm{x}_{\mathrm{Cl}}\right)}{2\left(\mathrm{~N}_{1}+1\right)+\mathrm{x}_{\mathrm{C} 1_{2}}}
\end{aligned}
$$

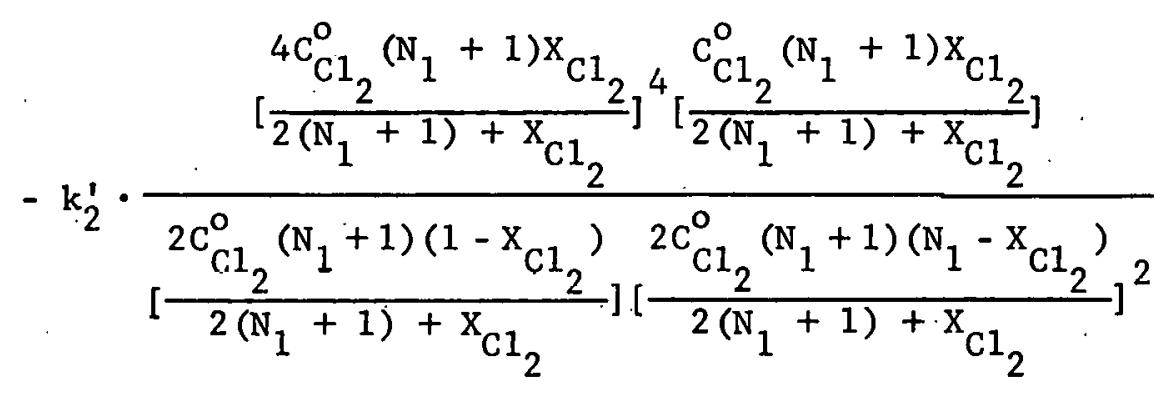

After rearranging and using Equation (49),

$$
\begin{aligned}
& \left(-\mathrm{r}_{\mathrm{Cl}}\right)=\frac{2 \mathrm{k}_{1}^{\prime} \mathrm{C}_{\mathrm{Cl}}^{\mathrm{O}}{ }^{\left(\mathrm{N}_{1}+1\right)}}{2\left(\mathrm{~N}_{1}+1\right)+\mathrm{x}_{\mathrm{Cl}}{ }_{2}} \\
& \left\{1-\mathrm{x}_{\mathrm{C} 1_{2}}-\frac{16 \cdot \mathrm{C}_{\mathrm{Cl}}^{\mathrm{o}}\left(\mathrm{N}_{1}+1\right) \mathrm{x}_{\mathrm{Cl}}^{5}}{\mathrm{~K}_{\mathrm{eq} \cdot}^{\prime} \cdot\left(\mathrm{N}_{1}-\mathrm{x}_{\mathrm{Cl}}\right)^{2} \cdot\left(1-\mathrm{x}_{\mathrm{Cl}}\right)\left[2\left(\mathrm{~N}_{1}+1\right)+\mathrm{x}_{\mathrm{Cl}}\right]}\right\}
\end{aligned}
$$


Combining Equations (27) and (58), and rearranging

$$
\begin{aligned}
& \tau=\frac{1}{2 k_{1}^{\prime}\left(N_{1}+1\right)} \int_{0}^{x_{C 1}^{f}}\left[2\left(N_{1}+1\right)+x_{C 1}\right]
\end{aligned}
$$

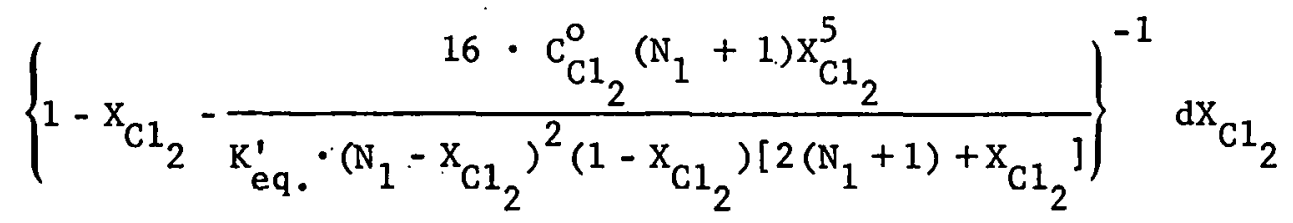

$$
\begin{aligned}
& \left\{1-\mathrm{x}_{\mathrm{Cl}{ }_{2}}-\frac{16 \cdot \mathrm{C}_{\mathrm{C} 1_{2}}^{\mathrm{o}}\left(\mathrm{N}_{1}+1\right) \mathrm{x}_{\mathrm{Cl}}^{5}}{\mathrm{k} ! \cdot\left(\mathrm{N}_{1}-\mathrm{x}_{\mathrm{Cl} 1_{2}}\right)^{2} \cdot\left(1-\mathrm{x}_{\mathrm{C} 1_{2}}\right)\left[2\left(\mathrm{~N}_{1}+1\right)+\mathrm{x}_{\mathrm{Cl}}\right]}\right\}^{-1} \mathrm{dx} \mathrm{Cl}_{2}
\end{aligned}
$$

Then

$$
\mathrm{X}=\mathbf{k}^{\prime} \tau
$$

2. If the forward reaction rate equation is

$$
\begin{aligned}
& \text { Rate }=\mathrm{k}_{1}^{\prime} \mathrm{C}_{\mathrm{Cl}_{2}}^{2} \mathrm{C}_{\mathrm{H}_{2} \mathrm{O}}: \\
& \text { Rate }=\left(-\mathrm{r}_{\mathrm{Cl}_{2}}\right)=\mathrm{k}_{1}^{\prime} \mathrm{C}_{\mathrm{Cl}_{2}}^{2} \mathrm{C}_{\mathrm{H}_{2} \mathrm{O}}-\frac{\mathrm{L}_{\mathrm{HCl} \mathrm{O}_{2}}^{4}}{\mathrm{C}_{\mathrm{H}_{2} \mathrm{O}}}
\end{aligned}
$$

Combining Equations (11), (12), (13), (14), and (62) gives

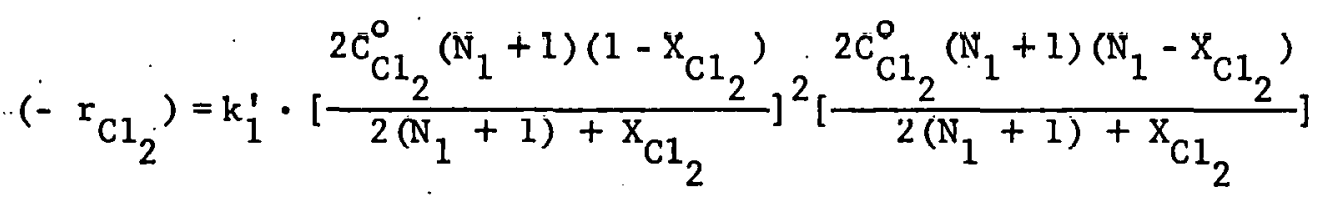




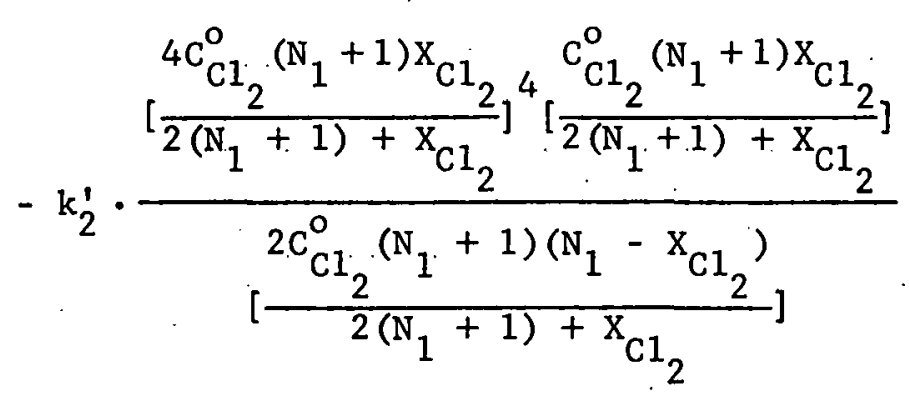

After rearranging and using Equation (49),

$$
\begin{aligned}
& \left(-\mathrm{r}_{\mathrm{Cl}_{2}}\right)=\frac{8 \mathrm{k}_{1}^{\prime}\left[\mathrm{C}_{\mathrm{Cl}}^{\mathrm{o}}\right]^{3}\left(\mathrm{~N}_{1}+1\right)^{3}}{\left[2\left(\mathrm{~N}_{1}+1\right)+\mathrm{X}_{\mathrm{Cl} 1_{2}}\right]^{3}}\left\{\left(1-\mathrm{X}_{\mathrm{Cl} 1_{2}}\right)^{2}\left(\mathrm{~N}_{1}-\mathrm{x}_{\mathrm{C} 1_{2}}\right)\right.
\end{aligned}
$$

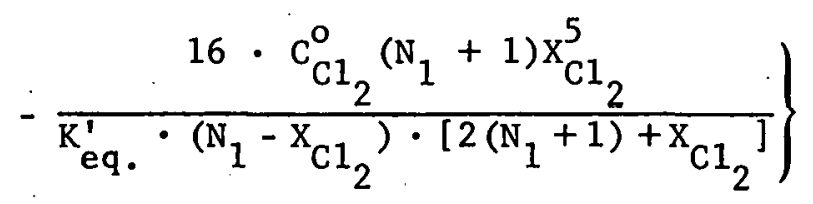

Combining Equations (27) and (64) and rearranging

$$
\begin{aligned}
& \tau=\frac{1}{8 \mathrm{k}_{1}^{\prime} \cdot\left[\mathrm{C}_{\mathrm{Cl}{ }_{2}}^{\mathrm{o}}\right]^{2} \cdot\left(\mathrm{N}_{1}+1\right)^{3}} \int_{0}^{\mathrm{X}_{\mathrm{C} 1_{2}}^{\mathrm{f}}}\left[2\left(\mathrm{~N}_{1}+1\right)+\mathrm{x}_{\mathrm{C} 1_{2}}\right]^{3} \\
& \left\{\left(1-\mathrm{x}_{\mathrm{Cl}_{2}}\right)^{2}\left(\mathrm{~N}_{1}-\mathrm{x}_{\mathrm{Cl}_{2}}\right)-\frac{16 \cdot \mathrm{C}_{\mathrm{Cl} 1_{2}}^{\mathrm{O}} \cdot\left(\mathrm{N}_{1}+1\right) \cdot \mathrm{x}_{\mathrm{Cl}_{2}}^{5}}{\mathrm{~K}_{\mathrm{eq} \cdot}^{\prime} \cdot\left(\mathrm{N}_{1}-\mathrm{x}_{\mathrm{Cl}}\right) \cdot\left[2\left(\mathrm{~N}_{1}+1\right)+\mathrm{x}_{\mathrm{Cl}_{2}}\right]}\right\}^{-1} \\
& \mathrm{dx}_{\mathrm{Cl}_{2}}
\end{aligned}
$$

Let $\quad \mathrm{X}=\frac{1}{8 \cdot\left[\mathrm{C}_{\mathrm{Cl}}\right]_{2}^{2} \cdot\left(\mathrm{N}_{1}+1\right)^{3}} \int_{0}^{\mathrm{X}_{\mathrm{Cl}}^{\mathrm{f}}}\left[2\left(\mathrm{~N}_{1}+1\right)+\mathrm{X}_{\mathrm{Cl}}\right]^{3}$.

$$
\left\{\left(1-\mathrm{x}_{\mathrm{Cl}}\right)^{2}\left(\mathrm{~N}_{1}-\mathrm{x}_{\mathrm{Cl}}\right)-\frac{\left.16 \cdot \mathrm{c}_{2}\right)-\left(\mathrm{N}_{2}+1\right) \cdot \mathrm{x}_{\mathrm{Cl}}^{5}}{\mathrm{~K}_{\mathrm{eq}}^{\prime} \cdot\left(\mathrm{N}_{1}-\mathrm{x}_{\mathrm{Cl}}\right)\left[2\left(\mathrm{~N}_{1}+1\right)+\mathrm{x}_{\mathrm{Cl}}\right]}\right\}^{-1} \mathrm{dx}_{\mathrm{Cl}_{2}}
$$


Then

$$
\mathrm{X}=\mathrm{k}_{1}^{\prime} \tau
$$

A plot of $X$ versus $\tau$ will give a straight line of $s$ lope $k_{1}$ or slope $k_{1}^{\prime}$ as shown in Equations (55), (61), and (67), if the correct rate expression has been chosen. The value of $\mathrm{X}$ in Equations (54), (60), and (66) can only be obtained by using a numerical integration method. Simpson's method was used in those cases.

The Values of $\mathrm{K}_{\mathrm{eq}}$. and $\mathrm{K}_{\mathrm{eq}}^{\prime}$.

The values of $\mathrm{K}_{\mathrm{eq}}$. and $\mathrm{K}_{\mathrm{eq}}^{\prime}$. at both 900 and $950^{\circ} \mathrm{K}$ should be available for calculating the values of $X$ in Equations (55), (61), and (67). The equilibrium constant (based on activities) of the Deacon reaction is expressed as a function of temperature in Equation (1). A signchange for the right-hand side terms of Equation (1) yields Equation (68), in which the equilibrium constant of the reverse Deacon reaction (Reaction II) is expressed as a function of temperature.

$$
\begin{aligned}
& \log \mathrm{K}^{\prime}=-\frac{4881.7}{\mathrm{~T}}+0.93035 \log \mathrm{T}-\left(1.3704 \times 10^{-4}\right) \mathrm{T} \\
& \quad+\left(1.7581 \times 10^{-8}\right) \mathrm{T}^{2}+4.1744 \\
& \mathrm{~K}^{\prime}=\frac{\left(\mathrm{a}_{\mathrm{HCl}}\right)^{4}\left(\mathrm{a}_{\mathrm{O}_{2}}\right)}{\left(\mathrm{a}_{\mathrm{Cl}}\right)^{2}\left(\mathrm{a}_{\mathrm{H}_{2} \mathrm{O}}\right)^{2}}
\end{aligned}
$$




$$
\begin{aligned}
& { }_{\mathrm{Cl}_{2}}=\mathrm{M}_{\mathrm{Cl}_{2}} \cdot \nu_{\mathrm{Cl}_{2}} \cdot \mathrm{P}_{\mathrm{t}} \\
& \mathrm{a}_{\mathrm{H}_{2} \mathrm{O}}=\mathrm{M}_{\mathrm{H}_{2} \mathrm{O}} \cdot \nu_{\mathrm{H}_{2} \mathrm{O}} \cdot \mathrm{P}_{\mathrm{t}} \\
& \mathrm{a}_{\mathrm{HCl}}=\mathrm{M}_{\mathrm{HCl}} \cdot \nu_{\mathrm{HCl}} \cdot \mathrm{P}_{\mathrm{t}} \\
& \mathrm{a}_{\mathrm{O}_{2}}=\mathrm{M}_{\mathrm{O}_{2}} \cdot \nu_{\mathrm{O}_{2}} \cdot \mathrm{P}_{\mathrm{t}}
\end{aligned}
$$

At atmospheric pressure the fugacity coefficients for all gases are close to unity. And $\mathrm{P}_{\mathrm{t}}=1$ atm., so Equation (70) becomes

$$
\begin{aligned}
& a_{\mathrm{Cl}_{2}}=\mathrm{M}_{\mathrm{Cl}_{2}}=\frac{{ }_{\mathrm{Cl}_{2}}}{\mathrm{n}_{\mathrm{t}}} \\
& \mathrm{a}_{\mathrm{H}_{2} \mathrm{O}}=\mathrm{M}_{\mathrm{H}_{2} \mathrm{O}}=\frac{\mathrm{n}_{2} \mathrm{O}}{\mathrm{n}_{\mathrm{t}}} \\
& \mathrm{a}_{\mathrm{HCl}}=\mathrm{M}_{\mathrm{HCl}}=\frac{\mathrm{n}_{\mathrm{HCl}}}{\mathrm{n}_{\mathrm{t}}} \\
& \mathrm{a}_{\mathrm{O}_{2}}=\mathrm{M}_{\mathrm{O}_{2}}=\frac{\mathrm{n}_{2}}{\mathrm{n}_{\mathrm{t}}}
\end{aligned}
$$

Combining Equations (69) and (71),

$$
K^{\prime}=\frac{\left(\frac{n_{H C 1}}{n_{t}}\right)^{4}\left(\frac{n_{2}}{n_{t}}\right)}{\left.\left.{ }_{n_{C 1}}\right)_{t}\right)^{2}\left(\frac{{ }_{H_{2}}}{n_{t}}\right)^{2}}=\frac{n_{H C 1}^{4} \cdot n_{O_{2}}}{n_{C 1}^{2} \cdot n_{H_{2} O}^{2}} \cdot \frac{1}{n_{t}}
$$

Because $\cdot \mathrm{C}_{\mathrm{Cl}_{2}}$, eq. $=\frac{\mathrm{n}_{\mathrm{C}{ }_{2}}}{\mathrm{~V}}$

$$
\left.\begin{array}{l}
\mathrm{C}_{\mathrm{H}_{2} \mathrm{O} \text {, eq. }}=\frac{\mathrm{n}_{2} \mathrm{O}}{\mathrm{v}} \\
\mathrm{C}_{\mathrm{HCl} \text {, eq. }}=\frac{\mathrm{n}_{\mathrm{IICl}}}{\mathrm{V}} \\
\mathrm{C}_{\mathrm{O}_{2}, \text { eq. }}=\frac{\mathrm{n}_{2}}{\mathrm{~V}}
\end{array}\right\}
$$


Equation (72) can be rearranged and combined with Equation (73)

$$
K^{\prime}=\frac{\left(\mathrm{C}_{\mathrm{HC} 1, \text { eq. }}\right)^{4}\left(\mathrm{C}_{\mathrm{O}_{2}, \text { eq. }}\right)}{\left(\mathrm{C}_{\mathrm{Cl} \mathrm{I}_{2}, \text { eq. }}\right)^{2}\left(\mathrm{C}_{\mathrm{H}_{2} \text { o, eq. }}\right)^{2}} \cdot \frac{\mathrm{v}}{\mathrm{n}_{\mathrm{t}}}
$$

Combining Equations (49) and (74) gives

$$
K_{e q .}^{\prime}=K^{\prime} \times \frac{n_{t}}{V}
$$

1. For Reaction II

$\mathrm{K}^{\prime}$, calculated from Equation (68), equals 1.899 at $900^{\circ} \mathrm{K}$ and 4.356 at $950^{\circ} \mathrm{K}$. Using the ideal gas law, $\mathrm{n}_{\mathrm{t}} / \mathrm{V}$ equals $0.01355 \mathrm{~g}$-moles/l at $900^{\circ} \mathrm{K}$ and $0.1284 \mathrm{~g}$-moles $/ \mathrm{l}$ at $950^{\circ} \mathrm{K}$. Therefore, $\mathrm{K}_{\mathrm{eq}}^{\prime}$.' calculated from Equation (75), equals 0.0257 at $900^{\circ} \mathrm{K}$ and 0.0559 at $950^{\circ} \mathrm{K}$.

\section{For Reaction I}

The equilibrium constant $K$, based on activity, can be expressed as

$$
\frac{\left(a_{\mathrm{HCl}}\right)^{2} \cdot\left(\mathrm{a}_{\mathrm{O}_{2}}\right)^{1 / 2}}{{ }^{\mathrm{a}} \mathrm{Cl}_{2} \cdot \mathrm{a}_{\mathrm{H}_{2} \mathrm{O}}}
$$

By comparing this expression with Equation (69), the following equation can be derived.

$$
K=\left(K^{\prime}\right)^{1 / 2}
$$

Combining Equations (71) and (73) with the activity-based expression of $\mathrm{K}$, 


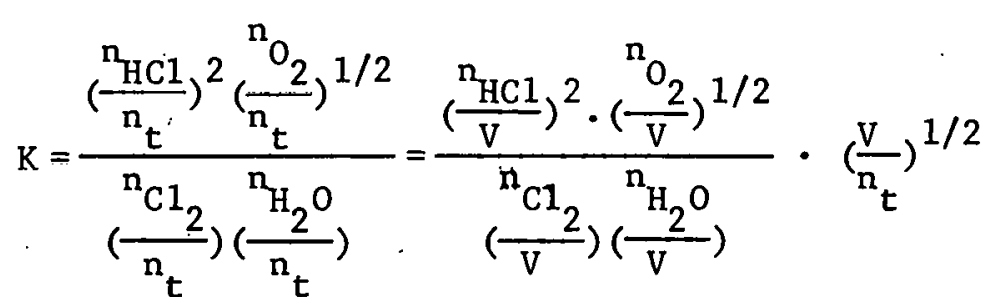

$$
\begin{aligned}
& =\frac{\left(\mathrm{C}_{\mathrm{HC} 1, \text { eq. }}\right)^{2} \cdot\left(\mathrm{C}_{\mathrm{O}_{2}, \text { eq. } .}\right)^{1 / 2}}{\left(\mathrm{C}_{\mathrm{C} 1} \text {, eq }\right)\left(\mathrm{C}_{\mathrm{H}_{2} \mathrm{O}, \text { eq. }}\right)} \cdot\left(\frac{\mathrm{V}}{\mathrm{n}_{\mathrm{t}}}\right)^{1 / 2}
\end{aligned}
$$

Combining Equations (48) and (77) gives

$$
\mathrm{K}_{\mathrm{eq} .}=\mathrm{K} \times\left(\frac{\mathrm{n}}{\mathrm{v}}\right)^{1 / 2}
$$

$\mathrm{K}$, calculated from Equation (76), equals 1.378 at $900^{\circ} \mathrm{K}$ and 2.087 at $950^{\circ} \mathrm{K}$. $\mathrm{K}_{\text {eq. }}$ calculated from Equation (78), equals 0.1604 at $9000 \mathrm{~K}$ and 0.2365 at $950^{\circ} \mathrm{K}$. 
RESULTS AND DISCUSSION

The Results and Discussion of Five-Pass Reactor at 900 and $950^{\circ} \mathrm{K}$

The experiments were run at atmospheric pressure and two temperatures, 900 and $950^{\circ} \mathrm{K}$. The results are given in Tables 1 and 2 . The theoretical equilibrium conversions are also listed in the table. Since the measured conversions are always less than $50 \%$ of the equilibrium conversions, the reverse reaction is considered negligible. Later, the results calculated by including the reverse reaction will be presented to show that the reverse reaction can actually be neglected.

Table 3 lists the twelve kinetic models which were tested for the forward reaction.

Appendix III explains why models $G, H$, and $L$ are suggested. Among the twelve models in Table 3 , model $\mathrm{B}$ appears to give the best fit of the results. A plot of Equation (44) is shown in Figure 5 for both 900 and $950^{\circ} \mathrm{K}$ experiments. Table 4 lists the values of $\mathrm{X}$ calculated from Equation (43). The values of $k_{1}$ are $0.032 \mathrm{sec}^{-1}$ at $900^{\circ} \mathrm{K}$ and $0.051 \mathrm{sec}^{-1}$ at $950^{\circ} \mathrm{K}$. Based on the Arrhenius relationship, $k=A \cdot \exp ,(-E / R T)$, gives $A=$ frequency factor $=224 \sec ^{-1}$ and $E=$ activation energy $=66.2 \mathrm{~kJ} / \mathrm{g}$-mole. (See Appendix IV.)

Al1 twelve rate expressions were similarly analyzed. With one exception, model $\mathrm{E}$, none of the other rate expressions approximated a linear, relationship (e.g. Figures 6 and 7 of model $C$ and Figures 8 and 9 of model L, Table 4 lists the values of $X$ for these two models). A plot of Equation (47) is shown in Figure 10 for both 900 and $9500 \mathrm{~K}$ experiments. Table 4 lists the values of X calculated from Equation (46). 
Table 1. Experimental results of five-pass reactor at $900^{\circ} \mathrm{K}^{2}$

\begin{tabular}{|c|c|c|c|c|c|}
\hline $\begin{array}{c}\text { Run } \\
\text { number }\end{array}$ & $\begin{array}{c}\mathrm{U}_{\mathrm{C} 1_{2}}^{\mathrm{b}} \\
\text { (cc/min.) }\end{array}$ & $\begin{array}{c}\mathrm{U}_{\mathrm{H}_{2} \mathrm{O}}^{\mathrm{O}} \mathrm{b} \\
\text { (cc/min.) }\end{array}$ & $\mathrm{U}^{\mathrm{o}^{\mathrm{b}}}$ & $\begin{array}{c}\tau \\
\left(\sec ^{2}\right)\end{array}$ & $\begin{array}{l}\mathrm{C}_{\mathrm{C} \mathrm{I}_{2}}^{\mathrm{o}} \times 10^{3} \\
(\mathrm{~g} \text {-moles } / \ell)\end{array}$ \\
\hline $\mathrm{A} 1$ & 364 & 483 & 847 & 2.41 & 5.823 \\
\hline A2 & 242 & 483 & 725 & 2.81 & 4.523 \\
\hline A3 & 364 & 483 & 847 & 2.41 & 5.823 \\
\hline A4 & 242 & 483 & 725 & 2.81 & 4.523 \\
\hline A5 & 266 & 284 & .550 & 3.71 & 6.559 \\
\hline A6 & 327 & 284 & 611 & 3.34 & 7.258 \\
\hline A7 & 266 & 284 & 550 & 3.71 & 6.559 \\
\hline A8 & 327 & 284 & 611 & 3.34 & 7.258 \\
\hline A9 & 364 & 416 & 780 & 2.62 & 6.327 \\
\hline A10 & 242 & 416 & 658 & 3.10 & 4.987 \\
\hline All & 364 & 416 & 780 & 2.62 & 6.327 \\
\hline A12 & 242 & 416 & 658 & 3.10 & 4.987 \\
\hline A13 & 15.5 & 308 & 463 & 4.41 & 4.523 \\
\hline A14 & 187 & 308 & 495 & 4.12 & 5.108 \\
\hline A15 & 224 & 308 & 532 & 3.84 & 5.691 \\
\hline A16 & 266 & 308 & 574 & 3.55 & 6.276 \\
\hline A17 & 328 & 308 & 636 & 3.21 & 6.976 \\
\hline A18 & 242 & 308 & 550 & 3.71 & 5.958 \\
\hline A19 & 155 & 316 & 471 & 4.33 & 4.450 \\
\hline A20 & 187 & 316 & 503 & 4.06 & 5.030 \\
\hline A21 & 223 & 316 & 539 & 3.78 & 5.610 \\
\hline A22 & 266 & 316 & 582 & 3.50 & 6.193 \\
\hline A23 & 327 & 316 & 643 & 3.17 & 6.893 \\
\hline A24 & 155 & 158 & 313 & 6.52 & 6.695 \\
\hline A25 & 187 & 158 & 345 & 5.92 & 7.333 \\
\hline A26 & 224 & 158 & 382 & 5.35 & 7.931 \\
\hline A27 & 266 & 158 & 424 & 4.81 & 8.498 \\
\hline A2 8 & 328 & 158 & 486 & 4.20 & 9.135 \\
\hline
\end{tabular}


Table 1. Continued

\begin{tabular}{|c|c|c|c|c|c|}
\hline $\begin{array}{c}\text { Run } \\
\text { number }\end{array}$ & $\begin{array}{l}\mathrm{C}_{\mathrm{H}_{2} \mathrm{O}}^{\mathrm{o}} \times 10^{3} \\
(\mathrm{~g} \text {-moles } / \ell)\end{array}$ & $\begin{array}{c}\mathrm{U}_{\mathrm{O}_{2}}{ }^{\mathrm{c}} \\
\text { (cc/min.) }\end{array}$ & $\mathrm{N}_{1}$ & $\begin{array}{l}\mathrm{X}_{\mathrm{C} 1_{2}}^{\mathrm{f}} \\
(\%)\end{array}$ & $\begin{array}{c}\left(\mathrm{x}_{\mathrm{C} 1_{2}}^{\mathrm{f}}, \mathrm{eq}\right. \\
(\%)\end{array}$ \\
\hline $\mathrm{Al}$ & 7.727 & 4.12 & 1.327 & 6.91 & 57.50 \\
\hline A2 & 9.027 & 3.12 & 1.996 & 7.88 & 68.00 \\
\hline A3 & 7.727 & 4.12 & 1.327 & 6.91 & 57.50 \\
\hline A4 & 9.027 & 2.80 & 1.976 & 7.06 & 68.00 \\
\hline A5 & 6.991 & 4.05 & 1.066 & 9.24 & 52.00 \\
\hline A6 & 6.292 & 4.38 & 0.867 & 8.16 & 47.00 \\
\hline A7 & 6.991 & 4.05 & 1.066 & 9.24 & 52.00 \\
\hline A8 & 6.292 & 4.40 & 0.867 & 8.20 & 47.00 \\
\hline A9 & 7.223 & 4.98 & 1.141 & 8.34 & 53.50 \\
\hline A10 & 8.563 & 4.27 & 1.717 & 10.77 & 64.00 \\
\hline Al1 & 7.223 & 4.96 & 1.141 & 8.31 & 53.50 \\
\hline A12 & 8.563 & 4.20 & 1.717 & 10.59 & 64.00 \\
\hline A.13 & 9.028 & 3.50 & 1.996 & 13.82 & 68.00 \\
\hline A14 & 8.442 & 4.37 & 1.653 & 14.29 & 63.00 \\
\hline A15 & 7.859 & 5.17 & 1.381 & 14.13 & 58.50 \\
\hline A16 & 7.274 & 5.93 & 1.159 & 13.60 & 54.00 \\
\hline A17 & 6.574 & 6.47 & 0.942 & 12.06 & 50.00 \\
\hline A18 & 7.592 & 5.40 & 1.274 & 13.62 & 56.50 \\
\hline A19 & 9.102 & 2.40 & 2.046 & 9.48 & 68.50 \\
\hline A20 & 8.520 & 3.63 & 1.694 & 11.87 & 63.50 \\
\hline A21 & 7.941 & 4.10 & 1.416 & 11.20 & 59.00 \\
\hline A22 & 7.357 & 5.40 & 1.188 & 12.38 & 55.00 \\
\hline A23 & 6.657 & 5.80 & 0.966 & 10.81 & 49.50 \\
\hline A24 & 6.855 & 4.43 & 1.024 & 17.50 & 51.00 \\
\hline A25 & 6.217 & 5.17 & 0.848 & 16.90 & 46.00 \\
\hline A26 & 5.619 & 5.40 & 0.708 & 14.76 & 42.00 \\
\hline A2 27 & 5.052 & 5.87 & 0.594 & 13.46 & 38.00 \\
\hline A28 & 4.415 & 6.50 & 0,483 & 12.12 & 33.00 \\
\hline
\end{tabular}

$\mathrm{c}_{\text {The rates }}$ are based on $295^{\circ} \mathrm{K}$. 
Table 2. Experimental results of five-pass reactor at $950 \mathrm{~K}^{\mathrm{a}}$

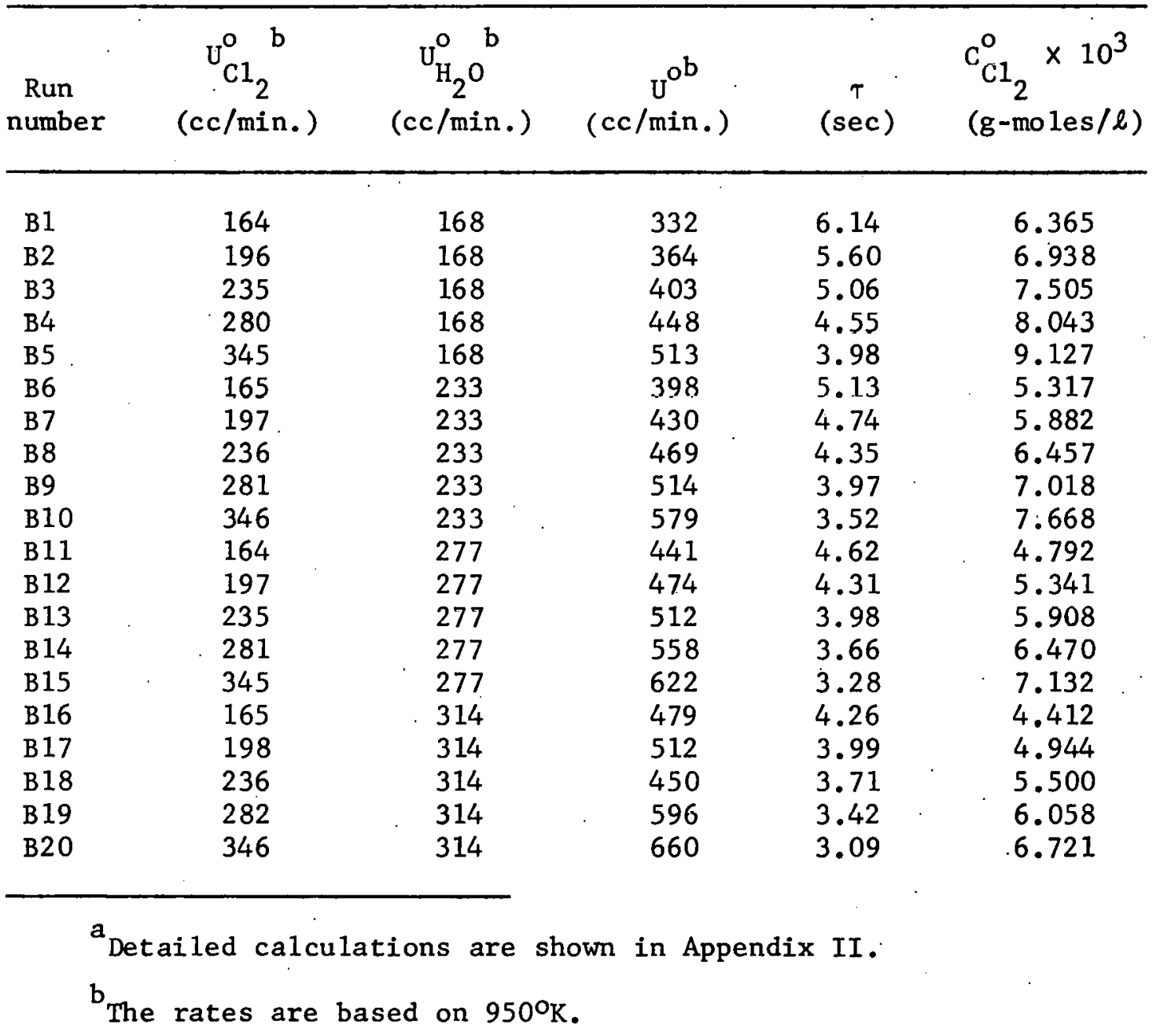


Table 2. Continued

\begin{tabular}{|c|c|c|c|c|c|}
\hline $\begin{array}{l}\text { Run } \\
\text { number }\end{array}$ & $\begin{array}{l}\mathrm{C}_{\mathrm{H}_{2} \mathrm{O}}^{\mathrm{O}} \times 10^{3} \\
(\mathrm{~g} \text {-moles/l) }\end{array}$ & $\begin{array}{c}\mathrm{U}_{\mathrm{O}_{2}}{ }^{\mathrm{c}} \\
\text { (cc/min.) }\end{array}$ & $\mathrm{N}_{1}$ & $\begin{array}{l}\mathrm{x}_{\mathrm{C} 1_{2}}^{\mathrm{f}} \\
(\%)\end{array}$ & $\left.\underset{\substack{\mathrm{Cl}_{2}, \mathrm{eq} \cdot \\
(\%)}}{\mathrm{f}}\right)$ \\
\hline $\mathrm{B} 1$ & 6.472 & 5.60 & 1.017 & 21.96 & 55.50 \\
\hline B2 & 5.898 & 7.10 & 0.850 & 23.28 & 57.50 \\
\hline B3 & 5.332 & 8.76 & 0.710 & 24.00 & 53.00 \\
\hline B4 & 4.794 & 10.07 & 0.596 & 23.15 & 46.00 \\
\hline B5 & 4.423 & 11.50 & 0.485 & 21.50 & 40.00 \\
\hline $\mathrm{B} 6$ & 7.520 & 5.00 & 1.414 & 19.61 & 73.50 \\
\hline B7 & 6.955 & 6.20 & 1.182 & 20.33 & 68.00 \\
\hline B8 & 6.380 & 7.50 & 0.988 & 20.55 & 62.50 \\
\hline B9 & 5.819 & 8.80 & 0.829 & 20.23 & 56.50 \\
\hline $\mathrm{B} 10$ & 5.169 & 9.60 & 0.674 & 17.94 & 50.00 \\
\hline B 11 & 8.044 & 4.77 & 1.678 & 18.69 & 77.50 \\
\hline B12 & 7.496 & 6.17 & 1.403 & 20.22 & 73.00 \\
\hline B13 & 6.928 & 6.77 & 1.173 & 18.54 & 67.50 \\
\hline B14 & 6.366 & 7.50 & 0.984 & 17.24 & 62.50 \\
\hline B 15 & 5.705 & 8.37 & 0.800 & 15.64 & 55.50 \\
\hline B16 & 8.424 & 5.07 & 1.909 & 19.87 & 81.00 \\
\hline B 17 & 7.892 & 6.07 & 1.596 & 19.89 & 76.00 \\
\hline B 18 & 7.336 & 7.03 & 1.334 & 19.27 & 72.00 \\
\hline B 19 & 6.779 & 8.07 & 1.119 & 18.54 & 66.00 \\
\hline B20 & 6.116 & 8.63 & 0.910 & 16.14 & 60.00 \\
\hline
\end{tabular}

$c_{\text {The rates are based on }} 295^{\circ} \mathrm{K}$. 
Table 3. Kinetic models

Mode1

Forward reaction rate expression

A

$\left(-\mathrm{r}_{\mathrm{Cl}_{2}}\right)=\mathrm{kC}_{\mathrm{Cl}_{2}} \mathrm{C}_{\mathrm{H}_{2} \mathrm{O}}$

B

$\left(-\mathrm{r}_{\mathrm{Cl}}\right)=\mathrm{kC}_{\mathrm{Cl}}$

C

$\left(-\mathrm{r}_{\mathrm{Cl}_{2}}\right)=\mathrm{kC}_{\mathrm{H}_{2} \mathrm{O}}$

D

$\left(-\mathrm{r}_{\mathrm{Cl}_{2}}\right)=\mathrm{k}$

E

$\left(-\mathrm{r}_{\mathrm{Cl}_{2}}\right)=\mathrm{kC}_{\mathrm{Cl}_{2}}^{2} \mathrm{C}_{\mathrm{H}_{2} \mathrm{O}}$

$\left(-\mathrm{r}_{\mathrm{Cl}_{2}}\right)={ }^{\mathrm{kC}} \mathrm{Cl}_{2} \mathrm{C}_{\mathrm{H}_{2} \mathrm{O}}^{2}$

$\left(-\mathrm{r}_{\mathrm{Cl}_{2}}\right)=k \frac{{ }_{\mathrm{Cl}_{2}}{ }^{\mathrm{C}_{\mathrm{H}_{2} \mathrm{O}}}}{\mathrm{C}_{\mathrm{HCl} 1}}$

$\mathrm{H}$

$\left(-\mathrm{r}_{\mathrm{Cl}{ }_{2}}\right)=\mathrm{k} \frac{\mathrm{C}_{\mathrm{Cl}{ }_{2}}^{2} \mathrm{C}_{\mathrm{H}_{2} \mathrm{O}}^{2}}{\mathrm{C}_{\mathrm{HC} 1}^{2}}$

I.

$\left(-\mathrm{r}_{\mathrm{Cl} 1_{2}}\right)=\mathrm{kC}_{\mathrm{Cl} 1_{2}}^{2} \mathrm{C}_{\mathrm{H}_{2} \mathrm{O}}^{2}$

$\mathrm{J}$

$\left(-\mathrm{r}_{\mathrm{Cl}_{2}}\right)=\mathrm{kC}_{\mathrm{Cl}_{2}}^{2}$

K,

$\left(-\mathrm{r}_{\mathrm{Cl}_{2}}\right)=\mathrm{kC}_{\mathrm{H}_{2} \mathrm{O}}^{2}$

L

$\left(-\mathrm{r}_{\mathrm{Cl}_{2}}\right)={ }^{\mathrm{kC}} \mathrm{Cl}_{2}^{1 / 2} \mathrm{C}_{\mathrm{H}_{2} \mathrm{O}}$ 


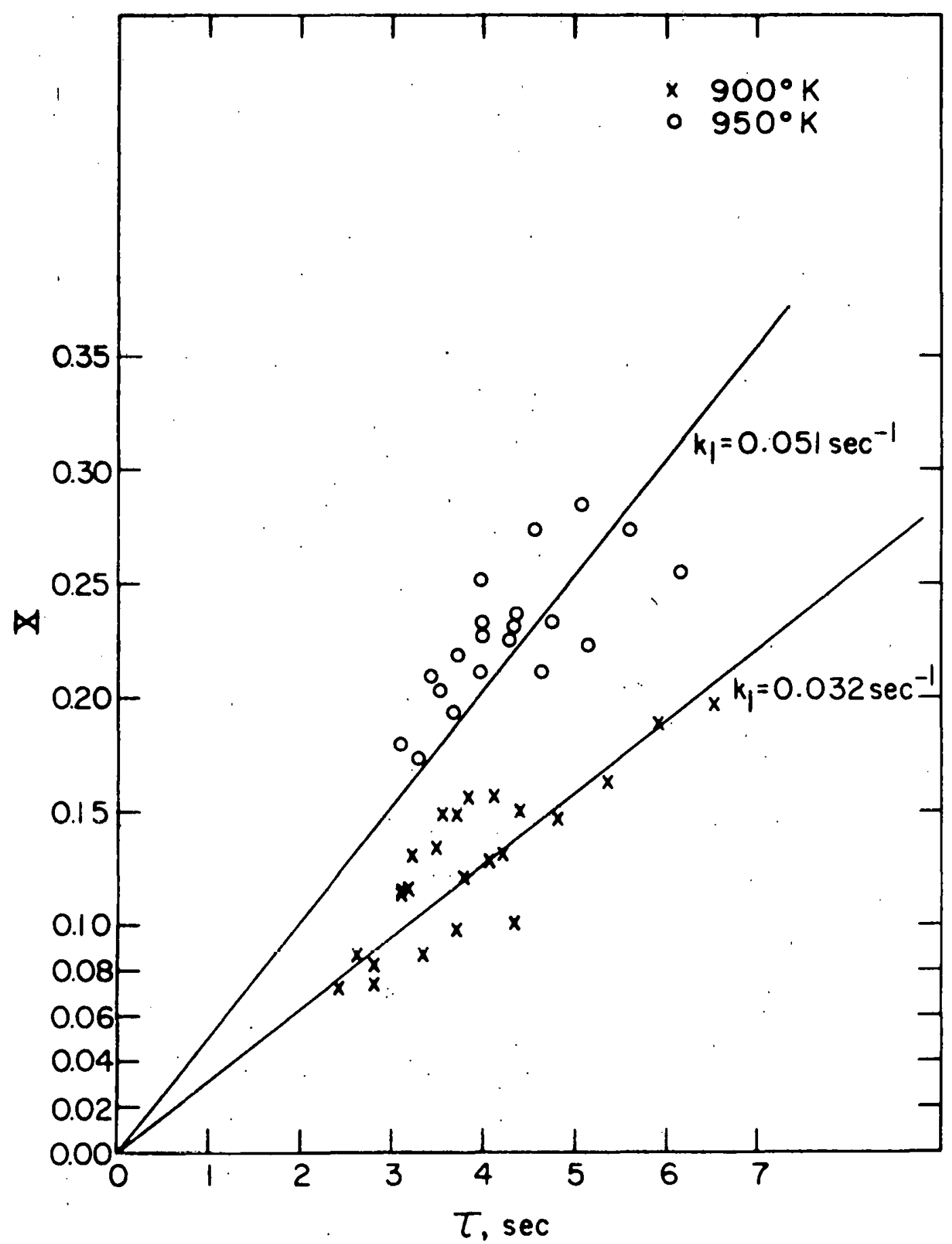

Figure 5. $\mathrm{X}$ as a function of space-time for model $\mathrm{B}$ (five-pass reactor) 
Table 4. Values of $\mathrm{X}$ Rumber Equation (43) Equation (54) Equation (60) Equation (46) Equation (66) Mode1 $\mathrm{C}^{\mathrm{a}} \cdot$ Model $^{\mathrm{b}}$

\begin{tabular}{llllllll}
\hline A1 & 0.0721 & 0.0721 & 0.0722 & 1736 & 1710 & 0.0539 & 2.041 \\
A2 & 0.0826 & 0.0826 & 0.0826 & 2182 & 2152 & 0.0406 & 1.746 \\
A3 & 0.0721 & 0.0721 & 0.0722 & 1736 & 1710 & 0.0539 & 2.041 \\
A4 & 0.0736 & 0.0736 & 0.0737 & 1930 & 1902 & 0.0362 & 1.556 \\
A5 & 0.0980 & 0.0980 & 0.0982 & 2409 & 2377 & 0.0917 & 3.300 \\
A6 & 0.0861 & 0.0861 & 0.0862 & 2118 & 2089 & 0.0999 & 3.409 \\
A7 & 0.0980 & 0.0980 & 0.0982 & 2918 & 2377 & 0.0917 & 3.300 \\
A8 & 0.0865 & 0.0865 & 0.0866 & 2129 & 2101 & 0.1005 & 3.427 \\
A9 & 0.0379 & 0.0879 & 0.0880 & 2132 & 2106 & 0.0766 & 2.800 \\
A10 & 0.1151 & 0.1151 & 0.1152 & 3011 & 2975 & 0.0654 & 2.710 \\
A11 & 0.0376 & 0.0876 & 0.0877 & 2124 & 2097 & 0.0764 & 2.789 \\
A12 & 0.1130 & 0.1130 & 0.1131 & 2951 & 2916 & 0.0643 & 2.661 \\
A13 & 0.1505 & 0.1505 & 0.1507 & 4235 & 4177 & 0.0726 & 3.187 \\
A14 & 0.1563 & 0.1563 & 0.1567 & 4228 & 4178 & 0.0916 & 3.796 \\
A15 & 0.1546 & 0.1546 & 0.1551 & 4081 & 4032 & 0.1096 & 4.301 \\
A16 & 0.1485 & 0.1485 & 0.1490 & 3816 & 3822 & 0.1268 & 4.736 \\
A17 & 0.1306 & 0.1306 & 0.1309 & 3369 & 3332 & 0.1392 & 4.907 \\
A18 & 0.1486 & 0.1486 & 0.1490 & 3876 & 3832 & 0.1148 & 4.398 \\
A19 & 0.1004 & 0.1004 & 0.1004 & 2714 & 2678 & 0.0478 & 2.087 \\
A20 & 0.1278 & 0.1278 & 0.1279 & 3378 & 3335 & 0.0735 & 3.041 \\
& & & & & &
\end{tabular}

$$
\begin{aligned}
& a_{x}=\frac{-x_{C 1_{2}}^{f}-\left(3 N_{1}+2\right) \ln \left(\left(N_{1}-x_{C 1_{2}}^{f}\right) / N_{1}\right)}{2\left(N_{1}+1\right)}
\end{aligned}
$$

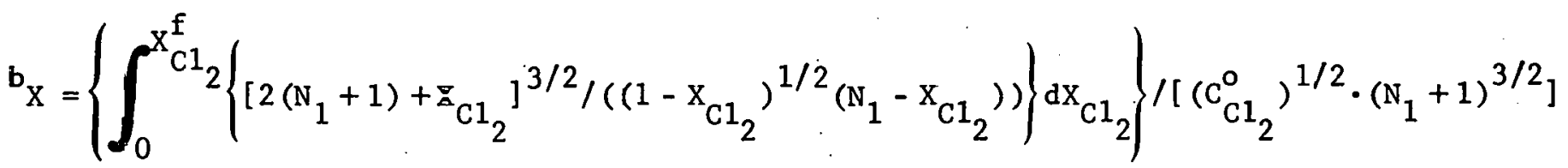


Table 4. Continued

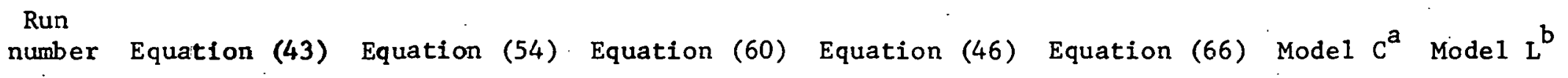

\begin{tabular}{|c|c|c|c|c|c|c|c|}
\hline A21 & 0.1202 & 0.1202 & 0.1203 & 3062 & 3024 & 0.0834 & 3.263 \\
\hline $\mathrm{A} 22$ & 0.1341 & 0.1341 & 0.1344 & 3438 & 3391 & 0.1116 & 4.178 \\
\hline A23 & 0.1160 & 0.1160 & 0.1162 & 2934 & 2895 & 0.1203 & 4.250 \\
\hline A24 & 0.1967 & 0.1967 & 0.1981 & 5480 & 5413 & 0.1916 & 7.032 \\
\hline A25 & 0.1895 & 0.1895 & 0.1911 & 5402 & 5340 & 0.2275 & 7.974 \\
\hline A26 & 0.1632 & 0.1633 & 0.1645 & 4708 & 4656 & 0.2390 & 7.999 \\
\hline A27 & 0.1477 & 0.1477 & 0.1488 & 4429 & 4383 & 0.2626 & 8.457 \\
\hline A28 & 0.1319 & 0.1319 & 0.1329 & 4241 & 4190 & 0.2951 & 9.129 \\
\hline B1 & 0.2550 & 0.2550 & 0.2574 & 8511 & 8430 & 0.2502 & 9.582 \\
\hline B2 & 0.2737 & 0.2738 & 0.2779 & 9779 & 9695 & 0.3306 & 12.224 \\
\hline B3 & 0.2845 & 0.2848 & 0.2909 & 11078 & 11000 & 0.4280 & 15.300 \\
\hline B4 & 0.2733 & 0.2736 & 0.2806 & 11456 & 11385 & 0.5110 & 17.626 \\
\hline B5 & 0.2512 & 0.2516 & 0.2589 & 10405 & 10347 & 0.6090 & 19.634 \\
\hline B6 & 0.2229 & 0.2229 & 0.2238 & 7058 & 6982 & 0.1524 & 6.311 \\
\hline B7 & 0.2328 & 0.2328 & 0.2342 & 7446 & 7370 & 0.1933 & 7.641 \\
\hline B 8 & 0.2362 & 0.2362 & 0.2382 & .7740 & 7663 & 0.2394 & 9.059 \\
\hline B9 & 0.2325 & 0.2326 & 0.2349 & 7890 & 7816 & 0.2878 & 10.451 \\
\hline B10 & 0.2032 & 0.2032 & 0.2051 & 7085 & 7014 & 0.3182 & 10.972 \\
\hline B11 & 0.2106 & 0.2106 & 0.2112 & 6720 & 6645 & 0.1202 & 5.218 \\
\hline B12 & 0.2308 & 0.2308 & 0.2319 & 7369 & 7292 & 0.1590 & 6.584 \\
\hline B13 & 0.2096 & 0.2096 & 0.2106 & 6526 & 6456 & 0.1758 & 6.889 \\
\hline B14 & 0.1935 & 0.1935 & 0.1944 & 6005 & 5940 & 0.1969 & 7.348 \\
\hline B15 & 0.1739 & 0.1739 & 0.1747 & 5475 & 5415 & 0.2224 & 7.869 \\
\hline B16 & 0.2254 & 0.2254 & 0.2261 & 7481 & 7396 & 0.1118 & 5.076 \\
\hline B 17 & 0.2262 & 0.2262 & 0.2270 & 7271 & 7193 & 0.1357 & 5.828 \\
\hline B18 & 0.2186 & 0.2186 & 0.2196 & 6874 & 6799 & 0.1593 & 6.481 \\
\hline B19 & 0.2097 & 0.2097 & 0.2108 & 6550 & 6480 & 0.1852 & 7.170 \\
\hline B20 & 0.1798 & 0.1798 & 0.1807 & 5552 & 5492 & 0.1995 & 7.278 \\
\hline
\end{tabular}




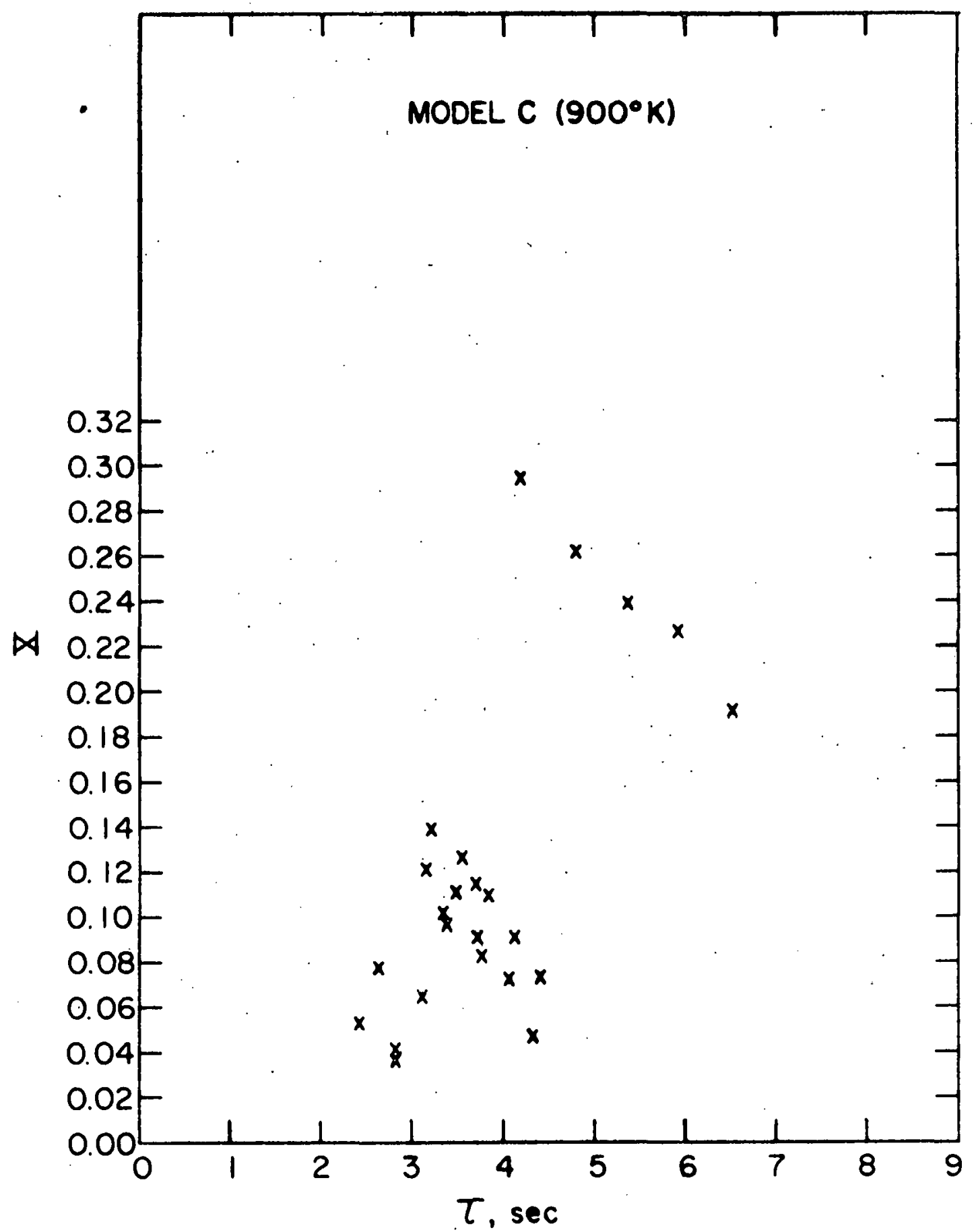

Figure 6. $\mathrm{X}$ as a function of space-time for model $\mathrm{C}$ at $900^{\circ} \mathrm{K}$ (five-pass reactor) 


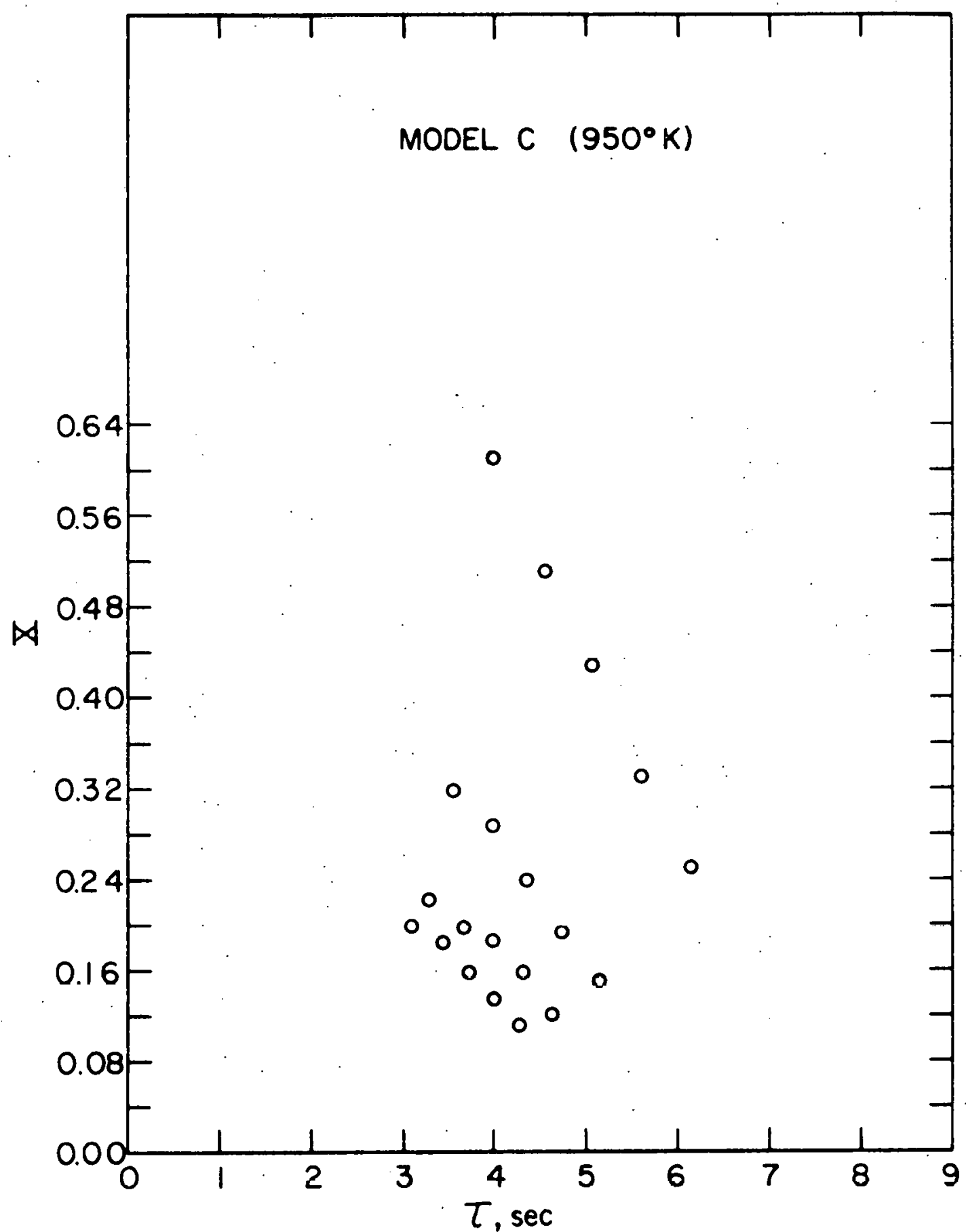

Figure 7. $\mathrm{X}$ as a function of space-time for model $\mathrm{C}$ at $950^{\circ} \mathrm{K}$ (five-pass reactor) 


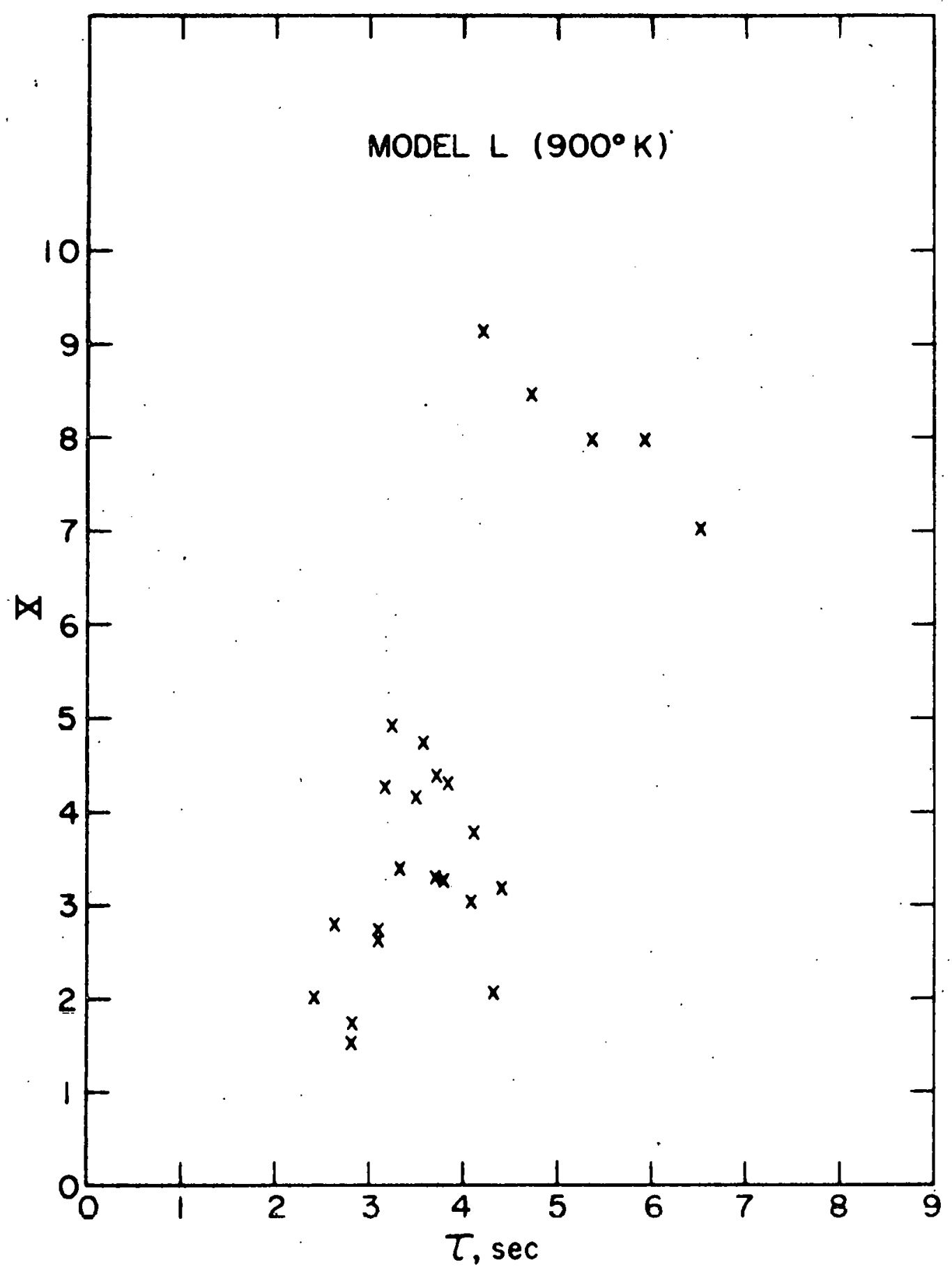

Figure 8. $X$ as a function of space-time for model $\mathrm{L}$ at $900^{\circ} \mathrm{K}$ (five-pass reactor) 


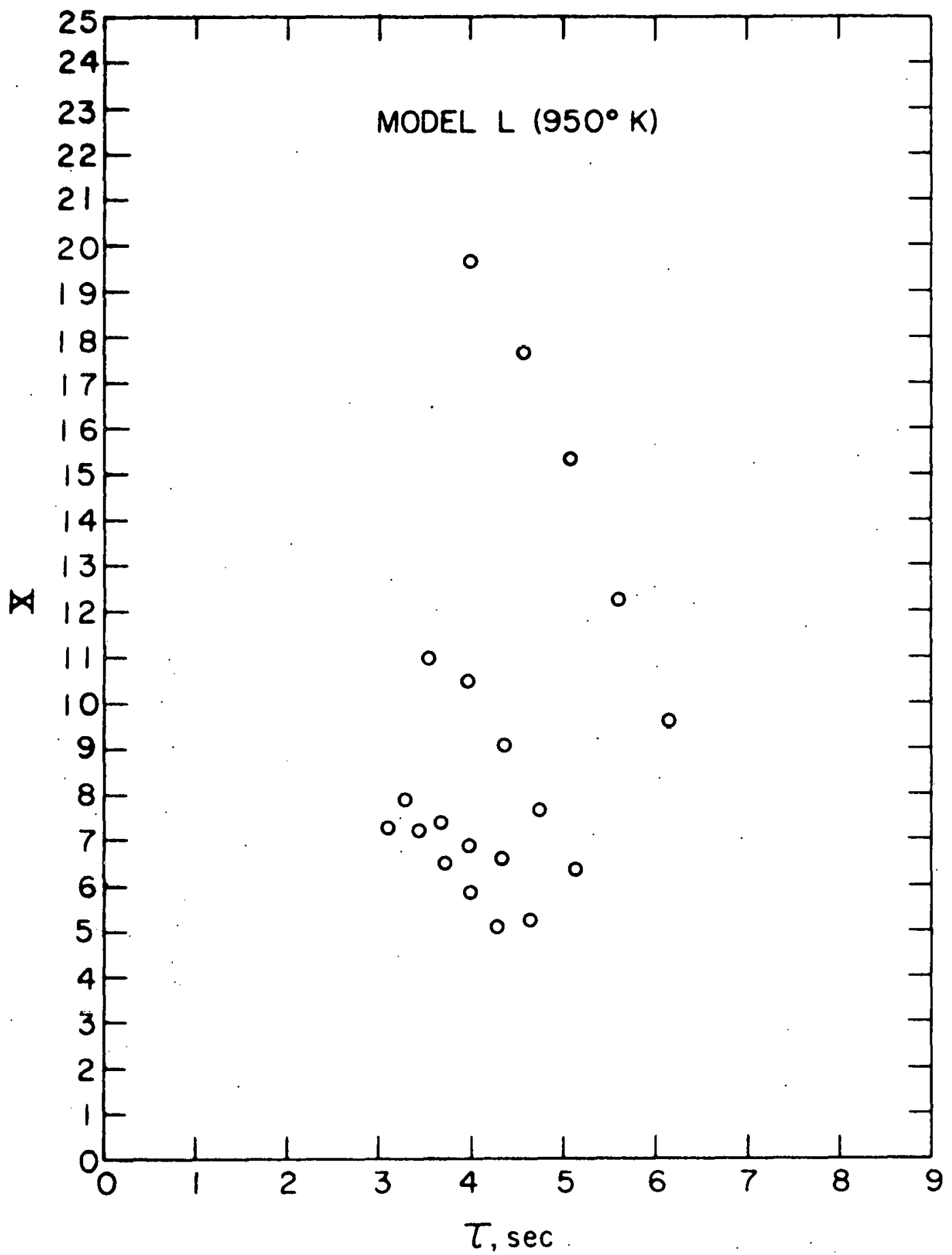

Figure 9. $\mathrm{X}$ as a function of space-time for model $\mathrm{L}$ at $950^{\circ} \mathrm{K}$ (five-pass reactor) 


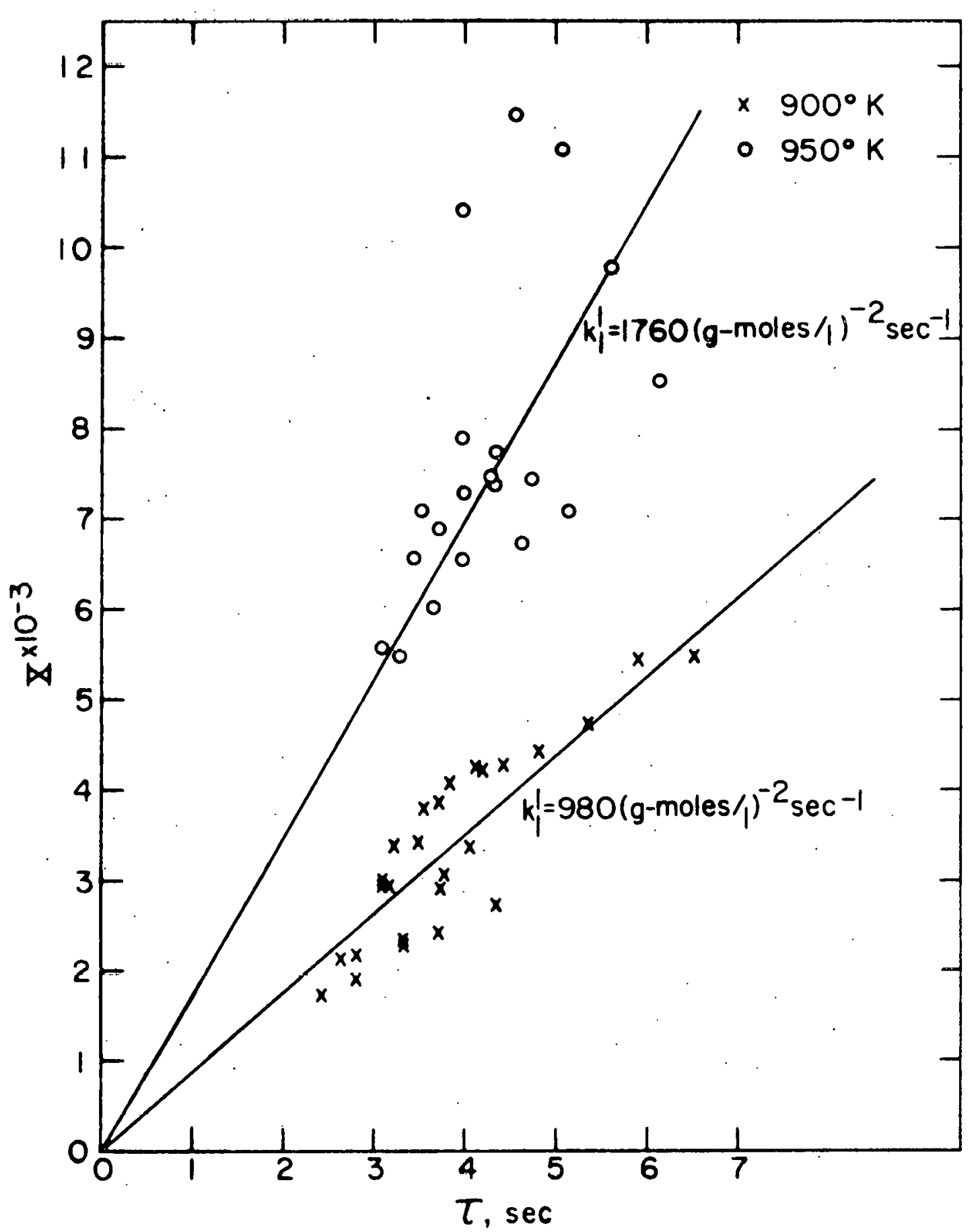

Figure 10. $\mathrm{X}$ as a function of space-time for model $\mathrm{E}$ (five-pass reactor) 
The straight lines yield the constants $k_{1}^{\prime}=980(g-m o l e s / 2)^{-2} \sec ^{-1}$ at $900^{\circ} \mathrm{K}, \mathrm{k}_{1}^{\prime}=1760(\mathrm{~g} \text {-moles } / \ell)^{-2} \mathrm{sec}^{-1}$ at $950^{\circ} \mathrm{K}, \mathrm{A}=6.6 \times 10^{7}(\mathrm{~g} \text {-moles } / \ell)^{-2}$ $\sec ^{-1}$, and $\mathrm{E}=83.24 \mathrm{KJ} / \mathrm{g}$-mole.

Because the experimental data seem to fit only the two forward reaction rate expressions, models $B$ and $E$, these two models were used to derive the combined forward and reverse reaction rate expressions (see Theory Section, p. 24). Table 4 lists the values of X calculated from Equations (54), (60), and (66) at 900 and $950^{\circ} \mathrm{K}$. The assumption that the reverse reaction can be neglected can be easily justified by. comparison of those values.

Mode1 B can be justified mechanistically in that it implies that the reaction which forms chlorine radicals, $\mathrm{Cl}_{2} \rightleftharpoons 2 \mathrm{Cl}$, is rate controling. The slightly greater dispersion of data at $950^{\circ} \mathrm{K}$ might suggest that the formation of chlorine radicals is not the only ratecontrolling step at higher temperatures.

The dissociation of chlorine gas into chlorine radicals was found to occur in a few photochemical reactions $(5,13,30)$. The Gibbs free energy for this reaction is $33.77 \mathrm{Kcals} / \mathrm{g}-\mathrm{mole}$ at $900^{\circ} \mathrm{K}$ and 32.37 $\mathrm{Kcals} / \mathrm{g}$-mole at $950^{\circ} \mathrm{K}$. And the equilibrium constant, $\mathrm{K}=\frac{[\mathrm{Cl} \cdot]^{2}}{\left[\mathrm{Cl}_{2}\right]}$, is $6.3 \times 10^{-9}$ at $900^{\circ} \mathrm{K}$ and $3.6 \times 10^{-8}$ at $950^{\circ} \mathrm{K}(25)$.

If the activated-complex theory is applied, the elementary reaction for model $\mathrm{B}$ and model $\mathrm{E}$ can be expressed as $\mathrm{Cl}_{2} \longrightarrow\left(\mathrm{Cl}_{2}^{\neq}\right)$ $\longrightarrow$ products (Reaction III) and $2 \mathrm{Cl}_{2}+\mathrm{H}_{2} \mathrm{O} \longrightarrow\left(\mathrm{Cl}_{2} \cdot \mathrm{H}_{2} \mathrm{O} \cdot \mathrm{Cl}_{2}^{\neq}\right.$) $\longrightarrow$ products (Reaction IV). For Reactions III and IV, at 925\% , the Gibbs free energy of activation are 106.58 and $42.92 \mathrm{Kcals} / \mathrm{g}-\mathrm{mole}$, the enthalpy of activation are 14.00 and $14.38 \mathrm{Kcals} / \mathrm{g}-\mathrm{mole}$, and the 
entropy of activation are $-51.90 \mathrm{cals} / \mathrm{g}-\mathrm{mole} \cdot \mathrm{O}_{\mathrm{K}}$ and $-30.85 \mathrm{cals} /$ g-mole. ${ }^{\circ}$. Detalled calculations are shown below.

Ea:= Arrhenius activation energy

$\Delta G^{\ddagger}=$ Gibbs free energy of activation

$\Delta \mathrm{H}^{\ddagger}=$ enthalpy of activation

$\Delta S^{\neq}=$entropy of activation

$\mathrm{n}=$ order of the reaction

$k=$ Boltzmann constant $=1.30 \times 10^{-16}$ erg. $/^{\circ} \mathrm{K}$

$\mathrm{h}=\mathrm{P}$ lanck constant $=6.624 \times 10^{-27} \mathrm{erg} \cdot \cdot \mathrm{sec}$.

$\mathrm{T}=$ absolute temperature

For model B:

$$
\begin{aligned}
& k_{1}=\frac{k T}{h} \cdot \exp \cdot\left(-\Delta C^{\ddagger} / R T\right) \\
& =\frac{k T}{h} \cdot \exp \cdot\left(\Delta S^{\ddagger} / R\right) \cdot \exp \cdot\left(-\Delta H^{\ddagger} / R T\right) \\
& \Delta \mathrm{H}^{\ddagger}=\mathrm{Ea}-\mathrm{nRT}=15.84-1.987 \times 925 \times 10^{-3}=14.00 \mathrm{Kcal} / \mathrm{g}-\mathrm{mo} \cdot \mathrm{le} \\
& =58.57 \mathrm{~K} . \mathrm{J} \cdot / \mathrm{g}-\mathrm{mole} \\
& k_{1}=A \cdot \exp \cdot(-E a / R T) \\
& =224 \cdot \exp \cdot\left(-15.84 \times 10^{3} / 1.987 \times 925\right) \\
& =0.0405\left(\sec ^{-1}\right) \\
& 0.0405=\frac{1.3 \times 10^{-16} \cdot 925}{6.624 \times 10^{-27}} \cdot \exp \cdot\left(\Delta S^{\neq} / R\right) \cdot \exp \cdot\left(-1.4 .00 \times 10^{3} /\right. \\
& \text { i.987 - 925) } \\
& \Delta \mathrm{s}^{\phi}=\Delta \mathrm{H}^{\neq}-\mathrm{L} \Delta \mathrm{s}^{\neq} \equiv 58.57-925 \times(-0.0519) \\
& =106.58 \mathrm{Kcals} / \mathrm{mole}=446.14 \mathrm{~K} . \mathrm{J} . / \mathrm{g}-\mathrm{mole}
\end{aligned}
$$


For model E:

$$
\begin{aligned}
& k_{1}^{\prime}=\frac{k T}{h} \cdot \exp \cdot\left(\Delta S^{\neq} / R\right) \cdot \exp \cdot\left(-\Delta H^{\ddagger} / R T\right) \\
& \Delta \mathrm{H}^{\ddagger}=\mathrm{Ea}-\mathrm{nRT}=19.89-3 \cdot 1.987 \cdot 925 \times 10^{-3} \\
& =14.38 \mathrm{Kca} 1 \mathrm{~s} / \mathrm{g}-\mathrm{mole}=60.18 \mathrm{~K} . \mathrm{J} . / \mathrm{g}-\mathrm{mole} \\
& k_{1}^{\prime}=6.6 \times 10^{7} \cdot \exp \cdot\left(-19.89 \times 10^{3} / 1.987 \cdot 925\right) \\
& =.1314 .68(\mathrm{~g}-\mathrm{moles} / \ell)^{-2} \cdot \mathrm{sec}^{-1} \\
& 1314.68=\frac{1.30 \times 10^{-16} \cdot 925}{6.624 \times 10^{-27}} \cdot \exp \cdot\left(\Delta S^{\ddagger} / R\right) \cdot \exp \cdot\left(-14.38 \times 10^{3} /\right. \\
& 1.987 \cdot 925) \\
& \Delta \mathrm{S}^{\neq}=-30.85 \mathrm{cals} / \mathrm{g}-\mathrm{mole} \cdot \mathrm{o}_{\mathrm{K}}=-129.07 \mathrm{joules} / \mathrm{g}-\mathrm{mole} \cdot \mathrm{o}_{\mathrm{K}} \\
& \Delta G^{\ddagger}=14.38-925 \cdot 0.03085=42.92(\mathrm{Kcals} / \mathrm{g}-\mathrm{mole}) \text {. } \\
& =179.65 \text { (K.J./g-mole) }
\end{aligned}
$$

Comparing these thermodynamic properties, the entropy of activation for model E seems to be more realistic than that for mode1 B. However, mode1 $\mathrm{B}$, shown in Figure 5, appears to give a slightly better fit of the data than model $\mathrm{E}$, shown in. Figure 10 .

The results achieved by using the current equipment cannot really prove that the radicals exist in the reverse Deacon reaction at 900 and $950^{\circ} \mathrm{K}$. There are a few methods that can be used to help determine whether the reaction system contains radicals (30). Even if the 
presence of radicals could be confirmed, there would exist many possible mechanisms for the reverse Deacon reaction. Just like the controversial mechanisms of many photochemical and thermal reactions, unless solid proof can be found, the reaction mechanism will remain undetermined. But, by using better designed equipment and by applying the statistical sensitivity analysis method, an experimental reaction rate expression can still be justified $(3,42)$.

The space-time required in those experiments suggests that a catalyst would be justified to increase the reaction rate in a commercial scale reactor operating at 900 and $950^{\circ} \mathrm{K}$. The catalyst candidates, which must not melt and sublimate at those specific temperatures, are extremely limited, however. Cooling of the product gaseous stream would probably be rapid enough to avoid a significant amount of the reverse reaction. But the energy loss of this highly irreversible cooling process should also be considered as a disadvantage of this system. If the reaction temperature is raised to $1100 \% \mathrm{~K}$, for which the equilibrium conversion is $66.5 \%$ for equal-molal feed, catalysts may not be necessary. But the cooling process for avoiding the reverse reaction would be an engineering problem, because the temperature of the product gaseous stream needs to be lowered in a very short time. KI solution was used to trap the unreacted chlorine most of the time. $\mathrm{NaOH}$ and $\mathrm{Na}_{2} \mathrm{~S}_{2} \mathrm{O}_{3}$ solutions were also tried as substitutes for KI solution. But the results were not satisfactory because of the following reasons: 


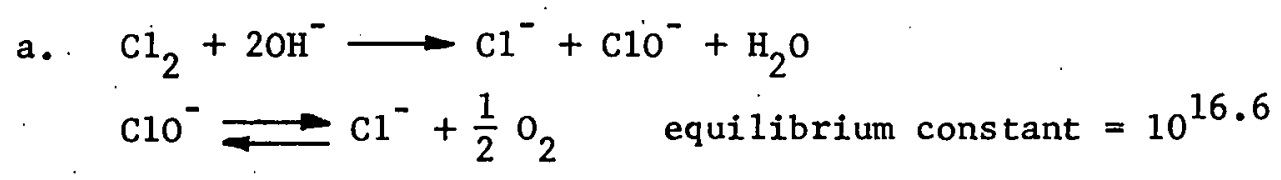

The kinetics for the second reaction are not available. But the thermodynamic data shows the equilibrium really favors the right hand side. The resulting oxygen evolved might have caused the unsatisfactory results.

b. $\mathrm{H}_{2} \mathrm{~S}$ was generated when $\mathrm{Na}_{2} \mathrm{~S}_{2} \mathrm{O}_{3}$ solution was used (56). The oxygen evolution rates were therefore not accurate because of the presence of $\mathrm{H}_{2} \mathrm{~S}$.

The Results of One-Pass Reactor at $900{ }^{\circ} \mathrm{K}$ and the Discussion of Deviations from Plug Fiow Assumptions

The results are given in Table 5. Comparing the values of $X$ calculated from Equations (43), (54), and (60) shows that the reverse reaction can be neglected for model $\mathrm{B}$. The same conclusion can be made for model $\mathrm{E}$ by comparing the values of $\mathrm{X}$ calculated from Equations (46) and (60). Plots of Equations (44) and (47) are shown in Figures 11 and 12 respectively. Those nine data points in Figure 11 apparent1y can be put into three groups. Each group forms a straight line which passes through the origin. Figure 12, although not that obviously, also shows the same pattern. It is noticed that the group with the largest slope happens to be the group with the highest flow rates in both cases. 
Table 5. Experimental results of one-pass reactor at $900 \mathrm{~K}^{\mathrm{a}}$

\begin{tabular}{|c|c|c|c|c|c|c|c|c|c|}
\hline $\begin{array}{c}\text { Run } \\
\text { number }\end{array}$ & $\begin{array}{l}\mathrm{U}_{\mathrm{Cl}_{2}}^{\mathrm{b}}{ }^{\mathrm{b}} \\
\text { (cc/min.) }\end{array}$ & $\begin{array}{c}\mathrm{U}_{\mathrm{H}_{2} \mathrm{O}}^{\mathrm{b}} \\
\text { (cc/min.) }\end{array}$ & $\begin{array}{c}\mathrm{U}^{\mathrm{o}^{\mathrm{b}}} \\
(\mathrm{cc} / \mathrm{min} .)\end{array}$ & $\begin{array}{c}\top \\
\left(\mathrm{sec}^{2}\right)\end{array}$ & $\begin{array}{l}\mathrm{C}_{\mathrm{C} 1_{2}}^{\mathrm{o}} \times 10^{3} \\
(\mathrm{~g} \text {-moles } / \ell)\end{array}$ & $\begin{array}{l}\mathrm{C}_{\mathrm{H}_{2} \mathrm{O}}^{\mathrm{o}} \times 10^{3} \\
(\mathrm{~g} \text {-moles/l) }\end{array}$ & $\begin{array}{c}\mathrm{U}_{\mathrm{O}_{2}}{ }^{\mathrm{c}} \\
\text { (cc/min.) }\end{array}$ & $\mathrm{N}_{1}$ & $\begin{array}{l}\mathrm{x}_{\mathrm{C} 1_{2}}^{\mathrm{f}} \\
(\%)\end{array}$ \\
\hline $\mathrm{C} 1$ & 142 & 358 & 500 & 20.64 & 3.848 & 9.702 & 5.91 & 2.521 & 25.40 \\
\hline $\mathrm{C} 2$ & 99 & 358 & 457 & 28.84 & 2.935 & 10.615 & 5.07 & 3.616 & 31.25 \\
\hline C3 & 164 & 358 & 522 & 19.78 & 4.257 & 9.293 & 6.46 & 2.183 & 24.03 \\
\hline C4 & 164 & 39 & 203 & 50.92 & 10.947 & 2.603 & 3.47 & 0.238 & 12.91 \\
\hline C5 & 99 & 39 & 138 & 74.92 & 9.721 & 3.829 & 2.93 & 0.394 & 18.06 \\
\hline C6 & 142 & 39 & 181 & 57.04 & 10.630 & 29.20 & 3.23 & 0.275 & 13.88 \\
\hline C7 & 142 & 187 & 329 & 31.36 & 5.848 & 7.702 & 4.20 & 1.317 & 18.05 \\
\hline C8 & 99 & 187 & 286 & 36.10 & 4.690 & 8.860 & 3.71 & 1.889 & 22.86 \\
\hline C9 & 164 & 187 & 351 & 29.42 & 6.331 & 7.219 & 4.75 & 1.140 & 17.67 \\
\hline
\end{tabular}

${ }^{a}$ Detailed calculations are shown in Appendix II.

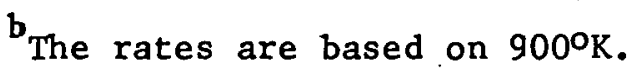

${ }^{c}$ The rates are based on $295^{\circ} \mathrm{K}$. 
Table 5. Continued

\begin{tabular}{lccccc}
\hline $\begin{array}{c}\text { Run } \\
\text { number }\end{array}$ & Equation (43) & Equation (54) & Equation (60) & Equation (46) & Equation (66) \\
\hline C1 & 0.2986 & 0.3004 & 0.2986 & 10262 & 10155 \\
C2 & 0.3814 & 0.3839 & 0.3815 & 16257 & 16099 \\
C3 & 0.2803 & 0.2820 & 0.2803 & 9066 & 8791 \\
C4 & 0.1419 & 0.1460 & 0.1422 & 8367 & 8302 \\
C5 & 0.2059 & 0.2134 & 0.2064 & 8047 & 8996 \\
C6 & 0.1536 & 0.1580 & 0.1538 & 5085 & 8 \\
C7 & 0.2031 & 0.2042 & 0.2031 & 8161 & 5569 \\
C8 & 0.2649 & 0.2667 & 0.2649 & 5489 & 875 \\
C9 & 0.1986 & 0.1999 & 0.1986 & 5 & 5 \\
\hline
\end{tabular}




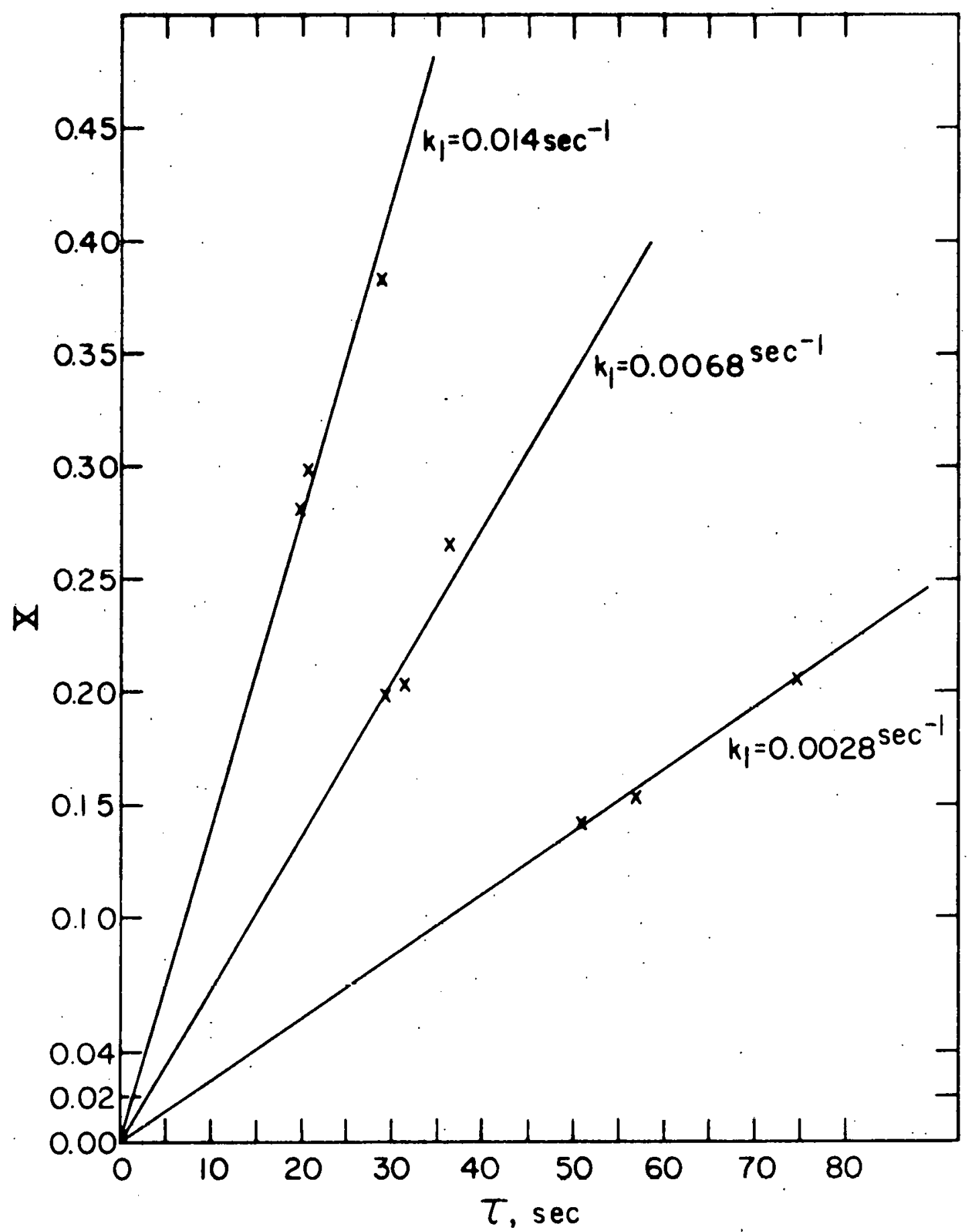

Figure 11. $\mathrm{X}$ as a function of space-time for model $\mathrm{B}$ at $900^{\circ} \mathrm{K}$ (one-pass reactor) 


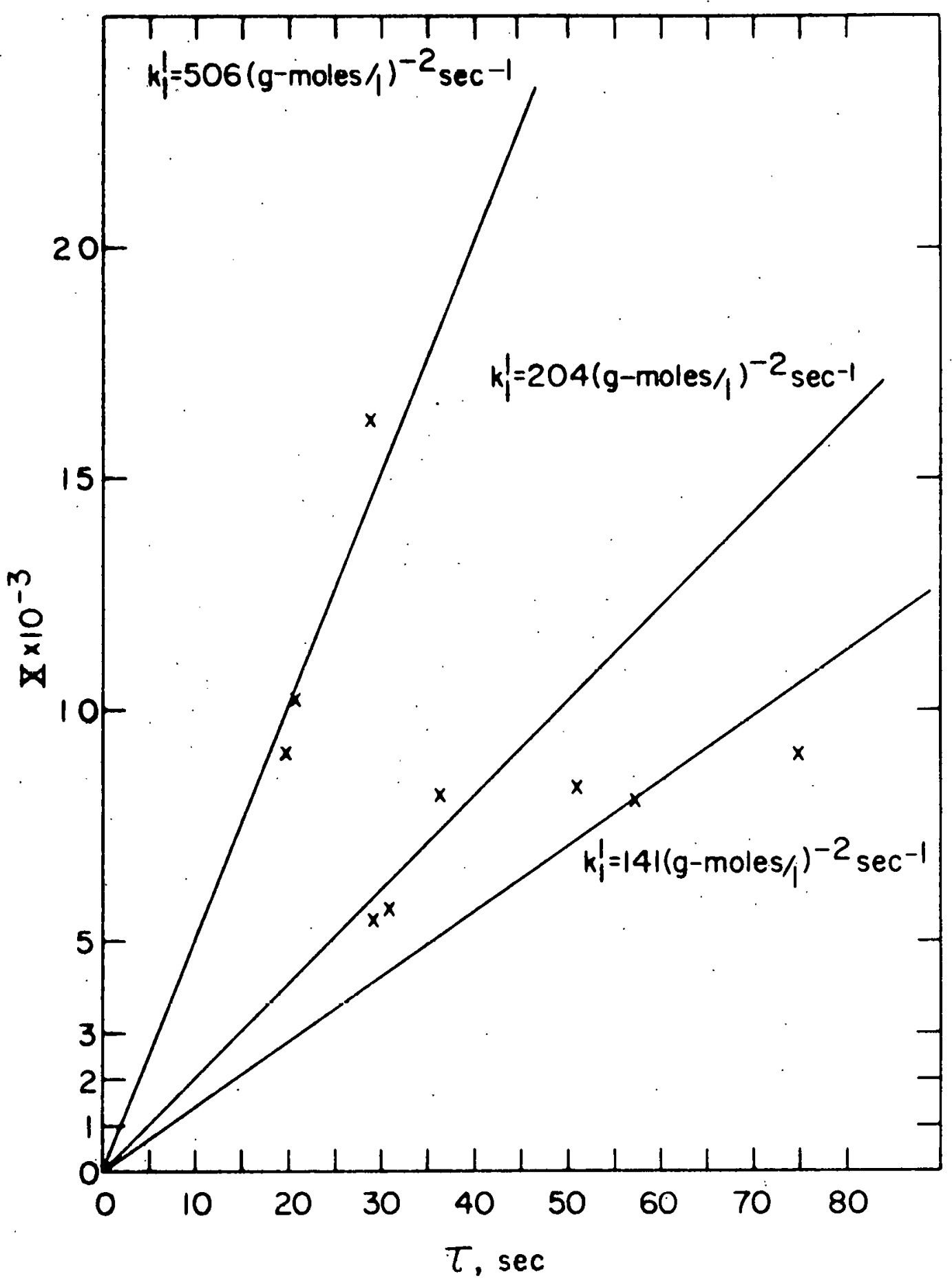

Figure 12. $X$ as a function of space-time for model $\mathrm{E}$ at $900 \mathrm{~K}^{\mathrm{K}}$ (one-pass Leaclul) 
Table 6 lists the highest and the lowest Reynolds numbers at the reactor entrance. It shows that the flow patterns of all the experiments are laminar.

Table $6 .^{a}$

Run number
Reynolds No.

Highest Lowest

49.67

37.35

6.30
19.34

19.13

2.49

${ }^{a}$ Detailed calculations are shown in Appendix VI.

According to Denbigh (17), transverse temperature gradients, transverse and longitudinal diffusion, and transverse velocity gradient cause deviations from plug flow.

The reverse Deacon reaction is endothermic at $900^{\circ} \mathrm{K}$. The heat of reaction when one g-mole of steam reacts completely with one g-mole of chlorine is $14.223 \mathrm{Kcals}$. The conversion and the molal flow rates of both reactants are so low for those experiments that an isothermal reactor can be assumed without much error. Therefore, the transverse temperature gradient is not the cause for deviation from $p$ lug flow in this case.

Because the flow patterns are laminar, the diffusional mechanism which is operative is the ordinary molecular diffusion. The effect of molecular diffusion for gases reduces the influence of the velocity 
distribution in causing a deviation from plug flow. However, this positive effect is offset by the effect of the longitudinal molecular diffusion which is determined mainly by the ratio of the length of reactor to its diameter. That ratio of the five-pass reactor is 210 , which is fourteen times larger than the value of the one-pass reactor. That might be one of the reasons for the better performance of the five-pass reactor.

The existence of velocity gradients at right-angles to the direction of fluid flow is the third form of deviation from the plug flow. The fluid velocity is smaller in the vicinity of the wall than it is near the reactor center. Therefore, elements of fluid moving closer to the wall have a larger space-time and tend to react to a greater extent. Because the velocity profile of turbulent flow is much flatter than the velocity profile of laminar flow, it is obvious that the greatest deviation from the reactor performance estimated by using the plug flow assumptions occurs in the case of laminar flow. In actual practice the velocity gradient is usually a great deal less significant than the influences of transverse temperature gradients and transverse diffusion which have been discussed previously. Therefore, even though the flow patterns of those experiments are laminar, the deviation caused by the velocity gradients is considered negligible.

Besides the three causes mentioned above, the effect of imperfect mixing is considered to be very serious in causing the deviation. With higher Reynolds numbers the mixing of the reactants should be better. Refer back to Figure 11, the group with the highest value of 
$k_{1}$ is the group with the largest flow rates and this $k_{1}$ is less than half of the $k_{1}$ from five-pass reactor. The average Reynolds number for those experiments using the five-pass reactor is seven times larger than for the one-pass reactor.

Estimation of the Reactor Size

Conditions :

1. A $2000 \mathrm{MW}$ reactor is used to drive a thermochemical water splitting reaction which is $50 \%$ efficient. Therefore, the available chemical energy in the hydrogen is $1000 \mathrm{MW}$.

2. The reverse Deacon reaction (Reaction I) is the oxygengeneration step. And Equation (50) is its reaction rate expression.

The heat of dissociation of one g-mole of liquid water at $298.15^{\circ} \mathrm{K}$ is $68.32 \mathrm{Kcals}$. The theoretical molal production rate of hydrogen is $1.26 \times 10^{7} \mathrm{~g}$-moles/hr $\left(\frac{2 \times 10^{6} \mathrm{KW} \times 50 \% \times 8.6 \times 10^{2} \mathrm{Kcals} / \mathrm{hr}}{68.32 \mathrm{Kcals} / \mathrm{g}-\mathrm{mole}}\right)$. The theoretical molal production rate of oxygen, which is half of the rate of hydrogen, is $6.3 \times 10^{6}$ g-moles/hr. For Reaction I with $\mathrm{N}_{1}=1$ at $900^{\circ} \mathrm{K}$ and $50 \mathrm{atms}$, the equilibrium constant, $\mathrm{K}_{\mathrm{eq}}$,' is 0.1604 and the

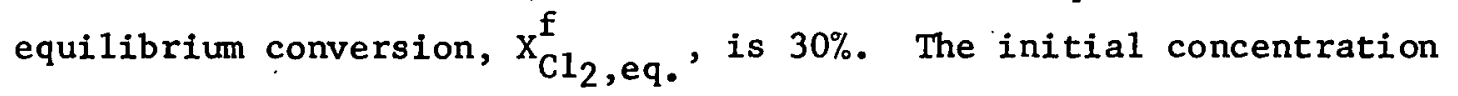
of chlorine, $\mathrm{C}_{\mathrm{Cl}_{2}}^{\circ}$, is $0.339 \mathrm{~g}$-moles/l (by applying the ideal gas law).

Equation (53) becomes

$$
\tau=7.81 \int_{0}^{\mathrm{X}_{\mathrm{Cl}}^{\mathrm{f}}}\left(4+\mathrm{X}_{\mathrm{Cl}}\right)\left\{1-\mathrm{X}_{\mathrm{Cl}}{ }_{2}-\frac{20.53 \mathrm{x}_{\mathrm{Cl}}^{5 / 2}}{\left(4+\mathrm{x}_{\mathrm{Ci}}\right)^{1 / 2}\left(1-\mathrm{x}_{\mathrm{Cl}}\right)}\right\}^{-1} d \mathrm{x}_{\mathrm{Cl}{ }_{2}}
$$


If the final conversion based on chlorine is $30 \%$, which is $100 \%$ of the equilibrium conversion, the space-time in Equation (79) will be 135.33 seconds and the total flow rate entering the reactor will be $8.4 \times 10^{7} \mathrm{~g}$-moles/hr $\left(1.24 \times 10^{8} \mathrm{l} / \mathrm{hr}\right)$. Therefore the reactor volume, which is the product of the space-time and the total flow rate, is $4660 \mathrm{~m}^{3}$.

If the final conversion is $24 \%$, which is $80 \%$ of the equilibrium conversion, the space-time will be 9.57 seconds and the flow rate will be $1.05 \times 10^{8} \mathrm{~g}$-moles/l $\left(1.55 \times 10^{8} \mathrm{l} / \mathrm{hr}\right)$. Then the reactor volume is $412 \mathrm{~m}^{3}$ which is only one-eleventh of the volume for $100 \%$ of equilibrium.

If the value of $\mathrm{N}_{1}$ is increased from 1 to 20 , the equilibrium conversion is increased from $30 \%$ to $88.1 \%$. Equation (53) becomes

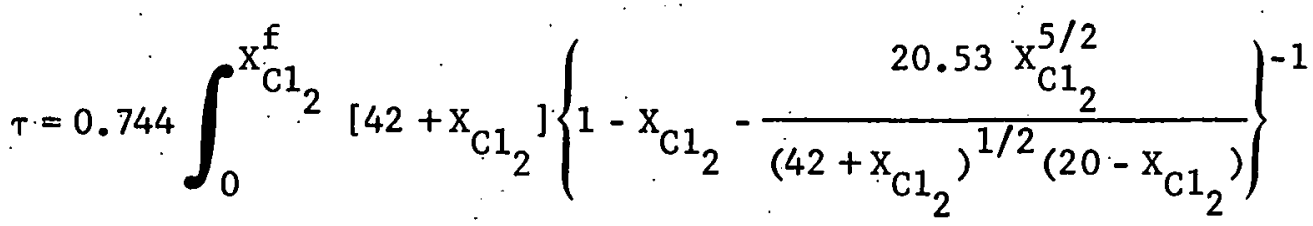

$$
\begin{aligned}
& \mathrm{dx}_{\mathrm{Cl}_{2}}
\end{aligned}
$$

If the conversion is $70 \%$ which is $80 \%$ of the equilibrium conversion, the space-time in Equation.(80) will be 38.31 seconds and the total feed rate will be $3.78 \times 10^{8}$ g-moles/hr $\left(5.58 \times 10^{8} \mathrm{l} / \mathrm{hr}\right)$. The reactor volume is $5940 \mathrm{~m}^{3}$ which is fourteen times the volume for equal-molal feed with $24 \%$ conversion.

If the conversion is $80 \%$ which is almost $91 \%$ of the equilibrium conversion, the space-time will be 56.33 seconds and the total flow rate will be $3.31 \times 10^{8} \mathrm{~g}$-moles/hr $\left(4.88 \times 10^{8} \mathrm{l} / \mathrm{hr}\right)$. Therefore the 
reactor volume is $7640 . \mathrm{m}^{3}$ which is eighteen times of the volume for equal-molal feed with $24 \%$ conversion.

The increase of the value of $\mathrm{N}_{1}$ will yield higher equilibrium conversion but also higher total flow rate and longer space-time. Therefore a larger size of reactor will be needed.

If the reactor temperature is raised to $950^{\circ} \mathrm{K}$, the equilibrium constant, $\mathrm{K}_{\mathrm{eq}}$, is 0.2365 and the equilibrium conversion, $\mathrm{x}_{\mathrm{Cl}}^{\mathrm{f}}$, is $34 \%$. With $\mathrm{N}_{1}=1$ Equation (53) becomes

$$
\tau=4.9 \int_{0}^{\mathrm{X}_{\mathrm{Cl}}^{\mathrm{f}}}\left(4+\mathrm{x}_{\mathrm{C} 1_{2}}\right)\left\{1-\mathrm{x}_{\mathrm{Cl} 1_{2}}-\frac{13.55 \mathrm{x}_{\mathrm{C1}}^{5 / 2}}{\left(4+\mathrm{x}_{\mathrm{Cl}{ }_{2}}\right)^{1 / 2}\left(1-\mathrm{x}_{\mathrm{Cl}}\right)}\right\}^{-1} \mathrm{dx} \mathrm{C}_{2}
$$

If. the final conversion is $24 \%$ which is $70 \%$ of the equilibrium conversion, the space-time in Equation (81) will be 5.61 seconds and the total feed rate will be $1.05 \times 10^{8} \mathrm{~g}$-moles/hr $\left(1.64 \times 10^{8} \mathrm{l} / \mathrm{hr}\right)$. The reactor volume is $256 \mathrm{~m}^{3}$ which is 0.62 times of the volume for the case of $900^{\circ} \mathrm{K}, 50 \mathrm{~atm}$, and $24 \%$ conversion.

The increase of the reaction temperature yields a more reasonable reactor volume. The avallable dala is nut accurate enough to show what the reactor size will be if the reaction temperature is raised to $1000^{\circ} \mathrm{K}$ or even $1100^{\circ} \mathrm{K}$. But one thing can be sure, further reduction of the reactor size will be necessary to make this process successful. And that can be achieved either by raising the reactor temperature or by applying a suitable catalyst to the reaction. 


\section{RECOMMENDATION}

There were no parameters other than the oxygen evolution rate to. double-check the values of the reaction conversion for these experiments. A total material balance around the system was. not obtained. In order to achieve more precise results, two methods which can be used to detect the concentration of each species in the gaseous product stream are suggested. One method is to use a gas chromatograph to analyze the samples taken from the exit of the reactor. The samples have to be quenched to stop both forward and reverse reactions. If the temperature is quenched below $100^{\circ} \mathrm{C}$, the steam will condense and absorb some hydrogen chloride and chlorine. Then an accurate analytic method, which wili be able to detect the precise amount of water, hydrogen chloride, and chlorine present in the liquid phase, has to be developed. If the reaction is quenched above $100^{\circ} \mathrm{C}$ so that the steam will not condense, the samples can be directly injected into the gas chromatograph which is also run at about $100^{\circ} \mathrm{C}$. The other method is to use a spectrophotometer at the exit of the reactor to photometrically measure the concentration of certain species at a certain wave length $(45,46)$. The unchanged concentration reading will mean the system has already reached the steady state. Then a new wave length is set to get the concentration of another species.

The chlorine rotameter was calibrated at 5 psi by the manufacturer. The current chlorine regulator delivery pressure range of 4-100 psi is not precise enough to control the pressure at 5 psi. The inaccuracy of the pressure will affect the chlorine flow rates, and therefore 
the values of the space-time, the conversion based on the chlorine flow rate, and the molal feed ratio. This problem can be solved by installing a line regulator with delivery pressure range of $0.5-8$ psi or changing the original regulator to a corrosion-resistance regulator with delivery pressure range of 4-35 psi.

Thorough mixing is among the plug flow assumptions made for this system. Therefore, a nozzle type of mixing zone, which will increase the velocity of the gaseous mixture drastically, will be an ideal device to ensure perfect mixing.

Catalyst might be necessary for the reverse Deacon reaction to be economically feasible. A vertical reactor will be better to use as a catalytic system than a horizontal one. 


\section{BI BLIOGRAPHY}

1. Abraham, B. M., and F. Schreiner. 1974. General principles underlying chemical cycles which thermaliy decompose water into elements. Ind. Eng. Chem., Fundam. 13(4): 305-310.

2. Arnold, C. W., and K. A. Kobe. 1952. Thermodynamics of the Deacon process. Chem. Eng. Prog. 48(6): 293-296.

3. Atherton, R. W., R. B. Schainker, and E. R. Ducot. 1975. On the statistical sensitivity analysis of models for chemical kinetics. A.I.Ch.E. Journal 21(3): 441-448.

4. Barstow, E. O., and S. B. Heath. 1932. U.S. Patent 1,874,225 (to The Dow Chemical Company). Aug. 30 .

5. Bodenstein, M. 1931. Some remarks about "Chlorknallgas". Trans. Faraday Soc. 27: 413-430.

6. Bowmann, M. G. 1974. Fundamental aspects of systems for the thermochemical production of hydrogen from water. IA-UR-74-1459. (Los Alamos Scientific Lab., Los Alamos, NM.)

7. Brokris, J. 0. 1972. The electrochemistry of cleaner environments. Plenum Press, New York, NY.

8. Burger, J. M., P. A. Lewis, R. J. Isler, F. J. Salizano, and J. M. King. 1974. Energy storage for utilities via hydrogen systems. BNL-19266. Paper presented at 9th Intersociety Energy Conversion Engineering Conference, San Francisco, California, Aug. 26-30, 1974.

9. Chao, R. E. 1975. Thermochamical hydrogen production. An assessment of nonideal cycles. Ind. Eng. Chem., Process Des. Dev. $14(3): 276-279$.

10. Chao, R. E. 1974. Thermochemical water decomposition process. Ind. Eng. Chem., Prod. Res. Develop. 13(2): 94-101.

11. Chemical Abstracts. 1916. Hydrochloric acid. Soc. Italiana Elettrochimica. Swed., 40: 354, March 15, 1916. Chemical Abstracts 10: 1581 .

12. Chemical and Englneering News. 1973. Hydrogen sought via thermochemical methods. Chemical and Engineering News 51 (Sept. 3): 32 .

13. Chemical Society. 1931. Discussion on the critical increment of livmugemeus redeitun. The themical society, Burlington House, London, England. 
14. Chizhik, A. A. 1932. The preparation of hydrochloric acid from chlorine and steam in the presence of carbon. J. Chem. Ind. (Moscow) No. 9: 37-39.

15. Chopey, N. P. 1972. Hydrogen: Tomorrow's fuel. Chem. Eng. 79 (Dec. 25): 24-26.

16. Deacon reaction (Patents):

Balcar, F. R. 1942. U.S: Patent 2,271,056 (to Air Reduction Company). Jan. 27.

Davis, C. W., F. A. E. Antioch, and R. G. Ellis. 1951. U.S: Patent 2,547,928 (to The Dow Chemical Company). Apr. 10.

Diamond Alkali Company. 1952. Brit. Patent 676,667. July 30 .

Institut Francais du Pétrole des Carburants et Lubrifiants. 1960. Deutsches Patentamt 1,0760635. Mar, 3.

Johnson, A. J., and A. J. Cherniavsky. 1953. U.S. Patent $2,644,846$ (to She11 Development Company). July 7.

Johnson, A. J., and A. J. Cherniavsky. 1956. U.S. Patent $2,746,844$ (to She 11 Development Company). May 22.

Krekeler, H., and H. Schlecht. 1952. Deutsches Patentamt 857,796 (to Badische Anilin-\& Soda-Fabrik). Dec. 1.

VEB Farbenfabrik Wolfen. 1960. Deutsches Patentamt 1,086,677. Aug. 11.

VEB Farbenfabrik Wo1fen. 1960. Deutsches Patentamt 1,078,100. Mar. 24.

VEB Farbenfabrik Wolfen. 1960. Deutsches Patentamt 1,088,933. Sept. 15.

17. Denbigh, K. 1965. Chemical reactor theory. Cambridge University Press, London, England.

18. Euratom. 1973. Hydrogen production from water using nuclear heat. Progress Report No. 3: EUR/C-IS/35/73e.

19. Euratom. 1973. Musso, G., and J. Flatt. Reaction of water with chlorine. Pruyress Report No. 3: EUR-5059e.

20. Falckenstein, K. V. 1907. Equilibrium of the Deacon process. 7. Phyolk. Chem, 50: 313-335. 
21. Fein, E., and F. J. Salzano. 1974. The hydrogen economy - a utility perspective. C74 099-8. A paper recommended by the IEEE Power Generation Committee of the IEEE Power Engineering Society for presentation at the IEEE PES winter meeting, New York, N.Y., Jan. 27-Feb. 1.

22. Funk, J. E. 1974. The generation of hydrogen by the thermal decomposition of water. Proc. 9th IECEC, San Francisco, Calif., Aug. 26-30.

23. Funk, J. E., and R. M: Reinstrom. 1965. Energy requirements in the production of hydrogen from water: Allison Division - General Motor Co. Contract DA-44-009-AMC-855(X) - U.S. Army.

24. Funk, J. E., and R. M. Reinstrom. 1966. Energy requirements in the production of hydrogen from water. Ind. Eng. Chem. Proc. Des. Deve1. 5(3): 336-342.

25. Giaugue, W. F., and R. Overstreet. 1932. The hydrogen, ch1orine, hydrogen chloride equilibrium at high temperature. J. Am. Chem. Soc. 54: 1731-1744.

26. Gibbs, H. D. 1920. The production of hydrochloric acid from chlorine and water. J. Ind. Eng. Chem. 12: 538-541.

27. Gregory, D. P. 1973. Hydrogen economy. Sci. Amer. 228(1): 13-21.

28. Gregory, D. P. 1972. Hydrogen: Transportable storable energy medium. Amer. Chem. Soc., Div. Fuel. Chem., Prep. 16(4): 88-94.

29. Gregory, D. P., D. Y. C. Ng, and G. M. Long. 1972. The electrochemistry of cleaner environments. Plenum Press, New York, N.Y.

30. Hinshelwood, C. N. 1940. Kinetics of chemical change. Oxford University Press, England.

31. Hirschkind, W. 1925. Manufacture of hydrochloric acid from chlorine. Ind. Eng. Chem. 17: 1071-10.73.

32. Hirschkind, W., and C. W. Schedler, 1928. U.S. Patent 1,695,552, Dec. 18.

33. Johnstone, H. F. 1948. Chlorine production - nonelectrolytic processes. Chem. Eng. Prog. 44(9): 657-668.

34. Jones, A. 1965. Kinetics of the oxidation of hydrogen chloride. Ph.D. Dissertation. Yale University. 
35. Knoche, K. F., H. Cremer, and G. Steinborn. 1975. A thermochemical process for hydrogen production. Presented at the Hydrogen Energy Conference, Miami, Florida, March 3-5.

36. Lessing, L. 1972. The coming hydrogen economy. Fortune $86(5)$ : 138.

37. Levenspie1, 0. 1972. Chemical reaction engineering. Second edition. John Wiley \& Sons, Inc., New York.

38. Martin, G. 1915. Chlorine and chlorine products. D. Appleton and Company, New York.

39. Maugh, T. H. 1972. Hydrogen: Synthetic fuel of the future. Science 178(4063): 849-852.

40. Ohkawa, T. 1949. Japan Patent 180,186 . Sept. 8.

41. Padovani, C., E. De Dartholomaeis, and C. Siniramed. 1939. Synthesis of hydrochloric acid by reaction of methane, water vapor, and chlorine. Atti $\mathrm{X}^{\circ}$ congr. Intern. Chim. 4: 51-56.

42. Pritchard, D. J., and D. W. Bacon. 1975. Statistical assessment of chemical kinetics models. Chem. Eng. Sci. 30: 567-574.

43. Reaction of $\mathrm{H}_{2} \mathrm{O}$ and $\mathrm{Cl}_{2}$ in the presence of a reducing agent (Patents):

Behrman, A. S. 1932. U.S. Patent 1,843,196. Feb. 2 .

Doma, G., and G. Andreani. 1922. Brit. Patent 189,723.' May 19.

Kenkyûjo, R. 1935. Japan Patent 111,410. July 2.

Krebs et Cie. Soc. anon. 1951. Fr. Patent $992,928$. Oct. 24.

Paulus, H. W. 1922. U.S. Patent 1,420,209 (to Roya1 Baking

Powder Company). June 20.

Peter, A. H. 1917. U.S. Patent 1,229,509 (to Royal Baking Company). June 12 .

Randaccio, C. 1954. Ital. Patent 507,052. Dec, 28.

Rosenstein, L. 1924, U.S. Patent 1,485,816 (to Great Westem Electro Chemical Company). Mar. 4.

44. Reed, R. M. 1966. Kirk-Othmer Encyclopedia of Chemical Technology. Vol. 11. 2nd ed. Interscience, New York. 
45. Rosser, W. A., Jr. 1959. The kinetics of oxidation of $\mathrm{HBr}$. J. Phys. Chem. 63: 1753-1755.

46. Rosser, W. A., Jr., and H. Wise. 1960. Kinetics of the gas phase oxidation of hydrogen chloride and hydrogen bromide by nitrogen dioxide. J. Phys. Chem. 64: 602-604.

47. Russe11, J. L., Jr. 1974. Nuclear water splitting and the hydrogen economy. Power Engineering 78(4): 48-51.

48. Schoeppe1, R. J. 1972. Prospects for hydrogen-fueled vehicles. Chem. Tech. 2: 476-480.

49. Sconce, J. S. 1962. Chlorine: Its manufacture, properties and uses. Reinhold Publishing Corporation, New York.

50. Scott, R. B., W. H. Denton, and C. M. Nicholls. 1964. Technology and uses of liquid hydrogen. Macmillan, New York.

51. Shelud'ko, M. K. 1934. Catalytic preparation of hydrochloric acid from chlorine and water. Ukrain. Khem. Zhur. 9: 410-416.

52. Smith, J. M. 1970. Chemical engineering kinetics. 2nd ed. McGraw-Hil1 Book Company, New York.

53. Strickland, G., J. Reilly, and R. H. Wiswall, Jr. 1974. An engineering-scale energy storage reservoir of iron titanium hydride. Conference Proceedings, Hydrogen Energy Conference, Miami, Florida.

54. Wentorf, R. H., Jr., and R. E. Hanneman. 1974. Thermochemica1 hydrogen generation. Science 185(4148): 311-319.

55. Willard, H. H., and H. Dieh1. 1943. Advanced quantitative analysis. D. Van Nost rand Company, Inc., New York.

56. Willson, V. A. 1935. Determination of available chlorine in hyperchlorite solutions by direct titration with sodium thiosulfate. Ind. Eng. Chem., Ana1. Ed. 7: 44-45.

57. Winsche, W. E., K. C. Hoffman, and F. J. Salzano. 1973. Hydrogen: Its future role in the nation's energy economy. Science 180(4013): 1325 . 


\section{ACKNOWLEDGMENT}

The author would like to express deep appreciation to Dr. Dean L. Ulrichson for his guidance and helpful suggestions throughout this study and in preparation of this manuscript.

Gratitude is also due Mr. Mike Murtha for his help in calculating the final results and for his constant consultation. 
APPENDIX I. PARTIAL LISTING OF THERMOCHEMICAL CYCLES WHICH. USE THE REVERSE DEACON REACTION

1. DeBeni, Mark 3 (18)

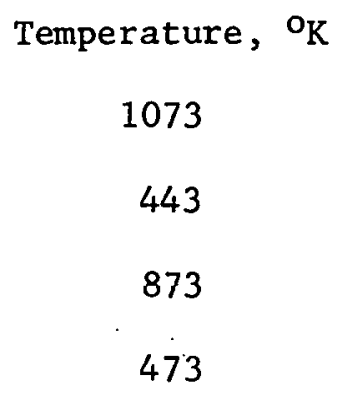

2. Hardy, Mark 4 (18)

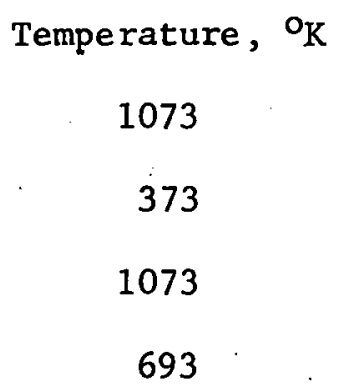

3. Hardy, Mark 7 (18)

Temperature, ${ }^{\circ} \mathrm{K}$

$$
\begin{aligned}
& 3 \mathrm{FeCl}_{2}+4 \mathrm{H}_{2} \mathrm{O} \longrightarrow \mathrm{Fe}_{3} \mathrm{O}_{4}+6 \mathrm{HCl}+\mathrm{H}_{2} \\
& \mathrm{Fe}_{3} \mathrm{O}_{4}+\frac{1}{4} \mathrm{O}_{2} \longrightarrow \frac{3}{2} \mathrm{Fe}_{2} \mathrm{O}_{3} \\
& \frac{3}{2} \mathrm{Fe}_{2} \mathrm{O}_{3}+9 \mathrm{HCl} \longrightarrow 3 \mathrm{FeCl}_{3}+\frac{9}{2} \mathrm{H}_{2} \mathrm{O} \\
& 3 \mathrm{FeCl}_{3} \longrightarrow 3 \mathrm{FeCl}_{2}+\frac{3}{2} \mathrm{Cl}_{2} \\
& \frac{3}{2} \mathrm{H}_{2} \mathrm{O}+\frac{3}{2} \mathrm{Cl}_{2} \longrightarrow 3 \mathrm{HCl}+\frac{3}{2} \mathrm{O}_{2}
\end{aligned}
$$

1073

4. DeBeni, Euratom (18)

$$
\begin{aligned}
& \mathrm{Cl}_{2}+\mathrm{H}_{2} \mathrm{O} \longrightarrow 2 \mathrm{HCl}+\frac{1}{2} \mathrm{O}_{2} \\
& 2 \mathrm{HCl}+2 \mathrm{CrCl}_{2} \longrightarrow 2 \mathrm{CrCl}_{3}+\mathrm{H}_{2}
\end{aligned}
$$




$$
\begin{aligned}
& 2 \mathrm{CrCl}_{3}+2 \mathrm{FeCl}_{2} \longrightarrow 2 \mathrm{CrCl}_{2}+2 \mathrm{FeCl}_{3} \\
& 2 \mathrm{FeCl}_{3}+2 \mathrm{CuCl} \longrightarrow 2 \mathrm{FeCl}_{2}+2 \mathrm{CuCl}_{2} \\
& 2 \mathrm{CuCl}_{2} \longrightarrow 2 \mathrm{CuCl}+\mathrm{Cl}_{2} .
\end{aligned}
$$

5. Funk and Reinstrom, Vanadium Chloride Process (24)

$$
\begin{aligned}
& \mathrm{H}_{2} \mathrm{O}+\mathrm{Cl}_{2} \longrightarrow 2 \mathrm{HCl}+\frac{1}{2} \mathrm{O}_{2} \\
& 2 \mathrm{VCI}_{2}+2 \mathrm{HCl} \longrightarrow 2 \mathrm{VCI}_{3}+\mathrm{H}_{2} \\
& 4 \mathrm{VCl}_{3} \longrightarrow 2 \mathrm{VCl}_{4}+2 \mathrm{VCl}_{2} \\
& 2 \mathrm{VCl}_{4} \longrightarrow 2 \mathrm{VCl}_{3}+\mathrm{Cl}_{2}
\end{aligned}
$$

Temperature, ${ }^{\circ} \mathrm{K}$

6. Knoche, Cremer, and Steinborn, Iron Chloride Process (35)

$$
\begin{gathered}
\text { Temperature, }{ }^{\circ} \mathrm{K} \\
1300 \\
600 \\
700 \\
1300 \\
600
\end{gathered}
$$$$
3 \mathrm{FeCl}_{2}+3 \mathrm{H}_{2} \longrightarrow 6 \mathrm{HCl}+3 \mathrm{Fe}
$$$$
3 \mathrm{Fe}+4 \mathrm{H}_{2} \mathrm{O} \longrightarrow \mathrm{Fe}_{3} \mathrm{O}_{4}+4 \mathrm{H}_{2}
$$$$
\mathrm{H}_{2} \mathrm{O}+\mathrm{Cl}_{2} \longrightarrow 2 \mathrm{HCl}+\frac{1}{2} \mathrm{O}_{2}
$$$$
\frac{1}{2} \mathrm{Cl}_{2}+\mathrm{Fe}_{3} \mathrm{O}_{4}+8 \mathrm{HCl} \longrightarrow \frac{3}{2} \mathrm{Fe}_{2} \mathrm{Cl}_{6}+4 \mathrm{H}_{2} \mathrm{O}
$$$$
\frac{3}{2} \mathrm{Fe}_{2} \mathrm{O}_{6} \longrightarrow 3 \mathrm{FeCl}_{2}+\frac{3}{2} \mathrm{Cl}_{2}
$$

7. Knoche, Chromium Chloride Process (9)

$$
\begin{aligned}
& \mathrm{H}_{2} \mathrm{O}+\mathrm{Cl}_{2} \longrightarrow 2 \mathrm{HCl}+\frac{1}{2} \mathrm{O}_{2} \\
& 2 \mathrm{HCl}+2 \mathrm{CrCl}_{2} \longrightarrow 2 \mathrm{CrCl}_{3}+\mathrm{H}_{2} \\
& 2 \mathrm{CrCl}_{3} \longrightarrow 2 \mathrm{CrCl}_{2}+\mathrm{Cl}_{2}
\end{aligned}
$$

Temperature, ${ }^{\circ} \mathrm{K}$ 973 473 1073

8. Copper Chloride Process (9)

$$
\begin{aligned}
& \mathrm{H}_{2} \mathrm{O}+\mathrm{Cl}_{2} \longrightarrow 2 \mathrm{HCl}+\frac{1}{2} \mathrm{O}_{2} \\
& 2 \mathrm{HCl}+2 \mathrm{CuCl} \longrightarrow 2 \mathrm{CuCl}_{2}+\mathrm{H}_{2} \\
& 2 \mathrm{CuCl}_{2} \longrightarrow 2 \mathrm{CuCl}+\mathrm{Cl}_{2}
\end{aligned}
$$

Temperature, ${ }^{\circ} \mathrm{K}$ 9.73

413

873 
9. HC1 Thermal Decomposition Process (9)

$$
\begin{array}{cc}
\mathrm{H}_{2} \mathrm{O}+\mathrm{Cl}_{2} \longrightarrow 2 \mathrm{HCl}+\frac{1}{2} \mathrm{O}_{2} & \text { Temperature, }{ }_{\mathrm{K}} \\
2 \mathrm{HCl} \longrightarrow \mathrm{H}_{2}+\mathrm{Cl}_{2} & 973
\end{array}
$$




\section{APPENDIX II. EXAMPLE OF CALCULATIONS}

For run Nó. A1:

weight of steam generator before experiment $=1773.1 \mathrm{gm}$ weight of steam generator after experiment $=1741.8 \mathrm{gm}$ operating time for steam valve $=265.5 \mathrm{~min}$. mass flow rate of steam $=\frac{1773.1-1741.8}{265.5}=0.118(\mathrm{gm} / \mathrm{min}$.) volumetric flow rate of steam at $900^{\circ} \mathrm{K}=\frac{0.118}{18} \times 0.082 \times 900=$ 483 (cc/min.)

average oxygen evolution rate at $22^{\circ} \mathrm{C}$ (room temperature):= $4.125 \mathrm{cc} / \mathrm{min}$.

reactor volume $=34 \mathrm{cc}$

volumetric flow rate of chlorine at $900^{\circ} \mathrm{K}=364 \mathrm{cc} / \mathrm{min}$. $\tau=$ space-time $=\frac{34}{364+483}=0.040(\min )=.2.41(\mathrm{sec})$ $\mathrm{X}_{\mathrm{C} 1_{2}}^{\mathrm{f}}=$ conversion based on chlorine $=\frac{4.125 \times 2}{364 \times \frac{295}{900}}=6.91 \%$ assume steam and chlorine are ideal gases at $900^{\circ} \mathrm{K}$ :

$$
\mathrm{C}=\text { concentration }=\frac{\mathrm{n}}{\mathrm{V}}=\frac{\text { No. of moles }}{\text { volume }}=\frac{\mathrm{P}}{\mathrm{RT}}=
$$

\section{pressure}

gas constant $X$ temperature

The total pressure for the system is 1 atm.

$$
\begin{aligned}
\mathrm{P}_{\mathrm{Cl}_{2}}^{\mathrm{O}} & =\text { initial partial pressure of chlorine }=1 \times \frac{364}{364+483} \\
& =0.430(\mathrm{~atm}) \\
\mathrm{P}_{\mathrm{H}_{2} \mathrm{O}}^{\mathrm{O}} & =\text { initial partial pressure of steam }=1 \times \frac{483}{364+483} \\
& =0.570(\mathrm{~atm}) \\
\mathrm{C}_{\mathrm{Ol}_{2}}^{\mathrm{O}} & =\text { initial concentration of chlorine }=\frac{\mathrm{P}_{2}^{\mathrm{O}}}{\mathrm{RT}}=\frac{0.430}{0.082 \times 900} \\
& =0.0058232 \text { (g-moles/l) }
\end{aligned}
$$


$\begin{aligned} & \mathrm{C}_{\mathrm{H}_{2} \mathrm{O}}^{\mathrm{O}}=\text { initial concentration of steam }=\frac{\mathrm{P}_{2}^{\mathrm{O}}}{\mathrm{RT}}=\frac{0.570}{0.082 \times 900} \\ & =0.0072269(\mathrm{~g} \text {-moles } / \mathrm{l})\end{aligned}$
$\mathrm{N}_{1}=$ molal feed ratio of steam to chlorine $=\frac{\mathrm{C}_{\mathrm{H}_{2} \mathrm{O}}^{\mathrm{O}}}{\mathrm{C}_{\mathrm{Cl}_{2}}^{\mathrm{O}}}=\frac{0.0072269}{0.0058232}=1.327$ calculation of equilibrium conversion:

at $900^{\circ} \mathrm{K}$, for $\mathrm{Cl}_{2}+\mathrm{H}_{2} \mathrm{O} \rightleftharpoons 2 \mathrm{HCl}+\frac{1}{2} \mathrm{O}_{2}$

$\mathrm{K}=0.1604=\frac{\left(\mathrm{C}_{\mathrm{HC1}, \text { eq } .}\right)^{2} \cdot\left(\mathrm{C}_{\mathrm{O}_{2}, \text { eq. }}\right)^{1 / 2}}{\left(\mathrm{C}_{\mathrm{Cl}_{2}, \text { eq. }}\right) \cdot\left(\mathrm{C}_{\mathrm{H}_{2} \text { o, eq. }}\right)}$

Using Equations (11), (12), (13), and (14) and rearranging gives

$$
\begin{aligned}
& {\left[2\left(\mathrm{~N}_{1}+1\right)+\mathrm{x}_{\mathrm{Cl} 1_{2}, \text { eq. }}\right]^{1 / 2} \cdot\left(1-\mathrm{x}_{\mathrm{Cl} 1_{2}, \text { eq. }}\right)\left(\mathrm{N}_{1}-\mathrm{X}_{\mathrm{C1} 1_{2}, \text { eq. }}\right)} \\
& \quad=2.903 \mathrm{x}_{\mathrm{C1} 1_{2} \text {, eq. }}^{5 / 2}
\end{aligned}
$$

Then solving by trial and error yields

$$
\mathrm{x}_{\mathrm{Cl}_{2}, \text { eq. }}=57.50 \%
$$


APPENDIX III. SUGGESTED REACTION MECHANISMS FOR

MODELS $G, H$, AND L

1. Mode1 G

The following mechanism is suggested.

$$
\begin{aligned}
& \mathrm{Cl}_{2} \underset{\mathrm{k}_{2}}{\stackrel{\mathrm{k}_{1}}{2}} 2 \mathrm{Cl} \\
& 2 \mathrm{Cl}+\mathrm{H}_{2} \mathrm{O} \frac{\mathrm{k}_{3}}{\mathrm{k}_{4}} \mathrm{HOC} 1+\mathrm{HCl} \\
& \mathrm{HOCl} \stackrel{\mathrm{k}_{5}}{\stackrel{\mathrm{k}_{6}}{=}} \mathrm{HCl}+\frac{1}{2} \mathrm{O}_{2}
\end{aligned}
$$

At steady state:

$$
\begin{aligned}
& \mathrm{k}_{1} \cdot \mathrm{C}_{\mathrm{Cl}_{2}}=\mathrm{k}_{2} \cdot \mathrm{C}_{\mathrm{Cl}}^{2} \cdot \\
& \mathrm{k}_{3} \cdot \mathrm{C}_{\mathrm{Cl} 1}^{2} \cdot \mathrm{C}_{\mathrm{H}_{2} \mathrm{O}}=\mathrm{k}_{4} \cdot \mathrm{C}_{\mathrm{HOCl} 1} \mathrm{C}_{\mathrm{HCl}} \\
& \text { So } \quad \mathrm{C}_{\mathrm{HOCl}}=\frac{\mathrm{k}_{1} \mathrm{k}_{3}}{\mathrm{k}_{2} \mathrm{k}_{4}} \frac{\mathrm{C}_{\mathrm{Cl}_{2}} \mathrm{C}_{\mathrm{H}_{2} \mathrm{O}}}{\mathrm{C}_{\mathrm{HCl}}} .
\end{aligned}
$$

Then rate $=\left(-r_{H O C 1}\right)=k_{5} \cdot C_{H O C 1}-k_{6} \cdot C_{H_{C 1}{ }_{O_{2}}} C^{1 / 2}=\frac{k_{1} k_{3} k_{5}}{k_{2} k_{4}} \frac{{ }_{C_{C 1}} C_{H_{2} O}}{C_{H C 1}}$

$$
-\mathrm{k}_{6} \cdot \mathrm{C}_{\mathrm{HCl}} \mathrm{C}_{\mathrm{O}_{2}}^{1 / 2}
$$

2. Model H

The following mechanisms are suggested.

$$
\begin{aligned}
& \text { a. } 2 \mathrm{Cl}_{2} \frac{\mathrm{k}_{1}}{\mathrm{\textrm {k } _ { 2 }}} 4 \mathrm{Cl} \cdot \\
& 4 \mathrm{Cl} \cdot+2 \mathrm{H}_{2} \mathrm{O} \frac{\mathrm{k}_{3}}{\stackrel{\mathrm{k}_{4}}{=}} 2 \mathrm{HOCl}+2 \mathrm{HCl} \\
& 2 \mathrm{HOC1} \frac{\mathrm{k}_{5}}{\mathrm{k}_{6}} 2 \mathrm{HCl}+\mathrm{O}_{2}
\end{aligned}
$$


At steady state:

$$
\begin{aligned}
& \mathrm{k}_{1} \cdot \mathrm{C}_{\mathrm{Cl}}^{2}=\mathrm{k}_{2} \cdot \mathrm{c}_{\mathrm{Cl}}^{4} \\
& \mathrm{k}_{3} \cdot \mathrm{C}_{\mathrm{C} 1}^{4} \cdot \mathrm{c}_{\mathrm{H}_{2} \mathrm{O}}^{2}=\mathrm{k}_{4} \cdot \mathrm{c}_{\mathrm{HOC} 1}^{2} \cdot \mathrm{C}_{\mathrm{HC} 1}^{2} \\
& \text { So } \quad C_{H O C 1}^{2}=\frac{k_{1} k_{3}}{k_{2} k_{4}} \cdot \frac{C_{C_{2}}^{2} C_{H_{2} O}^{2}}{c_{H C 1}^{2}}
\end{aligned}
$$

Then rate $=\left(-r_{\mathrm{HOCl}}\right)=k_{5} \cdot \mathrm{C}_{\mathrm{HOC1}}^{2}-k_{6} \cdot \mathrm{C}_{\mathrm{HCl} \mathrm{O}_{2}}^{2}=\frac{\mathrm{k}_{1} \mathrm{k}_{3} \mathrm{k}_{5}}{\mathrm{k}_{2} \mathrm{k}_{4}} \frac{\mathrm{C}_{\mathrm{Cl}_{2}}^{2} \mathrm{C}_{\mathrm{H}_{2} \mathrm{O}}^{2}}{\mathrm{c}_{\mathrm{HCl}}^{2}}$

$$
\begin{gathered}
-\mathrm{k}_{6} \cdot \mathrm{C}_{\mathrm{HCl}}^{2} \mathrm{C}_{2} \\
\text { b. } \quad 2 \mathrm{Cl}_{2}+\mathrm{H}_{2} \mathrm{O} \underset{\mathrm{k}_{2}}{\stackrel{\mathrm{k}_{1}}{\rightleftharpoons}} 2 \mathrm{HCl}+\mathrm{Cl}_{2} \mathrm{O} \\
\mathrm{Cl}_{2} \mathrm{O}+\mathrm{H}_{2} \mathrm{O} \underset{\mathrm{k}_{4}}{\stackrel{\mathrm{k}_{3}}{\longrightarrow}} 2 \mathrm{HCl}+\mathrm{O}_{2} \ldots-\ldots-\text { rate controliing step }
\end{gathered}
$$

At steady state:

$$
\begin{aligned}
& \mathrm{k}_{1} \cdot \mathrm{C}_{\mathrm{Cl}_{2}}^{2} \cdot \mathrm{C}_{\mathrm{H}_{2} \mathrm{O}}=\mathrm{k}_{2} \cdot \mathrm{C}_{\mathrm{HCl}}^{2} \cdot \mathrm{C}_{\mathrm{Cl}_{2} \mathrm{O}} \\
& \text { So } \quad \mathrm{C}_{\mathrm{Cl}_{2} \mathrm{O}}=\frac{\mathrm{k}_{1}}{\mathrm{k}_{2}} \cdot \frac{\mathrm{C}_{\mathrm{Cl}_{2}{ }_{2}{ }_{\mathrm{H}_{2} \mathrm{O}}}}{\mathrm{C}_{\mathrm{HC} 1}^{2}} \\
& \text { Then rate }=\left(-\mathrm{r}_{\mathrm{Cl}_{2} \mathrm{O}}\right)=\left(-\mathrm{r}_{\mathrm{H}_{2} \mathrm{O}}\right)=\mathrm{k}_{3} \cdot \mathrm{C}_{\mathrm{Cl}_{2} \mathrm{O}} \cdot \mathrm{C}_{\mathrm{H}_{2} \mathrm{O}}-\mathrm{k}_{4} \mathrm{C}_{\mathrm{HC1}}^{2} \cdot \mathrm{C}_{\mathrm{O}_{2}} \\
& =\frac{k_{1} k_{3}}{k_{2}} \cdot \frac{\mathrm{C}_{\mathrm{Cl}_{2}}^{2} \mathrm{C}_{\mathrm{H}_{2} \mathrm{O}}^{2}}{\mathrm{C}_{\mathrm{HC} 1}^{2}}-\mathrm{k}_{4} \mathrm{C}_{\mathrm{HCl} \mathrm{O}_{2}}^{2}
\end{aligned}
$$

3. Model L

The following chain-reaction mechanisms are suggested. 
74

a.

$$
\begin{aligned}
& \mathrm{Cl}_{2} \frac{\mathrm{k}_{1}}{\mathrm{k}_{2}} 2 \mathrm{Cl} \\
& \mathrm{Cl} \cdot+\mathrm{H}_{2} \mathrm{O} \frac{\mathrm{k}_{3}}{\mathrm{k}_{4}} \mathrm{HCl}+\mathrm{OH} \cdot-\ldots . .-1 .- \text { rate controlling step }
\end{aligned}
$$

At steady state:

$$
\mathrm{k}_{1} \cdot \mathrm{C}_{\mathrm{Cl}_{2}}=\mathrm{k}_{2} \mathrm{c}_{\mathrm{Cl}}^{2} \text {. }
$$

So

$$
\left.\mathrm{C}_{\mathrm{C} 1 .}={ }^{\mathrm{k}} \frac{\mathrm{l}}{\mathrm{k}_{2}}\right)^{1 / 2} \mathrm{C}_{\mathrm{C} 1_{2}}^{1 / 2}
$$

Then

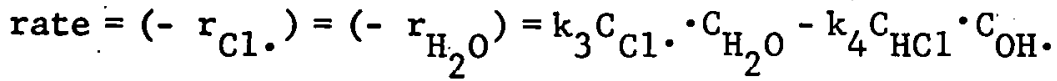

$$
\begin{aligned}
& =\left[\mathrm{k}_{3} \cdot\left(\frac{\mathrm{k}_{1}}{\mathrm{k}_{2}}\right)^{1 / 2}\right] \cdot \mathrm{C}_{\mathrm{Cl}}^{1 / 2} \mathrm{C}_{\mathrm{H}_{2} \mathrm{O}}-\mathrm{k}_{4} \mathrm{C}_{\mathrm{HCl}} \cdot \mathrm{C}_{\mathrm{OH}} \text {. }
\end{aligned}
$$

b.

$$
\begin{aligned}
& \mathrm{Cl}_{2} \frac{\mathrm{k}_{1}}{\stackrel{\mathrm{k}_{2}}{\rightleftharpoons}} 2 \mathrm{Cl} \text {. } \\
& \mathrm{C} 1 \cdot+\mathrm{H}_{2} \mathrm{O} \underset{\frac{\mathrm{k}_{3}}{\mathrm{k}_{4}}}{\mathrm{HOC} 1}+\mathrm{H} \cdot \ldots-\ldots-\ldots-\text { - } \mathrm{Hate} \text { controlling step }
\end{aligned}
$$

At steady state:

$$
\mathrm{k}_{1} \mathrm{C}_{\mathrm{Cl}}=\mathrm{k}_{2} \cdot \mathrm{C}_{\mathrm{C} 1}^{2} \cdot
$$

So

$$
C_{C 1 \cdot}=\left(\frac{k_{1}}{k_{2}}\right)^{1 / 2} \cdot C_{C 1_{2}}^{1 / 2}
$$

Then

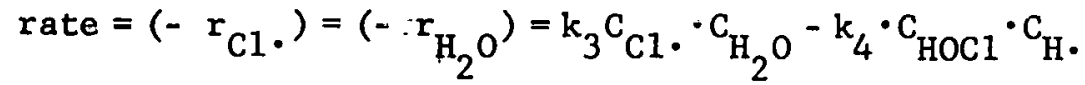

$$
\begin{aligned}
& =\left[k_{3} \cdot\left(\frac{k_{1}}{k_{2}}\right)^{1 / 2}\right] \cdot \mathrm{C}_{\mathrm{Cl}_{2}{ }_{2} \mathrm{Cll}_{2} \mathrm{O}}-\mathrm{k}_{4} \cdot \mathrm{C}_{\mathrm{HOCl}} \cdot \mathrm{C}_{\mathrm{H}} \cdot
\end{aligned}
$$


APPENDIX IV. CALCULATIONS OF FREQUENCY FACTOR AND ACTIVATION ENERGY FOR MODEL B

Arrhenius equation

$k=A e^{-E / R T}$

So

$$
\ell \mathrm{nk}=\operatorname{lnA}-\mathrm{E} / \mathrm{RT}
$$

for model B

$$
\begin{gathered}
k_{1}=0.032 \mathrm{sec}^{-1} \text { at } 900^{\circ} \mathrm{K} \\
k_{1}=0.051 \mathrm{sec}^{-1} \text { at } 950^{\circ} \mathrm{K} \\
\left\{\begin{array}{l}
\ln 0.032=\ln \mathrm{A}-\mathrm{E} / \mathrm{R} \times 900 \\
\ln 0.051=\ln \mathrm{A}-\mathrm{E} / \mathrm{R} \times 950
\end{array}\right.
\end{gathered}
$$

(2)-(1) $\quad \ln \frac{0.051}{0.032}=\frac{50 \mathrm{E}}{\mathrm{R} \times 900 \times 950}$

$$
\begin{aligned}
& E=15.84 \mathrm{Kcal} / \mathrm{g}-\mathrm{mole}=66.26 \cdot \mathrm{KJ} / \mathrm{g}-\mathrm{mole} \\
& A=224 \mathrm{sec}^{-1}
\end{aligned}
$$


APPENDIX V. SUGGESTED REACTION MECHANISMS FOR MODEL B

The following mechanism is suggested to make the forward reaction rate expression fit the expression for model $\mathrm{B}$.

$$
\begin{aligned}
& \mathrm{Cl}_{2} \underset{\mathrm{k}_{2}}{\stackrel{\mathrm{k}_{1}}{2}} 2 \mathrm{Cl} \\
& 2 \mathrm{Cl}+\mathrm{H}_{2} \mathrm{O} \underset{\mathrm{k}_{4}}{\stackrel{\mathrm{k}_{3}}{\rightleftharpoons}} \mathrm{HOCl}+\mathrm{HCl} \\
& \mathrm{HOCl} \underset{\mathrm{k}_{6}}{\stackrel{\mathrm{k}_{5}}{\longrightarrow}} \mathrm{HCl}+\frac{1}{2} \mathrm{O}_{2}
\end{aligned}
$$

At steady state:

$$
\begin{aligned}
& \mathrm{k}_{3} \cdot \mathrm{C}_{\mathrm{Cl}}^{2} \cdot \mathrm{C}_{\mathrm{H}_{2} \mathrm{O}}=\mathrm{k}_{4} \cdot \mathrm{C}_{\mathrm{HOCl}} \cdot \mathrm{C}_{\mathrm{HCl}} \\
& \mathrm{k}_{5} \cdot \mathrm{C}_{\mathrm{HOCl}}=\mathrm{k}_{6} \cdot \mathrm{C}_{\mathrm{HCl}} \cdot \mathrm{C}_{\mathrm{O}_{2}}^{1 / 2} \\
& \mathrm{C}_{\mathrm{Cl}}^{2}=\frac{\mathrm{k}_{4}}{\mathrm{k}_{3}} \cdot \frac{\mathrm{C}_{\mathrm{HOCl}} \cdot \mathrm{C}_{\mathrm{HCl} 1}}{\mathrm{C}_{\mathrm{H}_{2} \mathrm{O}}}=\frac{\mathrm{k}_{4} \mathrm{k}_{6}}{\mathrm{k}_{3} \mathrm{k}_{5}} \cdot \frac{\mathrm{C}_{\mathrm{HCl}}^{2} \mathrm{C}_{2}^{1 / 2}}{\mathrm{C}_{\mathrm{H}_{2} \mathrm{O}}}
\end{aligned}
$$

Then rate $=k_{1} \cdot C_{C_{2}}-k_{2} \cdot C_{C 1}^{2} \cdot=k_{1} \cdot C_{C_{2}}-\frac{k_{2} k_{4} k_{6}}{k_{3} k_{5}} \cdot \frac{C_{H C 1}^{2} C_{2}^{1 / 2}}{C_{\mathrm{H}_{2} O}}$ 
APPENDIX VI. EXAMPLE OF CALCULATIONS OF REYŃOLDS NUMBER

Critical properties of steam and chlorine

steam: $T_{c}=6470 \mathrm{~K}, \mathrm{P}_{\mathrm{c}}=217.7$ atms, $\mu_{\mathrm{c}}=495$ micropoises.

chlorine: $T_{c}=417^{\circ} \mathrm{K}, \mathrm{P}_{c}=76.1$ atms, $\mu_{c}=420$ micropoises

for run No. A-1:

Mole-fraction of steam at the entrance of the reactor is $\frac{483}{847}$.

The following properties are of the reactant-mixture at the

entrance of the reactor.

$$
\begin{aligned}
& \mathrm{P}_{\mathrm{C}}^{*}=\text { critical pressure }=217.7 \times \frac{483}{847}+76.1 \times \frac{364}{847}=156.8 \text { (atms) } \\
& \mathrm{T}_{\mathrm{c}}^{*}=\text { critical temperature }=647 \times \frac{483}{847}+417 \times \frac{364}{847}=548\left({ }^{\circ} \mathrm{K}\right) \\
& \mu_{\mathrm{C}}^{*}=\text { critical viscosity }=495 \times \frac{483}{847}+420 \times \frac{364}{847}=463 \text { (micropoises) } \\
& \mathrm{P}_{\mathrm{r}}^{*}=\text { reduced pressure }=\frac{1}{156.8}=0.0064 \\
& \mathrm{~T}_{\mathrm{r}}^{*}=\text { reduced temperature }=\frac{900}{548}=1.64
\end{aligned}
$$

Then using Figure 175, Hougen's "Chemical Process Principle" P. 871

$$
\begin{aligned}
& \mu_{r}^{*}=\text { reduced viscosity }=0.72 \\
& \mu=\text { viscosity }=\mu_{r}^{*} \times \mu_{c}^{*}=333 \text { (micropoises) } \\
& M=\text { molecular weight }=18 \times \frac{483}{847}+71 \times \frac{364}{847}=40.78 \text { (gms } / g \text {-mole) } \\
& \rho=\text { density }=\frac{P \times M}{R \times T}=\frac{1 \times 40.78}{0.082 \times 900}=0.55 \text { (gms/l) } \\
& \text { Reynolds No. }=\frac{4 \times U^{0} \times \rho}{\pi \times d \times \mu}=\frac{4 \times \frac{847}{60 \times 1000} \times 0.55}{\pi \times \frac{6}{10} \times 3.33 \times 10^{-4}}=.49 .67
\end{aligned}
$$

